\title{
Total Synthesis of Resiniferatoxin Enabled by Photocatalytic Decarboxylative Radical Cyclization
}

\author{
Yuto Hikone, Takehiro Kato, Masanori Nagatomo, and Masayuki Inoue* \\ Graduate School of Pharmaceutical Sciences \\ The University of Tokyo, Hongo, Bunkyo-ku, Tokyo 113-0033, Japan \\ Fax: $(+81) 3-5841-0568$ \\ E-mail: inoue@mol.f.u-tokyo.ac.jp
}

Supporting Information

44 pages

Contents:

page

1. Experimental procedures

S2

2. X-ray structures and crystallographic data of $\mathbf{1 7}$ and $\mathbf{2 2}$

S13

3. Investigation of C8-radical allylation

S16

4. Investigation of photocatalytic decarboxylative radical cyclization

$\mathrm{S} 17$

5. Proposed mechanism of photocatalytic decarboxylative cyclization

$\mathrm{S} 18$

6. Photocatalytic radical cyclization of C1-deoxy analogue S14

S19

7. Computational modeling of $\mathbf{A}$

S20

8. References

$\mathrm{S} 22$

9. NMR spectra

S24 


\section{Experimental procedures}

General method: All reactions sensitive to air or moisture were carried out under argon atmosphere in dry solvents. $\mathrm{CH}_{2} \mathrm{Cl}_{2}$, DMF, $\mathrm{Et}_{2} \mathrm{O}, \mathrm{THF}$, and toluene were purified by Glass Contour solvent dispensing system (Nikko Hansen \& Co., Ltd.). All other reagents were used as supplied unless otherwise noted. All reactions under heating conditions were performed in an oil bath. Photoreactions were carried out in $10 \mathrm{~mL}$ borosilicate glass test tubes, which were placed at $0 \mathrm{~cm}$ from $12 \mathrm{~W}$ blue LED (452 nm, SynLED Parallel Photoreactor, 22900 lx, Sigma-Aldrich) or 23 W blue LED (448 nm, GrassyLeDio RX122 Deep, 194000 lx, volxjapan). Analytical thin-layer chromatography (TLC) was performed using E. Merck Silica gel $60 \mathrm{~F}_{254}$ pre-coated plates $(0.25 \mathrm{~mm})$. Flash column chromatography was performed using 40-50 $\mu \mathrm{m}$ Silica Gel 60N (Kanto Chemical Co., Inc.). Automated flash chromatography was conducted with a Yamazen Smart Flash EPCLC AI-580S system using prepacked silica gel columns. Unless otherwise noted, 40-50 $\mu \mathrm{m}$ Silica Gel $60 \mathrm{~N}$ was used for the purification. Melting points were measured on Yanaco MP-J3 micro melting point apparatus, and were uncorrected. Optical rotations were measured on JASCO P-2200 polarimeter at room temperature using sodium D line. Infrared (IR) spectra were recorded on JASCO FT/IR-4100 spectrometer as a thin film on $\mathrm{KBr} . \quad{ }^{1} \mathrm{H}$ and ${ }^{13} \mathrm{C}\left\{{ }^{1} \mathrm{H}\right\}$ NMR spectra were recorded on JNM-ECS-400, JNM-ECX-500, or JNM-ECZ-500 spectrometer. Chemical shifts were reported in ppm on the $\delta$ scale relative to $\mathrm{CHCl}_{3}$ ( $\delta 7.26$ for $\left.{ }^{1} \mathrm{H} \mathrm{NMR}\right), \mathrm{CDCl}_{3}\left(\delta 77.0\right.$ for $\left.{ }^{13} \mathrm{C}\left\{{ }^{1} \mathrm{H}\right\} \mathrm{NMR}\right), \mathrm{C}_{6} \mathrm{D}_{5} \mathrm{H}\left(\delta 7.16\right.$ for $\left.{ }^{1} \mathrm{H} \mathrm{NMR}\right)$, and $\mathrm{C}_{6} \mathrm{D}_{6}\left(\delta 128.0\right.$ for $\left.{ }^{13} \mathrm{C}\left\{{ }^{1} \mathrm{H}\right\} \mathrm{NMR}\right)$ as internal references. Signal patterns are indicated as $\mathrm{s}$, singlet; d, doublet; t, triplet; q, quartet, m; multiplet, br; broaden peak. The carbon numbering of compounds corresponds to that of resiniferatoxin (1) (Figure S1, Page S2). High-resolution mass spectra were measured on JEOL JMS-T100LP (ESI-TOF/DART) or BRUKER DALTONICS microTOF II (ESI-TOF). X-ray crystallographic analysis was conducted using Inorganic Fine Crystal Structural Diffractometer VariMax Dual (Rigaku Co., Ltd.) with Mo radiation $(\lambda=0.71073 \AA)$.

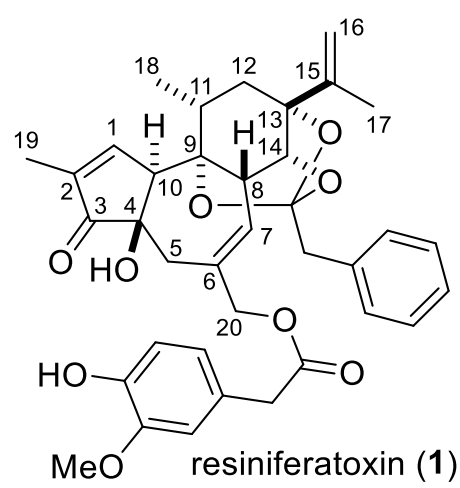

Figure S1. Numbering system of resiniferatoxin (1) 


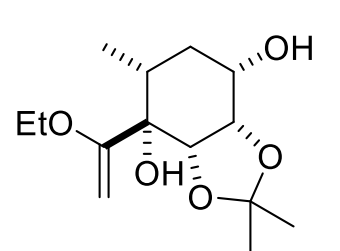

3

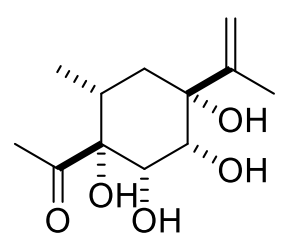

15

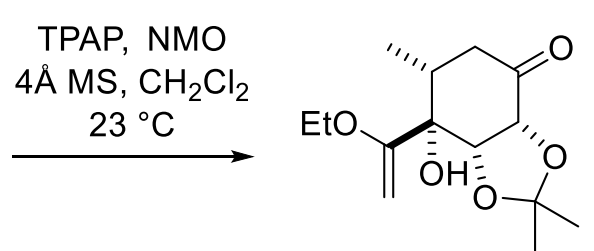

14

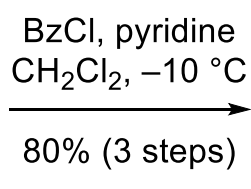

$80 \%$ (3 steps)

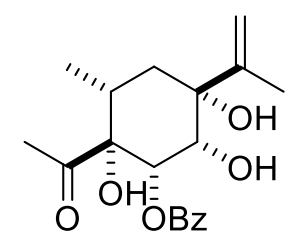

16

Benzoate 16 [CAS: 2139370-83-3]. ${ }^{\mathrm{S} 1}$ Tetrapropylammonium perruthenate (TPAP, $161 \mathrm{mg}$, $0.458 \mathrm{mmol}$ ) was added to a suspension of diol 3 [CAS: $2139370-78-6]^{\mathrm{S} 1}$ (2.63 g, $\left.9.66 \mathrm{mmol}\right)$, $4 \AA \AA$ molecular sieves (4.93 g), and 4-methylmorpholine $N$-oxide (NMO, $1.73 \mathrm{~g}, 14.8 \mathrm{mmol}$ ) in $\mathrm{CH}_{2} \mathrm{Cl}_{2}(48.3 \mathrm{~mL})$ at $0{ }^{\circ} \mathrm{C}$. After being stirred at $23{ }^{\circ} \mathrm{C}$ for $30 \mathrm{~min}$, the reaction mixture was filtered through a pad of silica gel $\left(45.0 \mathrm{~g}, \mathrm{CH}_{2} \mathrm{Cl}_{2} /\right.$ acetone $=1 / 0$ to $\left.15 / 1\right)$. The filtrate was concentrated to afford the crude ketone 14 [CAS: $2139370-79-7]^{\mathrm{S} 1}$ (2.54 g), which was used in the next reaction without further purification. HRMS (ESI-TOF) $\mathrm{m} / \mathrm{z}$ : $[\mathrm{M}+\mathrm{Na}]^{+} \mathrm{Calcd}$ for $\mathrm{C}_{14} \mathrm{H}_{22} \mathrm{O}_{5} \mathrm{Na}$ 293.1359; Found 293.1357.

$\mathrm{LaCl}_{3} \cdot 2 \mathrm{LiCl}(0.6 \mathrm{M}$ THF solution, $48.3 \mathrm{~mL}, 29.0 \mathrm{mmol})$ was added to a solution of the above crude $14(2.54 \mathrm{~g})$ in THF $(10.0 \mathrm{~mL})$ at $0{ }^{\circ} \mathrm{C}$. After the mixture was stirred at $0{ }^{\circ} \mathrm{C}$ for $25 \mathrm{~min}$, isopropenyl magnesium bromide (0.5 M THF solution, $58.0 \mathrm{~mL}, 29.0 \mathrm{mmol})$ was added to the mixture. The reaction mixture was stirred at $0{ }^{\circ} \mathrm{C}$ for $30 \mathrm{~min}$, and then $1 \mathrm{M}$ aqueous $\mathrm{HCl}$ $(58.0 \mathrm{~mL})$ was added to the mixture at $0{ }^{\circ} \mathrm{C}$. After the reaction mixture was stirred at $55^{\circ} \mathrm{C}$ for $16 \mathrm{~h}, 2 \mathrm{M}$ aqueous $\mathrm{NaOH}(16.0 \mathrm{~mL})$ and saturated potassium sodium tartrate $(24 \mathrm{~mL})$ were successively added to the mixture at $0{ }^{\circ} \mathrm{C}$. The resultant mixture was stirred for $30 \mathrm{~min}$, and extracted with EtOAc $(100 \mathrm{~mL} \times 11)$. The combined organic layers were dried over $\mathrm{Na}_{2} \mathrm{SO}_{4}(500 \mathrm{~g})$, filtered, and concentrated. The residue was purified by flash column chromatography on silica gel $\left(75 \mathrm{~g}, \mathrm{CH}_{2} \mathrm{Cl}_{2}\right.$ /acetone $=5 / 1$ to $\left.0 / 1\right)$ to afford the crude tetraol 15 [CAS: $2139370-82-2]^{\mathrm{S} 1}$ (2.32 g), which was used in the next reaction without further purification. To collect the analytical data, 15 was partially purified by flash column chromatography on silica gel $\left(1 \mathrm{~g}, \mathrm{CH}_{2} \mathrm{Cl}_{2}\right.$ /acetone $=1 / 0$ to $\left.3 / 1\right)$.

Tetraol 15: colorless amorphous. ${ }^{1} \mathrm{H}$ NMR $\left(400 \mathrm{MHz}, \mathrm{CDCl}_{3}\right): \delta 5.19(1 \mathrm{H}, \mathrm{d}, J=0.9 \mathrm{~Hz}$, 16a), $5.14(1 \mathrm{H}, \mathrm{s}, \mathrm{H} 16 \mathrm{~b}), 4.59(1 \mathrm{H}, \mathrm{s}, \mathrm{OH}), 4.10(1 \mathrm{H}, \mathrm{ddd}, J=10.0,3.2,0.9 \mathrm{~Hz}, \mathrm{H} 14), 4.03$ $(1 \mathrm{H}, \mathrm{d}, J=10.0 \mathrm{~Hz}, \mathrm{OH}), 3.93(1 \mathrm{H}, \mathrm{dd}, J=11.5,3.2 \mathrm{~Hz}, \mathrm{H} 8), 3.10(1 \mathrm{H}, \mathrm{s}, \mathrm{OH}), 2.68(1 \mathrm{H}, \mathrm{d}, J$ $=11.5 \mathrm{~Hz}, \mathrm{OH}), 2.23\left(3 \mathrm{H}, \mathrm{s}, \mathrm{COCH}_{3}\right), 2.00-1.90(2 \mathrm{H}, \mathrm{m}, \mathrm{H} 11, \mathrm{H} 12 \mathrm{a}), 1.90(3 \mathrm{H}, \mathrm{d}, J=0.9 \mathrm{~Hz}$, H17), $1.67(1 \mathrm{H}, \mathrm{dd}, J=15.0,13.6 \mathrm{~Hz}, \mathrm{H} 12 \mathrm{~b}), 0.74(3 \mathrm{H}, \mathrm{d}, J=6.3 \mathrm{~Hz}, \mathrm{H} 18) .{ }^{13} \mathrm{C}\left\{{ }^{1} \mathrm{H}\right\} \mathrm{NMR}$ 
(100 MHz, $\left.\mathrm{CDCl}_{3}\right): \delta 209.3,144.5,114.6,85.4,75.3,74.0,69.5,34.7,33.2,23.4,19.1,14.0$. HRMS (ESI-TOF) m/z: [M+Na $]^{+}$Calcd for $\mathrm{C}_{12} \mathrm{H}_{20} \mathrm{O}_{5} \mathrm{Na}$ 267.1203; Found 267.1207.

Pyridine (3.14 mL, $38.9 \mathrm{mmol})$ and benzoyl chloride (BzCl, distilled, $1.05 \mathrm{~mL}, 9.04 \mathrm{mmol})$ were successively added to a solution of the above crude tetraol $15(2.32 \mathrm{~g})$ in $\mathrm{CH}_{2} \mathrm{Cl}_{2}(38.9$ $\mathrm{mL})$ at $-10{ }^{\circ} \mathrm{C}$. The reaction mixture was stirred at $-10^{\circ} \mathrm{C}$ for $1 \mathrm{~h}$, and then $\mathrm{BzCl}(60.0 \mu \mathrm{L}$, $0.516 \mathrm{mmol}$ ) was added to the mixture. After the reaction mixture was stirred for $30 \mathrm{~min}$, saturated aqueous $\mathrm{NaHCO}_{3}(15 \mathrm{~mL})$ was added to the mixture at $-10^{\circ} \mathrm{C}$. The resultant mixture was extracted with EtOAc $(30 \mathrm{~mL} \mathrm{x} 4)$. The combined organic layers were dried over $\mathrm{Na}_{2} \mathrm{SO}_{4}(60 \mathrm{~g})$, filtered, and concentrated. The residue was purified by flash column chromatography on silica gel ( $75 \mathrm{~g}$, hexane/EtOAc $=5 / 1$ to $0 / 1)$ to afford benzoate 16 (2.68 g, $7.69 \mathrm{mmol}$ ) in $80 \%$ yield over 3 steps. The analytical data of 16 were identical to those reported previously. ${ }^{\mathrm{S} 1}{ }^{1} \mathrm{H} \mathrm{NMR}\left(500 \mathrm{MHz}, \mathrm{CDCl}_{3}\right): \delta 8.00-7.94(2 \mathrm{H}, \mathrm{m}$, aromatic), $7.53(1 \mathrm{H}$, m, aromatic), 7.45-7.35 (2H, m, aromatic), $5.31(1 \mathrm{H}, \mathrm{s}, \mathrm{H} 16 \mathrm{a}), 5.28(1 \mathrm{H}, \mathrm{d}, J=2.7 \mathrm{~Hz}, \mathrm{H} 8)$, $5.20(1 \mathrm{H}, \mathrm{s}, \mathrm{H} 16 \mathrm{~b}), 4.88(1 \mathrm{H}, \mathrm{br} \mathrm{s}, \mathrm{OH}), 4.66(1 \mathrm{H}, \mathrm{br}, \mathrm{OH}), 4.37(1 \mathrm{H}, \mathrm{d}, J=2.7 \mathrm{~Hz}, \mathrm{H} 14)$, $3.36\left(1 \mathrm{H}\right.$, br s, OH), $2.14\left(3 \mathrm{H}, \mathrm{s}, \mathrm{COCH}_{3}\right), 2.00(1 \mathrm{H}, \mathrm{m}, \mathrm{H} 11), 1.91(3 \mathrm{H}, \mathrm{s}, \mathrm{H} 17), 1.87(1 \mathrm{H}, \mathrm{d}$, $J=14.1 \mathrm{~Hz}, \mathrm{H} 12 \mathrm{a}), 1.75$ (1H, dd, $J=14.1,13.5 \mathrm{~Hz}, \mathrm{H} 12 \mathrm{~b}), 0.75$ (3H, d, $J=6.8 \mathrm{~Hz}, \mathrm{H} 18)$.
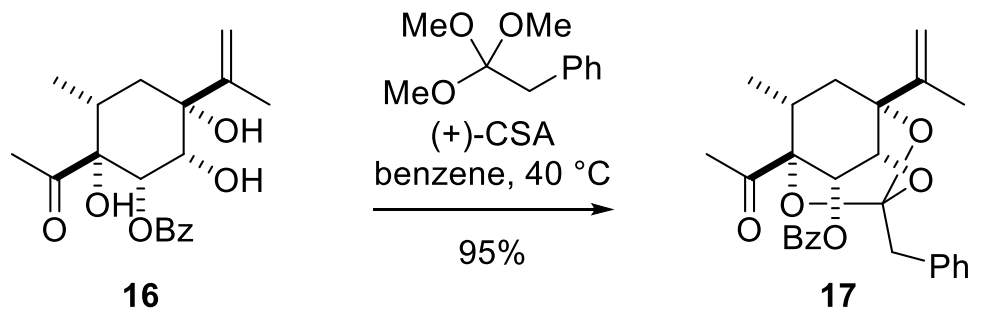

Orthoester 17. (+)-10-Camphorsulfonic acid ((+)-CSA, $289 \mathrm{mg}, 1.24 \mathrm{mmol})$ was added to a solution of benzoate $16(2.83 \mathrm{~g}, 8.12 \mathrm{mmol})$ and trimethyl orthophenylacetate [CAS: $4369-00-0]^{\mathrm{S} 2}(4.50 \mathrm{~mL}, 24.4 \mathrm{mmol})$ in benzene $(40.6 \mathrm{~mL})$ at $23{ }^{\circ} \mathrm{C}$. After the reaction mixture was stirred at $40{ }^{\circ} \mathrm{C}$ for $1.5 \mathrm{~h}$, saturated aqueous $\mathrm{NaHCO}_{3}(20 \mathrm{~mL})$ was added to the mixture at $0{ }^{\circ} \mathrm{C}$. The resultant mixture was extracted with EtOAc $(20 \mathrm{~mL} \times 3)$. The combined organic layers were washed with brine $(16 \mathrm{~mL})$, dried over $\mathrm{Na}_{2} \mathrm{SO}_{4}(60 \mathrm{~g})$, filtered, and concentrated. The residue was purified by flash column chromatography on silica gel (140 g, hexane/Et $2 \mathrm{O}=50 / 1$ to $5 / 1)$ to afford 17 (3.46 g, $7.71 \mathrm{mmol})$ in 95\% yield. The caged C9,13,14-orthophenylacetate structure was confirmed by the X-ray crystallographic analysis (Figure S2, Page S14): colorless hexagonal crystal (recrystallization from hexane/Et ${ }_{2} \mathrm{O}$ ). m.p. 136-138 ${ }^{\circ} \mathrm{C} .[\alpha]_{\mathrm{D}}{ }^{26}+125.8$ (c 1.06, $\mathrm{CHCl}_{3}$ ). IR (film): 3033, 2935, 1724, 1451, 1356, 1270, 1113, 1028, 911, $708 \mathrm{~cm}^{-1}$. ${ }^{1} \mathrm{H}$ NMR (500 MHz, $\left.\mathrm{C}_{6} \mathrm{D}_{6}\right): \delta 8.08-8.03$ (2H, m, aromatic), 7.42-7.35 (2H, m, aromatic), 7.21-7.12 (3H, m, aromatic), $7.09(1 \mathrm{H}, \mathrm{m}$, aromatic), 7.06-6.98 (2H, m, aromatic), $5.14(1 \mathrm{H}, \mathrm{d}, J=3.4 \mathrm{~Hz}, \mathrm{H} 8), 4.81(1 \mathrm{H}, \mathrm{br} \mathrm{s}, \mathrm{H} 16 \mathrm{a}), 4.68(1 \mathrm{H}, \mathrm{s}, \mathrm{H} 16 \mathrm{~b})$, $4.60(1 \mathrm{H}, \mathrm{d}, J=3.4 \mathrm{~Hz}, \mathrm{H} 14), 3.34(1 \mathrm{H}, \mathrm{d}, J=14.2 \mathrm{~Hz}, \mathrm{CCHaHbPh}), 3.30(1 \mathrm{H}, \mathrm{d}, J=14.2$ 
$\mathrm{Hz}, \mathrm{CCHa} H \mathrm{bPh}), 2.48(1 \mathrm{H}, \mathrm{dq}, J=9.1,7.4 \mathrm{~Hz}, \mathrm{H} 11), 2.17\left(3 \mathrm{H}, \mathrm{s}, \mathrm{COCH} H_{3}\right), 1.61(1 \mathrm{H}, \mathrm{dd}, J=$ 14.7, 9.1 Hz, H12a), 1.40 (3H, s, H17), 1.38 (1H, d, $J=14.7$ Hz, H12b), 0.90 (3H, d, $J=7.4$ $\mathrm{Hz}, \mathrm{H} 18) .{ }^{13} \mathrm{C}\left\{{ }^{1} \mathrm{H}\right\}$ NMR $\left(125 \mathrm{MHz}, \mathrm{C}_{6} \mathrm{D}_{6}\right): \delta 209.2,165.5,145.1,135.3,133.2,131.1(2 \mathrm{C})$, 130.2, 130.0 (2C), 128.6 (2C), 128.0 (2C, deduced from HMQC, Page S28), 126.9, 119.0, 111.7, 86.2, 85.8, 77.0, 69.9, 41.2, 35.6, 33.1, 28.4, 18.7, 17.7. HRMS (ESI-TOF) m/z: $[\mathrm{M}+\mathrm{Na}]^{+}$Calcd for $\mathrm{C}_{27} \mathrm{H}_{28} \mathrm{O}_{6} \mathrm{Na} 471.1778$; Found 471.1777 .
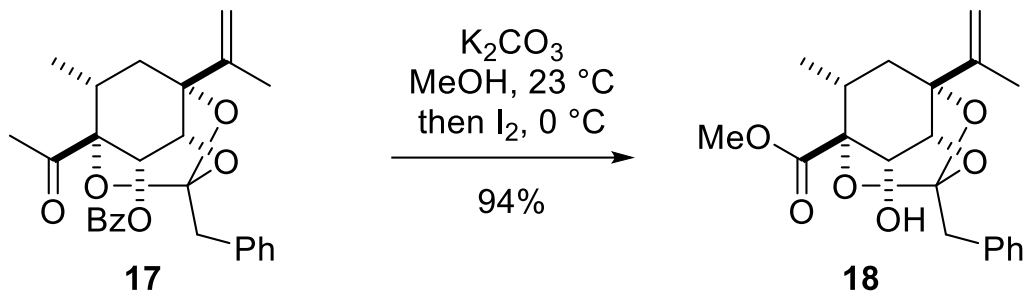

Methyl ester 18. $\quad \mathrm{K}_{2} \mathrm{CO}_{3}(3.71 \mathrm{~g}, 26.9 \mathrm{mmol})$ was added to a solution of orthoester 17 (2.41 $\mathrm{g}, 5.37 \mathrm{mmol})$ in $\mathrm{MeOH}(53.7 \mathrm{~mL})$ at $23{ }^{\circ} \mathrm{C}$. The reaction mixture was stirred at $23{ }^{\circ} \mathrm{C}$ for 1 $\mathrm{h}$, and then $\mathrm{I}_{2}(6.86 \mathrm{~g}, 27.0 \mathrm{mmol})$ was added to the mixture at $0{ }^{\circ} \mathrm{C}$. After the mixture was stirred at $0{ }^{\circ} \mathrm{C}$ for $2 \mathrm{~h}, \mathrm{I}_{2}(1.37 \mathrm{~g}, 5.40 \mathrm{mmol})$ and $\mathrm{K}_{2} \mathrm{CO}_{3}(1.48 \mathrm{~g}, 10.7 \mathrm{mmol})$ were added to the mixture. The reaction mixture was stirred at $0{ }^{\circ} \mathrm{C}$ for $3 \mathrm{~h}$, and then $\mathrm{K}_{2} \mathrm{CO}_{3}(740 \mathrm{mg}, 5.36$ mmol) was added to the mixture. After the reaction mixture was stirred at $0{ }^{\circ} \mathrm{C}$ for $1 \mathrm{~h}$, saturated aqueous $\mathrm{Na}_{2} \mathrm{~S}_{2} \mathrm{O}_{3}(24 \mathrm{~mL})$ and $\mathrm{H}_{2} \mathrm{O}(10 \mathrm{~mL})$ were added to the mixture. After the mixture was concentrated to remove $\mathrm{MeOH}$, the resultant mixture was extracted with EtOAc $(20 \mathrm{~mL} \times 3)$. The combined organic layers were washed with brine $(20 \mathrm{~mL})$, dried over $\mathrm{Na}_{2} \mathrm{SO}_{4}(70 \mathrm{~g})$, filtered, and concentrated. The residue was filtered through a pad of Celite $(5 \mathrm{~g})$ with EtOAc $(100 \mathrm{~mL})$, and the filtrate was concentrated. The residue was purified by flash column chromatography on silica gel (35 g, hexane/EtOAc $=20 / 1$ to $1 / 1)$ to afford methyl ester $18(1.82 \mathrm{~g}, 5.05 \mathrm{mmol})$ in 94\% yield: colorless oil. $[\alpha]_{\mathrm{D}}{ }^{26}+86.5\left(c 1.02, \mathrm{CHCl}_{3}\right)$. IR (film): 3755, 2953, 1756, 1733, 1455, 1436, 1289, 1261, 983, $700 \mathrm{~cm}^{-1}$. ${ }^{1} \mathrm{H}$ NMR (500 $\left.\mathrm{MHz}, \mathrm{C}_{6} \mathrm{D}_{6}\right): \delta$ 7.44-7.39 $(2 \mathrm{H}, \mathrm{m}$, aromatic), 7.18-7.11 $(2 \mathrm{H}, \mathrm{m}$, aromatic), $7.13(1 \mathrm{H}, \mathrm{m}$, aromatic), $4.65(1 \mathrm{H}, \mathrm{s}, \mathrm{H} 16 \mathrm{a}), 4.61(1 \mathrm{H}, \mathrm{m}, \mathrm{H} 16 \mathrm{~b}), 3.91(1 \mathrm{H}, \mathrm{d}, J=3.4 \mathrm{~Hz}, \mathrm{H} 14), 3.48(1 \mathrm{H}$, dd, $J=11.9,3.4 \mathrm{~Hz}, \mathrm{H} 8), 3.46\left(3 \mathrm{H}, \mathrm{s}, \mathrm{CO}_{2} \mathrm{CH}_{3}\right), 3.34(1 \mathrm{H}, \mathrm{d}, J=14.7 \mathrm{~Hz}, \mathrm{CCHaHbPh}), 3.29$ $(1 \mathrm{H}, \mathrm{d}, J=14.7 \mathrm{~Hz}, \mathrm{CCHa} H \mathrm{bPh}), 2.93(1 \mathrm{H}, \mathrm{d}, J=11.9 \mathrm{~Hz}, \mathrm{OH}), 2.45(1 \mathrm{H}, \mathrm{dq}, J=9.1,7.4$ Hz, H11), 1.49 (1H, dd, $J=14.2,9.1 \mathrm{~Hz}, \mathrm{H} 12 \mathrm{a}), 1.37$ (1H, d, $J=14.2 \mathrm{~Hz}, \mathrm{H} 12 \mathrm{~b}), 1.25$ (3H, s, H17), $1.06(3 \mathrm{H}, \mathrm{d}, J=7.4 \mathrm{~Hz}, \mathrm{H} 18) .{ }^{13} \mathrm{C}\left\{{ }^{1} \mathrm{H}\right\} \mathrm{NMR}\left(125 \mathrm{MHz}, \mathrm{C}_{6} \mathrm{D}_{6}\right): \delta 171.2,145.3,134.9$, 131.3 (2C), 128.5 (2C), 127.0, 118.8, 111.5, 85.6, 84.4, 79.3, 67.1, 52.1, 40.9, 34.9, 32.5, 18.6, 17.9. HRMS (ESI-TOF) m/z: [M+Na] $]^{+}$Calcd for $\mathrm{C}_{20} \mathrm{H}_{24} \mathrm{O}_{6} \mathrm{Na} 383.1465$; Found 383.1463. 


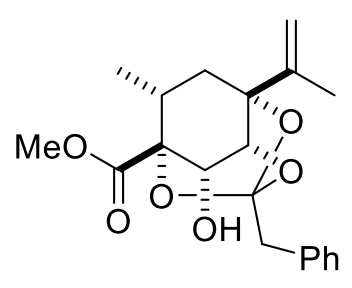

18

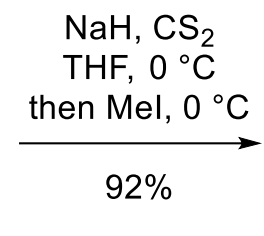

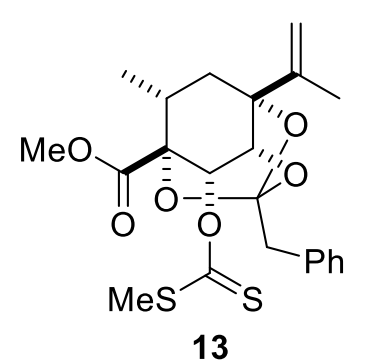

13

Xanthate 13. $\mathrm{CS}_{2}(3.10 \mathrm{~mL}, 51.3 \mathrm{mmol})$ and $\mathrm{NaH}(50-70 \mathrm{wt} \%$ in mineral oil, $1.02 \mathrm{~g}$, 21.3-29.8 mmol) were successively added to a solution of methyl ester 18 (1.82 g, 5.05 $\mathrm{mmol})$ in THF $(50.4 \mathrm{~mL})$ at $0{ }^{\circ} \mathrm{C}$. The reaction mixture was stirred at $0{ }^{\circ} \mathrm{C}$ for $30 \mathrm{~min}$, and then MeI (filtered through a pad of basic $\mathrm{Al}_{2} \mathrm{O}_{3}, 1.58 \mathrm{~mL}, 25.4 \mathrm{mmol}$ ) was added to the mixture. After the reaction mixture was stirred at $0{ }^{\circ} \mathrm{C}$ for $1 \mathrm{~h}$, saturated aqueous $\mathrm{NaHCO}_{3}$ $(20 \mathrm{~mL})$ was added to the mixture. The resultant mixture was extracted with EtOAc $(20 \mathrm{~mL}$ x3). The combined organic layers were washed with brine $(20 \mathrm{~mL})$, dried over $\mathrm{Na}_{2} \mathrm{SO}_{4}(50$ $\mathrm{g}$ ), filtered, and concentrated. The residue was purified by flash column chromatography on silica gel (30 g, hexane/EtOAc $=20 / 1$ to $10 / 1)$ to afford xanthate $13(2.08 \mathrm{~g}, 4.62 \mathrm{mmol})$ in $92 \%$ yield: colorless oil. $[\alpha]_{\mathrm{D}}{ }^{27}+89.8\left(c 0.99, \mathrm{CHCl}_{3}\right)$. IR (film): 2951, 1765, 1734, 1436, 1210, 1178, 1082, 1004, 976, $701 \mathrm{~cm}^{-1} .{ }^{1} \mathrm{H}$ NMR (500 MHz, $\left.\mathrm{C}_{6} \mathrm{D}_{6}\right): \delta 7.43-7.37(2 \mathrm{H}, \mathrm{m}$, aromatic), 7.14-7.09 (2H, m, aromatic), $7.06(1 \mathrm{H}, \mathrm{m}$, aromatic), $5.84(1 \mathrm{H}, \mathrm{d}, J=2.8 \mathrm{~Hz}, \mathrm{H} 8)$, $4.81(1 \mathrm{H}, \mathrm{d}, J=2.8 \mathrm{~Hz}, \mathrm{H} 14), 4.72(1 \mathrm{H}, \mathrm{s}, \mathrm{H} 16 \mathrm{a}), 4.61(1 \mathrm{H}, \mathrm{m}, \mathrm{H} 16 \mathrm{~b}), 3.42\left(3 \mathrm{H}, \mathrm{s}, \mathrm{CO}_{2} \mathrm{CH}_{3}\right)$, $3.41(1 \mathrm{H}, \mathrm{d}, J=14.2 \mathrm{~Hz}, \mathrm{CCHaHbPh}), 3.35(1 \mathrm{H}, \mathrm{d}, J=14.2 \mathrm{~Hz}, \mathrm{CCHa} H \mathrm{bPh}), 2.39(1 \mathrm{H}, \mathrm{dq}, J$ $=9.1,6.8 \mathrm{~Hz}, \mathrm{H} 11), 2.04(3 \mathrm{H}, \mathrm{s}, \mathrm{SCH}), 1.71(1 \mathrm{H}, \mathrm{dd}, J=14.7,9.1 \mathrm{~Hz}, \mathrm{H} 12 \mathrm{a}), 1.41(1 \mathrm{H}, \mathrm{d}, J$ $=14.7 \mathrm{~Hz}, \mathrm{H} 12 \mathrm{~b}), 1.32(3 \mathrm{H}, \mathrm{s}, \mathrm{H} 17), 1.08(3 \mathrm{H}, \mathrm{d}, J=6.8 \mathrm{~Hz}, \mathrm{H} 18) .{ }^{13} \mathrm{C}\left\{{ }^{1} \mathrm{H}\right\} \mathrm{NMR}(125 \mathrm{MHz}$, $\left.\mathrm{C}_{6} \mathrm{D}_{6}\right): \delta 215.6,170.2,144.8,134.8,131.4(2 \mathrm{C}), 128.5$ (2C), 126.9, 119.3, 111.8, 86.0, 81.6, 76.6, 76.0, 52.1, 40.7, 36.3, 32.9, 18.6, 18.5, 17.7. HRMS (ESI-TOF) m/z: $[\mathrm{M}+\mathrm{Na}]^{+}$Calcd for $\mathrm{C}_{22} \mathrm{H}_{26} \mathrm{O}_{6} \mathrm{~S}_{2} \mathrm{Na} 473.1063$; Found 473.1062.

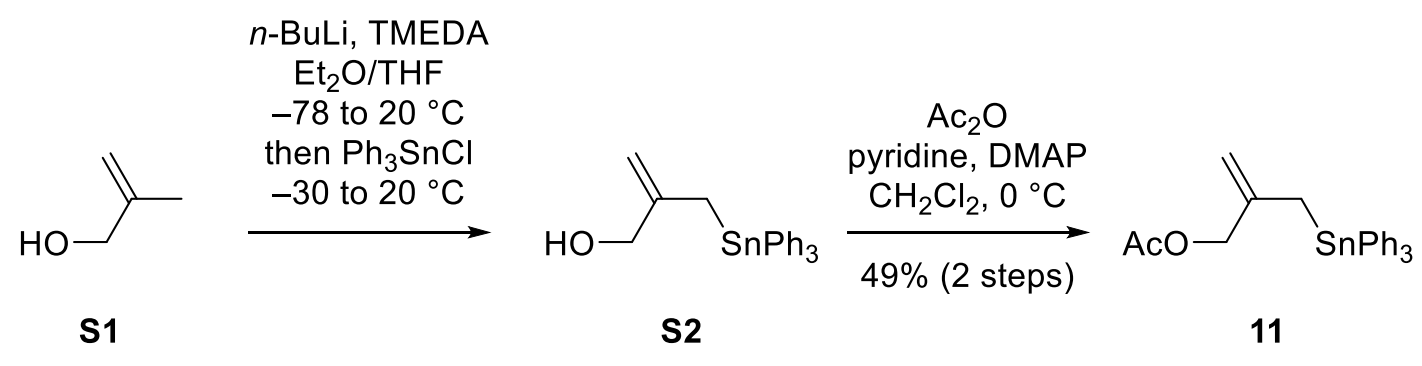

Allyl stannane 11. $\quad N, N, N^{\prime}, N^{\prime}$-Tetramethylethylenediamine (TMEDA, $31.0 \mathrm{~mL}, 207 \mathrm{mmol}$ ) was added to a solution of $n$-BuLi (1.6 M hexane solution, $140 \mathrm{~mL}, 224 \mathrm{mmol})$ in $\mathrm{Et}_{2} \mathrm{O}$ (104 $\mathrm{mL}$ ) at $0{ }^{\circ} \mathrm{C}$, and the mixture was stirred at $0{ }^{\circ} \mathrm{C}$ for $15 \mathrm{~min}$. After the mixture was cooled to $-78^{\circ} \mathrm{C}$, 2-methyl-2-propene-1-ol (S1, $\left.7.00 \mathrm{~mL}, 8.27 \mathrm{mmol}\right)$ was added to the mixture. The reaction mixture was warmed to $0{ }^{\circ} \mathrm{C}$, and then THF $(34.5 \mathrm{~mL})$ was added to the mixture. The reaction mixture was warmed to $20^{\circ} \mathrm{C}$ over $1 \mathrm{~h}$ and stirred for $43 \mathrm{~h}$. After the reaction 
mixture was cooled to $-30{ }^{\circ} \mathrm{C}$, a suspension of $\mathrm{Ph}_{3} \mathrm{SnCl}(35.2 \mathrm{~g}, 91.3 \mathrm{mmol})$ in $\mathrm{THF}(34.5$ $\mathrm{mL}$ ) was added to the mixture. The reaction mixture was warmed to $20^{\circ} \mathrm{C}$ and stirred for 1 h. Then saturated aqueous $\mathrm{NaHCO}_{3}(40 \mathrm{~mL})$ and $\mathrm{H}_{2} \mathrm{O}(40 \mathrm{~mL})$ were added to the mixture at $0{ }^{\circ} \mathrm{C}$. The resultant mixture was extracted with EtOAc $(70 \mathrm{~mL} \times 3)$. The combined organic layers were washed with $\mathrm{H}_{2} \mathrm{O}(70 \mathrm{~mL})$ and brine $(70 \mathrm{~mL})$, dried over $\mathrm{Na}_{2} \mathrm{SO}_{4}(400 \mathrm{~g})$, filtered, and concentrated. The residue was purified by flash column chromatography on silica gel

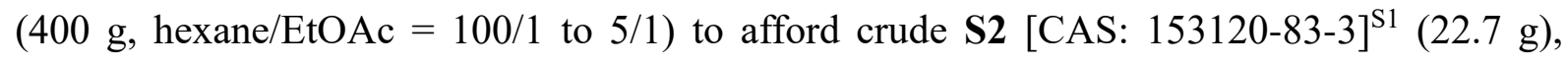
which was used in the next reaction without further purification. HRMS (ESI-TOF) $\mathrm{m} / \mathrm{z}$ : $[\mathrm{M}+\mathrm{Na}]^{+}$Calcd for $\mathrm{C}_{22} \mathrm{H}_{22} \mathrm{OSnNa}$ 445.0585; Found 445.0583.

$\mathrm{Ac}_{2} \mathrm{O}(7.70 \mathrm{~mL}, 81.5 \mathrm{mmol})$ was added to a solution of the above crude $\mathbf{S 2}(22.7 \mathrm{~g})$, 4-dimethylaminopyridine (DMAP, $1.21 \mathrm{~g}, 9.90 \mathrm{mmol}$ ) and pyridine $(17.4 \mathrm{~mL}, 216 \mathrm{mmol})$ in $\mathrm{CH}_{2} \mathrm{Cl}_{2}(72.0 \mathrm{~mL})$ at $0{ }^{\circ} \mathrm{C}$. After the reaction mixture was stirred at $0{ }^{\circ} \mathrm{C}$ for $30 \mathrm{~min}$, saturated aqueous $\mathrm{NaHCO}_{3}(30 \mathrm{~mL})$ was added to the mixture at $0{ }^{\circ} \mathrm{C}$. The resultant mixture was extracted with $\mathrm{CH}_{2} \mathrm{Cl}_{2}(50 \mathrm{~mL} \times 3)$. The combined organic layers were washed with brine $(100 \mathrm{~mL})$, dried over $\mathrm{Na}_{2} \mathrm{SO}_{4}(200 \mathrm{~g})$, filtered, and concentrated. The residue was purified by flash column chromatography on silica gel $(250 \mathrm{~g}$, hexane/EtOAc $=300 / 1$ to $50 / 1)$ to afford ally stannane 11 (18.8 g, $40.6 \mathrm{mmol})$ in 49\% yield over 2 steps: colorless solid. m.p. 46-49 ${ }^{\circ} \mathrm{C}$. IR (film): 3060, 1738, 1424, 1369, 1223, 1070, 1023, 880, 726, $696 \mathrm{~cm}^{-1} .{ }^{1} \mathrm{H}$ NMR $\left(500 \mathrm{MHz}, \mathrm{CDCl}_{3}\right): \delta$ 7.63-7.45 $(6 \mathrm{H}, \mathrm{m}$, aromatic), 7.43-7.32 $(9 \mathrm{H}, \mathrm{m}$, aromatic), $4.91(1 \mathrm{H}, \mathrm{m}$, $\mathrm{CHaHb}=\mathrm{C}), 4.88(1 \mathrm{H}, \mathrm{m}, \mathrm{CHaHb}=\mathrm{C}), 4.42-4.38\left(2 \mathrm{H}, \mathrm{m}, \mathrm{CH}_{2} \mathrm{OAc}\right), 2.48\left(2 \mathrm{H}, \mathrm{s}, J_{\mathrm{H}-\mathrm{Sn}}=69.7\right.$ $\left.\mathrm{Hz}, \mathrm{CH}_{2} \mathrm{SnPh}_{3}\right), 1.79\left(3 \mathrm{H}, \mathrm{s}, \mathrm{OCOCH}_{3}\right) .{ }^{13} \mathrm{C}\left\{{ }^{1} \mathrm{H}\right\} \mathrm{NMR}\left(125 \mathrm{MHz}, \mathrm{CDCl}_{3}\right): \delta 170.6,142.1$, $138.4(3 \mathrm{C}), 136.9\left(6 \mathrm{C}, J_{\mathrm{C}-\mathrm{Sn}}=35.7 \mathrm{~Hz}\right), 129.1(3 \mathrm{C}), 128.6(6 \mathrm{C}), 111.2,68.5,20.6,17.5$. HRMS (ESI-TOF) m/z: [M+Na $]^{+}$Calcd for $\mathrm{C}_{24} \mathrm{H}_{24} \mathrm{O}_{2} \mathrm{SnNa} 487.0691$; Found 487.0684.

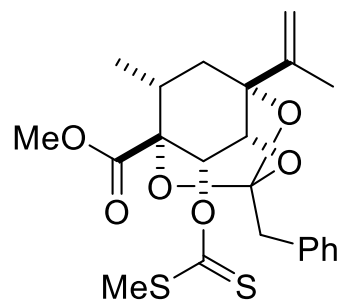

13

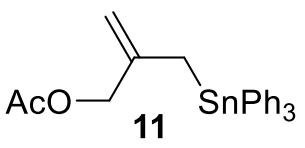

AIBN

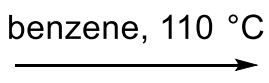

$83 \%$

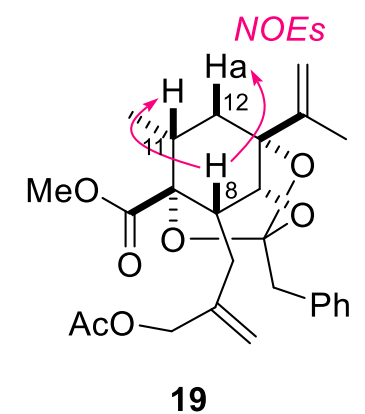

19

Allyl acetate 19. A solution of xanthate $\mathbf{1 3}(1.60 \mathrm{~g}, 3.55 \mathrm{mmol})$, allyl stannane 11 (8.25 g, $17.8 \mathrm{mmol}$ ), and 2,2'-azobis(isobutyronitrile) (AIBN, $146 \mathrm{mg}, 0.889 \mathrm{mmol}$ ) in benzene (17.8 $\mathrm{mL}$ ) was degassed by freeze-thaw procedure (x3). After being stirred at $110{ }^{\circ} \mathrm{C}$ for $3 \mathrm{~h}$, the reaction mixture was cooled to $20^{\circ} \mathrm{C}$ and concentrated. The residue was purified by flash column chromatography (a column consecutively packed with silica gel $110 \mathrm{~g}$ and $10 \%(\mathrm{w} / \mathrm{w})$ $\mathrm{KF}$ contained silica gel $10 \mathrm{~g}$, hexane/EtOAc $=100 / 1$ to $5 / 1)$ to afford allyl acetate $\mathbf{1 9}(1.35 \mathrm{~g}$, 
$2.96 \mathrm{mmol}$ ) in $83 \%$ yield. The C8-stereochemistry of $\mathbf{1 9}$ was determined by the NOE experiment (Page S34): colorless oil. $[\alpha]_{\mathrm{D}}{ }^{29}+53.7$ (c 1.00, $\mathrm{CHCl}_{3}$ ). IR (film): 2950, 1739, 1648, 1449, 1370, 1228, 1091, 1008, 910, $700 \mathrm{~cm}^{-1} .{ }^{1} \mathrm{H}$ NMR (500 MHz, $\left.\mathrm{C}_{6} \mathrm{D}_{6}\right): \delta$ 7.52-7.46 $(2 \mathrm{H}, \mathrm{m}$, aromatic), 7.20-7.12 $(2 \mathrm{H}, \mathrm{m}$, aromatic), $7.07(1 \mathrm{H}, \mathrm{m}$, aromatic), $5.01(1 \mathrm{H}, \mathrm{s}, \mathrm{H} 20 \mathrm{a})$, 5.00 (1H, br s, H20b), 4.84 (1H, br s, H16a), 4.70 (1H, m, H16b), 4.43 (2H, s, H5), 4.35 (1H, d, $J=2.8 \mathrm{~Hz}, \mathrm{H} 14), 3.42(1 \mathrm{H}, \mathrm{d}, J=14.2 \mathrm{~Hz}, \mathrm{CCHaHbPh}), 3.37(1 \mathrm{H}, \mathrm{d}, J=14.2 \mathrm{~Hz}$, $\mathrm{CCHa} H \mathrm{bPh}), 3.35\left(3 \mathrm{H}, \mathrm{s}, \mathrm{CO}_{2} \mathrm{CH}_{3}\right), 2.78(1 \mathrm{H}, \mathrm{dd}, J=13.6,11.9 \mathrm{~Hz}, \mathrm{H} 7 \mathrm{a}), 2.50(1 \mathrm{H}, \mathrm{dq}, J=$ 9.6, 7.4 Hz, H11), 2.23 (1H, dd, $J=13.6,4.0 \mathrm{~Hz}, \mathrm{H} 7 \mathrm{~b}), 1.97$ (1H, ddd, $J=11.9,4.0,2.8 \mathrm{~Hz}$, H8), $1.82(1 \mathrm{H}, \mathrm{dd}, J=14.2,9.6 \mathrm{~Hz}, \mathrm{H} 12 \mathrm{a}), 1.66\left(3 \mathrm{H}, \mathrm{s}, \mathrm{OCOCH}_{3}\right), 1.55(1 \mathrm{H}, \mathrm{d}, J=14.2 \mathrm{~Hz}$, $\mathrm{H} 12 \mathrm{~b}), 1.49$ (3H, s, H17), $1.13(3 \mathrm{H}, \mathrm{d}, J=7.4 \mathrm{~Hz}, \mathrm{H} 18) .{ }^{13} \mathrm{C}\left\{{ }^{1} \mathrm{H}\right\}$ NMR $\left(125 \mathrm{MHz}, \mathrm{C}_{6} \mathrm{D}_{6}\right): \delta$ 172.8, 169.7, 146.4, 141.2, 135.3, 131.4 (2C), 128.0 (2C, deduced from HMQC, Page S34), 126.8, 118.8, 115.1, 111.1, 85.3, 83.1, 77.7, 65.9, 51.7, 41.3, 38.2, 36.8, 33.4, 30.9, 20.3, 19.0, 18.5. HRMS (ESI-TOF) m/z: $[\mathrm{M}+\mathrm{Na}]^{+}$Calcd for $\mathrm{C}_{26} \mathrm{H}_{32} \mathrm{O}_{7} \mathrm{Na}$ 479.2040; Found 479.2040.

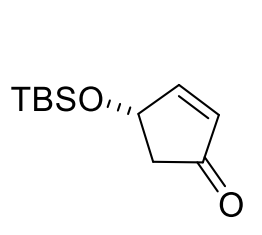

5

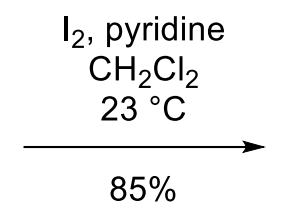

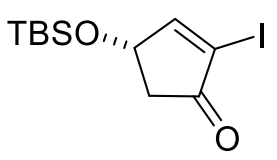

S3

Iodocyclopentenone S3 [CAS: 188308-09-0]. ${ }^{\mathrm{S} 3} \quad \mathrm{I}_{2}(18.3 \mathrm{~g}, 72.1 \mathrm{mmol})$ was added to a solution of cyclopentenone 5 [CAS: $61305-36-0]^{\mathrm{S} 4}(6.95 \mathrm{~g}, 32.7 \mathrm{mmol})$ and pyridine $(26.4$ $\mathrm{mL}, 327 \mathrm{mmol})$ in $\mathrm{CH}_{2} \mathrm{Cl}_{2}(110 \mathrm{~mL})$ at $0{ }^{\circ} \mathrm{C}$. After the reaction mixture was stirred at $23{ }^{\circ} \mathrm{C}$ for $3 \mathrm{~h}$, saturated aqueous $\mathrm{NaHCO}_{3}(20 \mathrm{~mL})$ and saturated aqueous $\mathrm{Na}_{2} \mathrm{~S}_{2} \mathrm{O}_{3}(50 \mathrm{~mL})$ were added to the reaction mixture. The resultant mixture was extracted with $\mathrm{Et}_{2} \mathrm{O}(100 \mathrm{~mL} \times 3)$. The combined organic layers were washed with brine $(200 \mathrm{~mL})$, dried over $\mathrm{Na}_{2} \mathrm{SO}_{4}(200 \mathrm{~g})$, filtered, and concentrated. The residue was purified by flash column chromatography on silica gel $(200 \mathrm{~g}$, hexane/EtOAc $=100 / 1)$ to afford iodocyclopentenone S3 $(9.39 \mathrm{~g}, 27.8$ $\mathrm{mmol})$ in $85 \%$ yield. The analytical data of $\mathbf{S 3}$ were identical to those reported previously. ${ }^{\mathrm{S}}$ ${ }^{1} \mathrm{H}$ NMR (400 MHz, $\left.\mathrm{CDCl}_{3}\right): \delta 7.80(1 \mathrm{H}, \mathrm{dd}, J=2.7,0.91 \mathrm{~Hz}, \mathrm{H} 10), 4.95(1 \mathrm{H}$, ddd, $J=5.9$, 2.7, $1.3 \mathrm{~Hz}, \mathrm{H} 1), 2.87$ (1H, dd, $J=17.2,5.9 \mathrm{~Hz}, \mathrm{H} 2 \mathrm{a}), 2.35(1 \mathrm{H}, \mathrm{ddd}, J=17.2,1.3,0.91 \mathrm{~Hz}$, $\mathrm{H} 2 \mathrm{~b}), 0.91$ (9H, s, $t$-Bu of TBS), 0.14 (3H, s, CH3 of TBS), $0.12\left(3 \mathrm{H}, \mathrm{s}, \mathrm{CH}_{3}\right.$ of TBS).

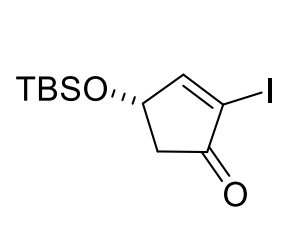

S3

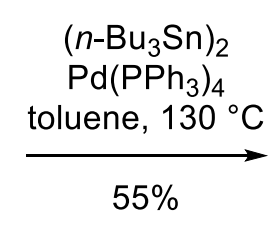

$55 \%$

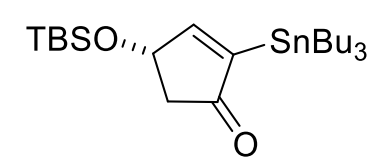

12 
Vinyl stannane 12. A solution of iodocyclopentenone $\mathbf{S 3}$ (9.39 g, $27.8 \mathrm{mmol}), \mathrm{Pd}\left(\mathrm{PPh}_{3}\right)_{4}$ $(1.61 \mathrm{~g}, 1.39 \mathrm{mmol})$, and $\left(n-\mathrm{Bu}_{3} \mathrm{Sn}\right)_{2}(28.0 \mathrm{~mL}, 56.0 \mathrm{mmol})$ in toluene $(139 \mathrm{~mL})$ was degassed by freeze-thaw procedure (x3). After being stirred at $130{ }^{\circ} \mathrm{C}$ for $10.5 \mathrm{~h}$, the reaction mixture was cooled to $0{ }^{\circ} \mathrm{C}$, and $10 \%$ aqueous $\mathrm{KF}(125 \mathrm{~mL})$ was added to the mixture at $0{ }^{\circ} \mathrm{C}$. The resultant mixture was filtered through a pad of Celite $(20 \mathrm{~g})$ with EtOAc $(100 \mathrm{~mL})$. The filtrate was extracted with hexane $(100 \mathrm{~mL} x 2)$. The combined organic layers were washed with saturated aqueous $\mathrm{Na}_{2} \mathrm{~S}_{2} \mathrm{O}_{3}(100 \mathrm{~mL}), 10 \%$ aqueous $\mathrm{KF}(100 \mathrm{~mL})$, and brine $(100 \mathrm{~mL})$, dried over $\mathrm{Na}_{2} \mathrm{SO}_{4}(300 \mathrm{~g})$, filtered, and concentrated. The residue was purified by flash column chromatography (a column consecutively packed with silica gel $500 \mathrm{~g}$ and $10 \%(\mathrm{w} / \mathrm{w})$ $\mathrm{KF}$ contained silica gel $50 \mathrm{~g}$, hexane/EtOAc $=1 / 0$ to $100 / 1)$ to afford vinyl stannane 12 (7.59 g, $15.1 \mathrm{mmol}$ ) in 55\% yield: colorless oil. $[\alpha]_{\mathrm{D}}{ }^{27}-11.0$ (c 0.99, $\left.\mathrm{CHCl}_{3}\right)$. IR (film): 2955, 2928, 2856, 1700, 1254, 1163, 1084, 906, 835, $777 \mathrm{~cm}^{-1} .{ }^{1} \mathrm{H}$ NMR (500 MHz, $\left.\mathrm{C}_{6} \mathrm{D}_{6}\right): \delta 7.52(1 \mathrm{H}, \mathrm{d}$, $\left.J=17.3, J_{\mathrm{H}-\mathrm{Sn}}=27.2 \mathrm{~Hz}, \mathrm{H} 10\right), 4.60(1 \mathrm{H}, \mathrm{ddd}, J=17.3,6.0,2.6 \mathrm{~Hz}, \mathrm{H} 1), 2.45(1 \mathrm{H}, \mathrm{dd}, J=$ $17.9,6.0 \mathrm{~Hz}, \mathrm{H} 2 \mathrm{a}), 2.20(1 \mathrm{H}, \mathrm{dd}, J=17.9,2.6 \mathrm{~Hz}, \mathrm{H} 2 \mathrm{~b}), 1.63\left(6 \mathrm{H}, \mathrm{tt}, J=7.9,7.4, J_{\mathrm{H}-\mathrm{Sn}}=51.0\right.$ $\left.\mathrm{Hz}, \mathrm{SnCH}_{2} \mathrm{CH}_{2} \mathrm{CH}_{2} \mathrm{CH}_{3}\right), 1.37$ (6H, qt, $\left.J=7.4,7.4 \mathrm{~Hz}, \mathrm{SnCH}_{2} \mathrm{CH}_{2} \mathrm{CH}_{2} \mathrm{CH}_{3}\right), 1.14\left(6 \mathrm{H}, \mathrm{t}, J_{\mathrm{H}-\mathrm{Sn}}\right.$ $\left.=53.3, J=7.9 \mathrm{~Hz}, \mathrm{SnCH}_{2} \mathrm{CH}_{2} \mathrm{CH}_{2} \mathrm{CH}_{3}\right), 0.93\left(9 \mathrm{H}, \mathrm{t}, J=7.4 \mathrm{~Hz}, \mathrm{SnCH}_{2} \mathrm{CH}_{2} \mathrm{CH}_{2} \mathrm{CH}_{3}\right), 0.89$ (9H, s, $t$-Bu of TBS), 0.01 (3H, s, $\mathrm{CH}_{3}$ of TBS), -0.04 (3H, s, $\mathrm{CH}_{3}$ of TBS). ${ }^{13} \mathrm{C}\left\{{ }^{1} \mathrm{H}\right\} \mathrm{NMR}$ $\left(125 \mathrm{MHz}, \mathrm{C}_{6} \mathrm{D}_{6}\right): \delta 209.5,172.0,150.1,73.6,45.0,29.5\left(3 \mathrm{C}, J_{\mathrm{C}-\mathrm{Sn}}=21.5 \mathrm{~Hz}\right), 27.6\left(3 \mathrm{C}, J_{\mathrm{C}-\mathrm{Sn}}\right.$ $=57.1 \mathrm{~Hz}), 25.9$ (3C), 18.2, $13.9(3 \mathrm{C}), 9.80$ (3C, $\left.J_{\mathrm{C}-\mathrm{Sn}}=334.8 \mathrm{~Hz}\right),-4.58,-4.68$. HRMS (ESI-TOF) m/z: [M+Na] $]^{+}$Calcd for $\mathrm{C}_{23} \mathrm{H}_{46} \mathrm{O}_{2} \mathrm{SiSnNa}$ 525.2182; Found 525.2201.

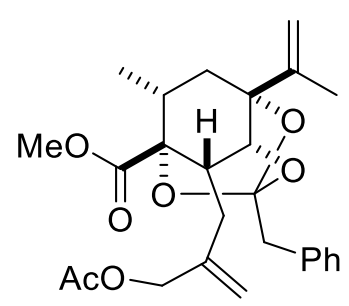

19

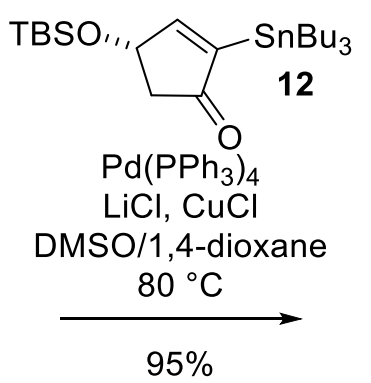

$95 \%$

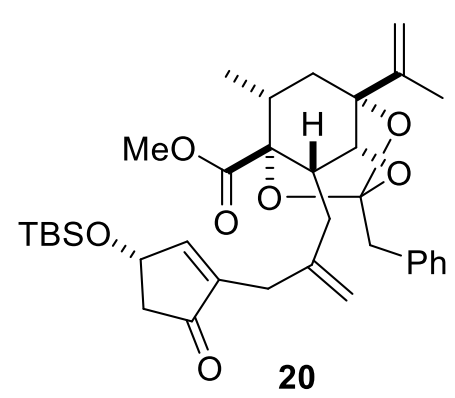

Methyl ester 20. A solution of allyl acetate $19(757 \mathrm{mg}, 1.66 \mathrm{mmol})$ and vinyl stannane 12 (2.98 g, $5.94 \mathrm{mmol})$ in 1,4-dioxane $(8.30 \mathrm{~mL})$ was added to a mixture of $\mathrm{Pd}\left(\mathrm{PPh}_{3}\right)_{4}(290 \mathrm{mg}$, $0.251 \mathrm{mmol}), \mathrm{LiCl}(301 \mathrm{mg}, 7.10 \mathrm{mmol})$, and $\mathrm{CuCl}(583 \mathrm{mg}, 5.89 \mathrm{mmol})$ in DMSO (8.30 $\mathrm{mL})$ at $22{ }^{\circ} \mathrm{C}$ via cannula. The reaction mixture was degassed by freeze-thaw procedure (x3). After the reaction mixture was stirred at $80{ }^{\circ} \mathrm{C}$ for $3 \mathrm{~h}, 10 \%$ aqueous $\mathrm{KF}(16 \mathrm{~mL})$ and saturated aqueous $\mathrm{NH}_{4} \mathrm{Cl}(16 \mathrm{~mL})$ were added to the mixture at $0{ }^{\circ} \mathrm{C}$. The resultant mixture was extracted with $\mathrm{Et}_{2} \mathrm{O}(20 \mathrm{~mL} x 3)$. The combined organic layers were washed with $\mathrm{H}_{2} \mathrm{O}$ (30 mL x3), mixed with Florisil $\left(\mathrm{MgSiO}_{3}, 85 \mathrm{~g}\right)$, filtered through a pad of silica gel (a column consecutively packed with silica gel $40 \mathrm{~g}$ and 10\% (w/w) KF contained silica gel $40 \mathrm{~g}$, EtOAc), and concentrated. The residue was purified by flash column chromatography (a 
column consecutively packed with silica gel $85 \mathrm{~g}$ and $10 \%(\mathrm{w} / \mathrm{w}) \mathrm{KF}$ contained silica gel $37 \mathrm{~g}$, hexane/EtOAc $=70 / 1$ to 3/1) to afford methyl ester 20 (956 mg, $1.57 \mathrm{mmol})$ in $95 \%$ yield: colorless oil. $[\alpha]_{\mathrm{D}}^{22}+29.9$ (c 1.19, $\mathrm{CHCl}_{3}$ ). IR (film): 2952, 2932, 1758, 1714, 1438, 1257 , 1089, 902, 836, $700 \mathrm{~cm}^{-1}$. ${ }^{1} \mathrm{H}$ NMR (500 MHz, $\left.\mathrm{C}_{6} \mathrm{D}_{6}\right): \delta$ 7.53-7.49 (2H, m, aromatic), 7.21-7.15 (2H, m, aromatic), $7.08(1 \mathrm{H}, \mathrm{m}$, aromatic), $6.79(1 \mathrm{H}, \mathrm{m}, \mathrm{H} 10), 4.98(1 \mathrm{H}, \mathrm{s}, \mathrm{H} 20 \mathrm{a})$, $4.94(1 \mathrm{H}$, br s, H16a), 4.81 (1H, br s, H20b), $4.76(1 \mathrm{H}, \mathrm{s}, \mathrm{H} 16 \mathrm{~b}), 4.47(1 \mathrm{H}, \mathrm{d}, J=2.3 \mathrm{~Hz}$, H14), $4.41(1 \mathrm{H}, \mathrm{m}, \mathrm{H} 1), 3.44(1 \mathrm{H}, \mathrm{d}, J=14.2 \mathrm{~Hz}, \mathrm{CCHaHbPh}), 3.40\left(3 \mathrm{H}, \mathrm{s}, \mathrm{CO}_{2} \mathrm{CH}_{3}\right), 3.40$ $(1 \mathrm{H}, \mathrm{d}, J=14.2 \mathrm{~Hz}, \mathrm{CCHaHbPh}), 2.90-2.82(3 \mathrm{H}, \mathrm{m}, \mathrm{H} 5 \mathrm{a}, \mathrm{H} 5 \mathrm{~b}, \mathrm{H} 7 \mathrm{a}), 2.57(1 \mathrm{H}, \mathrm{dq}, J=9.1$, $6.8 \mathrm{~Hz}, \mathrm{H} 11) 2.42(1 \mathrm{H}, \mathrm{dd}, J=17.6,5.7 \mathrm{~Hz}, \mathrm{H} 2 \mathrm{a}), 2.24$ (1H, dd, $J=13.6,4.0 \mathrm{~Hz}, \mathrm{H} 7 \mathrm{~b})$, 2.17-2.10 (2H, m, H2b, H8), 2.00 (1H, dd, $J=14.2,9.1 \mathrm{~Hz}, \mathrm{H} 12 \mathrm{a}), 1.65-1.56$ (4H, m, H12b, H17), $1.15(3 \mathrm{H}, \mathrm{d}, J=6.8 \mathrm{~Hz}, \mathrm{H} 18), 0.89\left(9 \mathrm{H}, \mathrm{s}, t\right.$-Bu of TBS), 0.02 (3H, s, CH $\mathrm{CH}_{3}$ of TBS), -0.05 (3H, s, $\mathrm{CH}_{3}$ of TBS). ${ }^{13} \mathrm{C}\left\{{ }^{1} \mathrm{H}\right\}$ NMR $\left(125 \mathrm{MHz}, \mathrm{C}_{6} \mathrm{D}_{6}\right): \delta 204.0,173.0,158.0,146.4$, 144.5, 142.9, 135.5, 131.4 (2C), 128.0 (2C, deduced from HMQC, Page S38), 126.7, 118.8, 114.5, 111.2, 85.5, 83.3, 77.7, 69.2, 51.7, 45.4, 41.3, 38.0, 37.0, 33.6, 33.5, 30.3, 25.9 (3C), 19.1, 18.6, 18.1, -4.71, -4.73. HRMS (ESI-TOF) $\mathrm{m} / \mathrm{z}$ : $[\mathrm{M}+\mathrm{Na}]^{+}$Calcd for $\mathrm{C}_{35} \mathrm{H}_{48} \mathrm{O}_{7} \mathrm{SiNa}$ 631.3062; Found 631.3070.
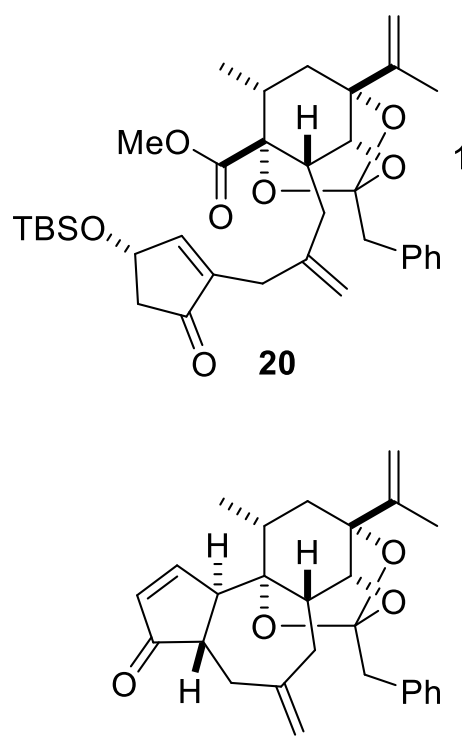

22

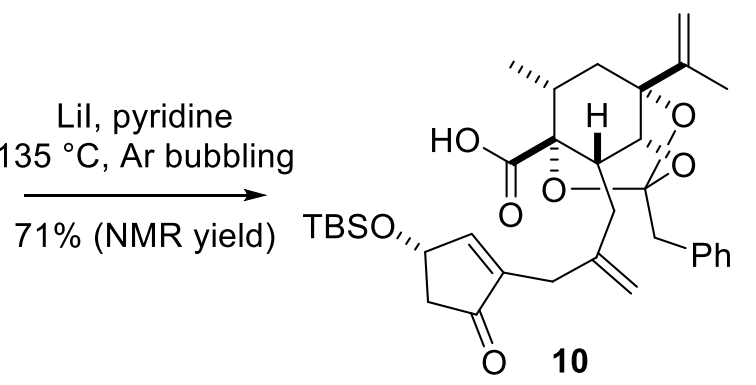

$\operatorname{Ir}($ III) catalyst

(21, $10 \mathrm{~mol} \%)$

$\mathrm{K}_{2} \mathrm{HPO}_{4}$, DMSO blue LED

$35 \%$ (2 steps)

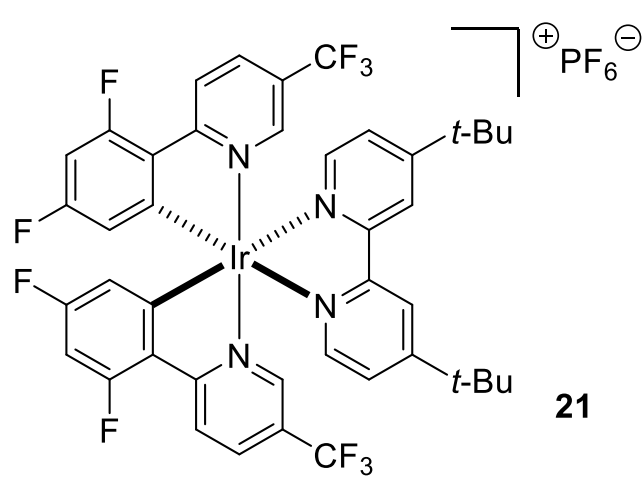

Tricycle 22. A $50 \mathrm{~mL}$ two-necked round-bottom flask with methyl ester 20 (92.7 $\mathrm{mg}, 0.152$ mmol) was equipped with a reflux condenser, which was topped with a three-way cock connected to a bubbler. The apparatus was charged with a solution of LiI (102 $\mathrm{mg}, 0.762$ mmol) in pyridine $(7.60 \mathrm{~mL})$, and the reaction mixture was stirred at $135^{\circ} \mathrm{C}$ for $32 \mathrm{~h}$. Argon was bubbled through the mixture during the reaction. After the reaction mixture was cooled to $0{ }^{\circ} \mathrm{C}$, a solution of $\mathrm{AgNO}_{3}(206 \mathrm{mg}, 1.21 \mathrm{mmol})$ in $\mathrm{H}_{2} \mathrm{O}(3.00 \mathrm{~mL})$ and saturated aqueous $\mathrm{NH}_{4} \mathrm{Cl}(3 \mathrm{~mL})$ were successively added to the mixture. The mixture was filtered through a 
pad of Celite $(5 \mathrm{~g})$ with EtOAc $(50 \mathrm{~mL})$. After the filtrate was extracted with EtOAc $(10 \mathrm{~mL}$ $\mathrm{x} 6$ ), the combined organic layers were concentrated. The residue was filtered through a pad of silica gel $(7.5 \mathrm{~g}, \mathrm{EtOAc} / \mathrm{AcOH}=200 / 1)$. The resultant mixture was concentrated and azeotroped with toluene to afford the crude carboxylic acid $\mathbf{1 0}(95.0 \mathrm{mg})$, which was used in the next reaction without further purification. The yield of $\mathbf{1 0}$ was calculated to be $71 \%$ (0.107 mmol) by ${ }^{1} \mathrm{H}$ NMR analysis using $\mathrm{CH}_{2} \mathrm{Br}_{2}(6.35 \mathrm{mg}, 0.0365 \mathrm{mmol})$ as an internal standard (Page S38). ${ }^{1} \mathrm{H}$ NMR (500 MHz, $\left.\mathrm{C}_{6} \mathrm{D}_{6}\right): \delta$ 7.30-7.20 (2H, m, aromatic), 7.14-7.07 (2 $\mathrm{H}, \mathrm{m}$, aromatic), $7.06(1 \mathrm{H}, \mathrm{m}$, aromatic), $6.77(1 \mathrm{H}, \mathrm{br} \mathrm{s}, \mathrm{H} 10), 4.98(1 \mathrm{H}, \mathrm{s}), 4.96(1 \mathrm{H}, \mathrm{s})$, 4.79-4.76 (2H, m), $4.51(1 \mathrm{H}, \mathrm{d}, J=3.0 \mathrm{~Hz}, \mathrm{H} 14), 4.44$ (1H, m, H1), 3.09 (1H, d, $J=14.4 \mathrm{~Hz}$, $\mathrm{CCHaHbPh}), 3.03(1 \mathrm{H}, \mathrm{d}, J=14.4 \mathrm{~Hz}, \mathrm{CCHa} H \mathrm{bPh}), 2.76(2 \mathrm{H}, \mathrm{s}), 2.61(1 \mathrm{H}, \mathrm{dq}, J=9.1,6.8$ $\mathrm{Hz}, \mathrm{H} 11), 2.56-2.42(2 \mathrm{H}, \mathrm{m}), 2.28(1 \mathrm{H}, \mathrm{m}), 2.16-2.09(2 \mathrm{H}, \mathrm{m}), 1.97(1 \mathrm{H}, \mathrm{dd}, J=14.4,9.1 \mathrm{~Hz}$, H12a), 1.64 (3H, s), 1.51 (1H, d, $J=14.4 \mathrm{~Hz}, \mathrm{H} 12 \mathrm{~b}), 1.00$ (3H, d, $J=6.8 \mathrm{~Hz}, \mathrm{H} 18), 0.89$ (9H, $\mathrm{s}, t$-Bu of TBS), $-0.01\left(3 \mathrm{H}, \mathrm{s}, \mathrm{CH}_{3}\right.$ of TBS), -0.05 (3H, s, CH3 of TBS). HRMS (ESI-TOF) $\mathrm{m} / \mathrm{z}$ : $[\mathrm{M}-\mathrm{H}]^{-}$Calcd for $\mathrm{C}_{34} \mathrm{H}_{45} \mathrm{O}_{7} \mathrm{Si}$ 593.2940; Found 593.2926.

The above crude carboxylic acid $\mathbf{1 0}$ was divided equally into two portions, both of which were placed in the wells of the SynLED Parallel Photoreactor. A solution of the crude 10 (48.8 mg), [4,4'-bis(1,1-dimethylethyl)-2,2'-bipyridine- $N 1, N 1$ '] bis[3,5-difluoro-2-[5-(trifluoro methyl)-2-pyridinyl- $N$ ]phenyl-C]iridium(III) hexafluorophosphate $21(8.77 \mathrm{mg}, 7.82 \mu \mathrm{mol})$, and $\mathrm{K}_{2} \mathrm{HPO}_{4}(68.0 \mathrm{mg}, 0.390 \mathrm{mmol})$ in DMSO $(2.60 \mathrm{~mL})$ was degassed by freeze-thaw procedure (x3). After the reaction mixture was stirred under photoirradiation conditions at $50{ }^{\circ} \mathrm{C}$ for $10 \mathrm{~h}, \mathrm{H}_{2} \mathrm{O}(2 \mathrm{~mL})$ was added to the mixture at $21{ }^{\circ} \mathrm{C}$. The resultant two mixtures were combined and extracted with $\mathrm{Et}_{2} \mathrm{O}(10 \mathrm{~mL} \times 3)$. The combined organic layers were washed with $\mathrm{H}_{2} \mathrm{O}$ (4 mL x3), dried over $\mathrm{Na}_{2} \mathrm{SO}_{4}(20 \mathrm{~g})$, filtered, and concentrated. The residue was purified by automated flash column chromatography using the prepacked silica gel (16 g, hexane/EtOAc $=8 / 1$ to $2 / 1)$ and by flash column chromatography on silica gel ( $5 \mathrm{~g}$, hexane $/ \mathrm{CH}_{2} \mathrm{Cl}_{2}=1 / 1$ to $\left.1 / 20\right)$ to afford tricycle $22(22.5 \mathrm{mg}, 0.0538 \mathrm{mmol})$ in $35 \%$ yield over 2 steps. The C4- and C10-stereochemistries were determined by the X-ray crystallographic analysis (Figure S3, Page S15): colorless plate crystal (recrystallization from hexane/Et $t_{2} \mathrm{O}$ ). m.p. $176-178^{\circ} \mathrm{C} .[\alpha]_{\mathrm{D}}{ }^{30}+45.7$ (c 1.20, $\mathrm{CHCl}_{3}$ ). IR (film): 2938, 2880, 1708, 1451, 1360, 1080, 1035, 990, 901, $700 \mathrm{~cm}^{-1} .{ }^{1} \mathrm{H}$ NMR (500 MHz, $\left.\mathrm{C}_{6} \mathrm{D}_{6}\right): \delta 7.47-7.41$ (2H, m, aromatic), 7.38 $(1 \mathrm{H}, \mathrm{dd}, J=5.7,2.2 \mathrm{~Hz}, \mathrm{H} 1), 7.23-7.18(2 \mathrm{H}, \mathrm{m}$, aromatic $), 7.12(1 \mathrm{H}, \mathrm{m}$, aromatic), $5.85(1 \mathrm{H}$, $\mathrm{dd}, J=5.7,2.3 \mathrm{~Hz}, \mathrm{H} 2), 4.78(1 \mathrm{H}, \mathrm{s}, \mathrm{H} 16 \mathrm{a}), 4.76-4.71$ (2H, m, H16b, H20a), 4.66 (1H, br s, H20b), $3.90(1 \mathrm{H}, \mathrm{d}, J=2.3 \mathrm{~Hz}, \mathrm{H} 14), 3.33(1 \mathrm{H}, \mathrm{d}, J=13.6 \mathrm{~Hz}, \mathrm{CCHaHbPh}), 3.30(1 \mathrm{H}, \mathrm{d}, J=$ $13.6 \mathrm{~Hz}, \mathrm{CCHa} H \mathrm{bPh}), 3.04(1 \mathrm{H}, \mathrm{d}, J=13.6 \mathrm{~Hz}, \mathrm{H} 5 \mathrm{a}), 2.88(1 \mathrm{H}, \mathrm{ddd}, J=4.0,2.3,2.2 \mathrm{~Hz}$, H10), $2.61(1 \mathrm{H}, \mathrm{dd}, J=14.2,10.2 \mathrm{~Hz}, \mathrm{H} 7 \mathrm{a}), 2.08(1 \mathrm{H}, \mathrm{d}, J=14.2 \mathrm{~Hz}, \mathrm{H} 7 \mathrm{~b}), 1.75-1.60$ (2H, m, H4, H5b), 1.50 (1H, dd, $J=14.2,8.5 \mathrm{~Hz}, \mathrm{H} 12 \mathrm{a}), 1.44(3 \mathrm{H}, \mathrm{s}, \mathrm{H} 17), 1.33$ (1H, d, $J=14.2 \mathrm{~Hz}$, H12b), $1.11(1 \mathrm{H}, \mathrm{dq}, J=8.5,6.8 \mathrm{~Hz}, \mathrm{H} 11), 0.96(1 \mathrm{H}, \mathrm{dd}, J=10.2,2.3 \mathrm{~Hz}, \mathrm{H} 8), 0.88$ (3H, d, $J$ $=6.8 \mathrm{~Hz}, \mathrm{H} 18) .{ }^{13} \mathrm{C}\left\{{ }^{1} \mathrm{H}\right\} \mathrm{NMR}\left(125 \mathrm{MHz}, \mathrm{C}_{6} \mathrm{D}_{6}\right): \delta 206.4,162.8,148.1,146.7,135.5,132.4$, 
131.2 (2C), 128.0 (2C, deduced from HMQC, Page S40), 126.9, 118.2, 113.1, 110.9, 84.8, 82.4, 79.7, 57.4, 44.7, 42.9, 41.5, 38.3, 36.6, 35.9, 32.5, 20.6, 18.8. HRMS (ESI-TOF) m/z: $[\mathrm{M}+\mathrm{Na}]^{+}$Calcd for $\mathrm{C}_{27} \mathrm{H}_{30} \mathrm{O}_{4} \mathrm{Na} 441.2036$; Found 441.2027.

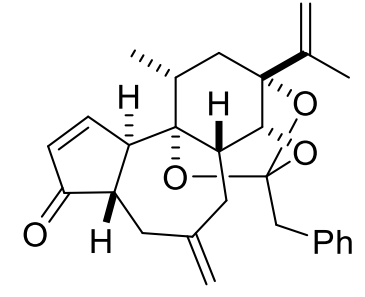

22

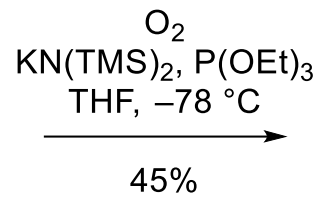

Intermediate 9 [CAS: 2139371-26-7]. ${ }^{\mathrm{S} 1} \quad$ A solution of tricycle $22(38.8 \mathrm{mg}, 0.0927 \mathrm{mmol})$ and $\mathrm{P}(\mathrm{OEt})_{3}(397 \mu \mathrm{L}, 2.32 \mathrm{mmol})$ in THF $(9.27 \mathrm{~mL})$ was degassed by freeze-thaw procedure (x1), and $\mathrm{O}_{2}$ was introduced to the flask. After the mixture was cooled to $-78{ }^{\circ} \mathrm{C}$, potassium bis(trimethylsilyl)amide (KN(TMS)2, $0.5 \mathrm{M}$ toluene solution, $930 \mu \mathrm{L}, 0.465 \mathrm{mmol}$ ) was added to the mixture. The reaction mixture was stirred at $-78{ }^{\circ} \mathrm{C}$ for 30 min under $\mathrm{O}_{2}$ atmosphere (1 atm), and then saturated aqueous $\mathrm{NaHCO}_{3}(2 \mathrm{~mL})$ and $\mathrm{H}_{2} \mathrm{O}(1 \mathrm{~mL})$ were added to the mixture at $-78{ }^{\circ} \mathrm{C}$. The resultant mixture was extracted with EtOAc $(2 \mathrm{~mL} \mathrm{x} 4)$. The combined organic layers were dried over $\mathrm{Na}_{2} \mathrm{SO}_{4}(15 \mathrm{~g})$, filtered, and concentrated. The residue was purified by flash column chromatography on silica gel $(10 \mathrm{~g}$, hexane/EtOAc $=$ $30 / 1$ to $2 / 1)$ to afford intermediate $9(18.3 \mathrm{mg}, 0.0421 \mathrm{mmol})$ in $45 \%$ yield. The analytical data of 9 were identical to those reported previously. ${ }^{\mathrm{S} 1}$ colorless oil. $[\alpha]_{\mathrm{D}}{ }^{23}+63.4(c 0.71$, $\mathrm{MeOH})$. IR (film): 3424, 3070, 3030, 2929, 2880, 1707, 1641, 1577, 1494, $1449 \mathrm{~cm}^{-1} .{ }^{1} \mathrm{H}$ NMR (400 MHz, $\left.\mathrm{C}_{6} \mathrm{D}_{6}\right): \delta 7.52(1 \mathrm{H}, \mathrm{dd}, J=5.9,1.8 \mathrm{~Hz}, \mathrm{H1}), 7.48-7.44$ (2H, m, aromatic), 7.25-7.18 (2H, m, aromatic), $7.12(1 \mathrm{H}, \mathrm{m}$, aromatic), $5.80(1 \mathrm{H}, \mathrm{dd}, J=5.9,3.2 \mathrm{~Hz}, \mathrm{H} 2), 4.84$ (1H, br s, H20a), 4.79 (1H, s, H16a), 4.75 (1H, s, H20b), 4.72 (1H, s, H16b), 3.95 (1H, d, J= $2.3 \mathrm{~Hz}, \mathrm{H} 14), 3.35(1 \mathrm{H}, \mathrm{d}, J=14.1 \mathrm{~Hz}, \mathrm{CCHaHbPh}), 3.31(1 \mathrm{H}, \mathrm{d}, J=14.1 \mathrm{~Hz}, \mathrm{CCHaHbPh})$, 3.02 (1H, m, H10), 2.69-2.58 (2H, m, H5a, H7a), 2.50 (1H, dq, $J=9.1,7.2$ Hz, H11), 2.23 $(1 \mathrm{H}, \mathrm{d}, J=14.1 \mathrm{~Hz}, \mathrm{H} 7 \mathrm{~b}), 2.02(1 \mathrm{H}, \mathrm{s}, \mathrm{OH}), 1.89(1 \mathrm{H}, \mathrm{dd}, J=10.4,2.3 \mathrm{~Hz}, \mathrm{H} 8), 1.82(1 \mathrm{H}, \mathrm{dd}$, $J=14.1,9.1 \mathrm{~Hz}, \mathrm{H} 12 \mathrm{a}), 1.67$ (1H, d, $J=15.0 \mathrm{~Hz}, \mathrm{H} 5 \mathrm{~b}), 1.43$ (3H, s, H17), 1.39 (1H, d, $J=$ $14.1 \mathrm{~Hz}, \mathrm{H} 12 \mathrm{~b}), 1.01(3 \mathrm{H}, \mathrm{d}, J=7.2 \mathrm{~Hz}, \mathrm{H} 18) .{ }^{13} \mathrm{C}\left\{{ }^{1} \mathrm{H}\right\} \mathrm{NMR}\left(100 \mathrm{MHz}, \mathrm{C}_{6} \mathrm{D}_{6}\right): \delta 207.2$, 164.2, 146.9, 145.7, 135.7, 131.2 (2C), 129.2, 128.0 (2C, deduced from HMQC, Page S42), 126.9, 118.1, 116.3, 110.8, 85.1, 82.4, 80.4, 74.0, 57.6, 45.0, 41.9, 41.7, 37.2, 35.9, 33.9, 20.3, 18.9. HRMS (ESI-TOF) m/z: [M+Na] $]^{+}$Calcd for $\mathrm{C}_{27} \mathrm{H}_{30} \mathrm{O}_{5} \mathrm{Na} 457.1986$; Found 457.1982. 


\section{X-ray structures and crystallographic data of 17 and 22}

Table S1. Crystal data and structure refinement for $\mathbf{1 7}$ and $\mathbf{2 2}$

\begin{tabular}{|c|c|c|}
\hline Compound & 17 & 22 \\
\hline CCDC number & 2112687 & 2112688 \\
\hline Molecular formula & $\mathrm{C}_{27} \mathrm{H}_{28} \mathrm{O}_{6}$ & $\mathrm{C}_{27} \mathrm{H}_{30} \mathrm{O}_{4}$ \\
\hline Formula weight & 448.49 & 418.51 \\
\hline Temperature $(\mathrm{K})$ & 93 & 93 \\
\hline Wavelength $(\AA)$ & 0.71073 & 0.71073 \\
\hline Crystal color, habit & colorless, hexagonal & colorless, plate \\
\hline Crystal size $\left(\mathrm{mm}^{3}\right)$ & $0.30 \times 0.20 \times 0.20$ & $0.20 \times 0.05 \times 0.02$ \\
\hline Crystal system & monoclinic & orthorhombic \\
\hline Space group & $\mathrm{P} 12_{1} 1(\# 4)$ & $\mathrm{P} 2{ }_{1} 2_{1} 2_{1}(\# 19)$ \\
\hline Unit cell dimensions & & \\
\hline $\mathrm{a}(\AA)$ & $10.2209(3)$ & $9.3705(3)$ \\
\hline $\mathrm{b}(\AA)$ & $10.8235(3)$ & $14.5501(4)$ \\
\hline c $(\AA)$ & $10.3216(3)$ & $15.5834(5)$ \\
\hline$\alpha\left(^{\circ}\right)$ & 90 & 90 \\
\hline$\beta\left(^{\circ}\right)$ & $94.651(3)$ & 90 \\
\hline$\gamma\left(\left(^{\circ}\right)\right.$ & 90 & 90 \\
\hline Volume $\left(\AA^{3}\right)$ & $1138.08(6)$ & $2124.67(11)$ \\
\hline$Z$ & 2 & 4 \\
\hline Density (calculated) $\left(\mathrm{g} / \mathrm{cm}^{3}\right)$ & 1.309 & 1.308 \\
\hline$\mu(\operatorname{MoK} \alpha)\left(\mathrm{cm}^{-1}\right)$ & 0.92 & 0.86 \\
\hline$F(000)$ & 476 & 896 \\
\hline Index ranges & $-13<=\mathrm{h}<11$ & $-11<=\mathrm{h}<12$ \\
\hline & $-14<=\mathrm{k}<14$ & $-13<=\mathrm{k}<19$ \\
\hline & $-13<=1<13$ & $-19<=1<20$ \\
\hline Reflections collected & 14661 & 14458 \\
\hline Independent reflections & 5266 & 4947 \\
\hline $\mathrm{R}$ (int) & 0.0324 & 0.0329 \\
\hline Completeness to theta & $25.242^{\circ}, 100.00 \%$ & $25.242^{\circ}, 100.00 \%$ \\
\hline Max. and min. transmission & $1.000,0.42531$ & $1.000,0.41646$ \\
\hline Refinement method & \multicolumn{2}{|c|}{ Full-matrix least-squares on $\mathrm{F}^{2}$} \\
\hline $\begin{array}{l}\text { No. Observations } \\
\text { (All reflections) }\end{array}$ & 5266 & 4947 \\
\hline No. Variables & 309 & 295 \\
\hline Reflection/Parameter Ratio & 17.04 & 16.77 \\
\hline Goodness-of-fit on $\mathrm{F}^{2}$ & 1.117 & 1.062 \\
\hline Residuals: R1 $(I>2.00 \sigma(I))$ & 0.0337 & 0.0354 \\
\hline $\begin{array}{l}\text { Residuals: } \mathrm{R} \\
\text { (All reflections) }\end{array}$ & 0.0365 & 0.0391 \\
\hline $\begin{array}{l}\text { Residuals: wR2 } \\
\text { (All reflections) }\end{array}$ & 0.0958 & 0.0843 \\
\hline $\begin{array}{l}\text { Max. and min. peak in Final } \\
\text { Diff. Map }\left(\mathrm{e}-/ \AA^{3}\right)\end{array}$ & $0.282,-0.350$ & $0.203,-0.251$ \\
\hline
\end{tabular}




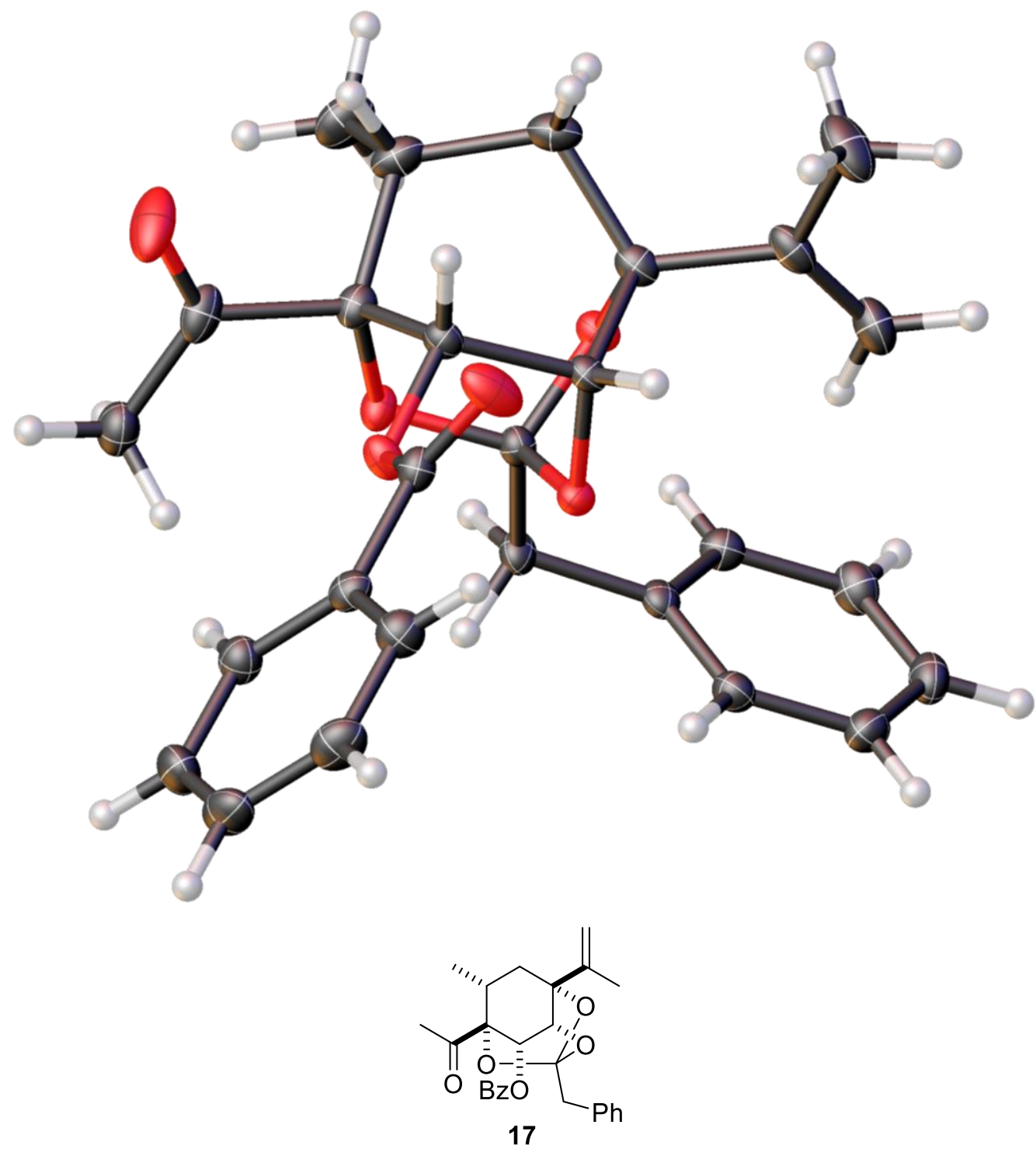

Figure S2. X-ray crystallographic structure of 17 (CCDC 2112687) with thermal ellipsoids at the $50 \%$ probability level (ORTEP) 


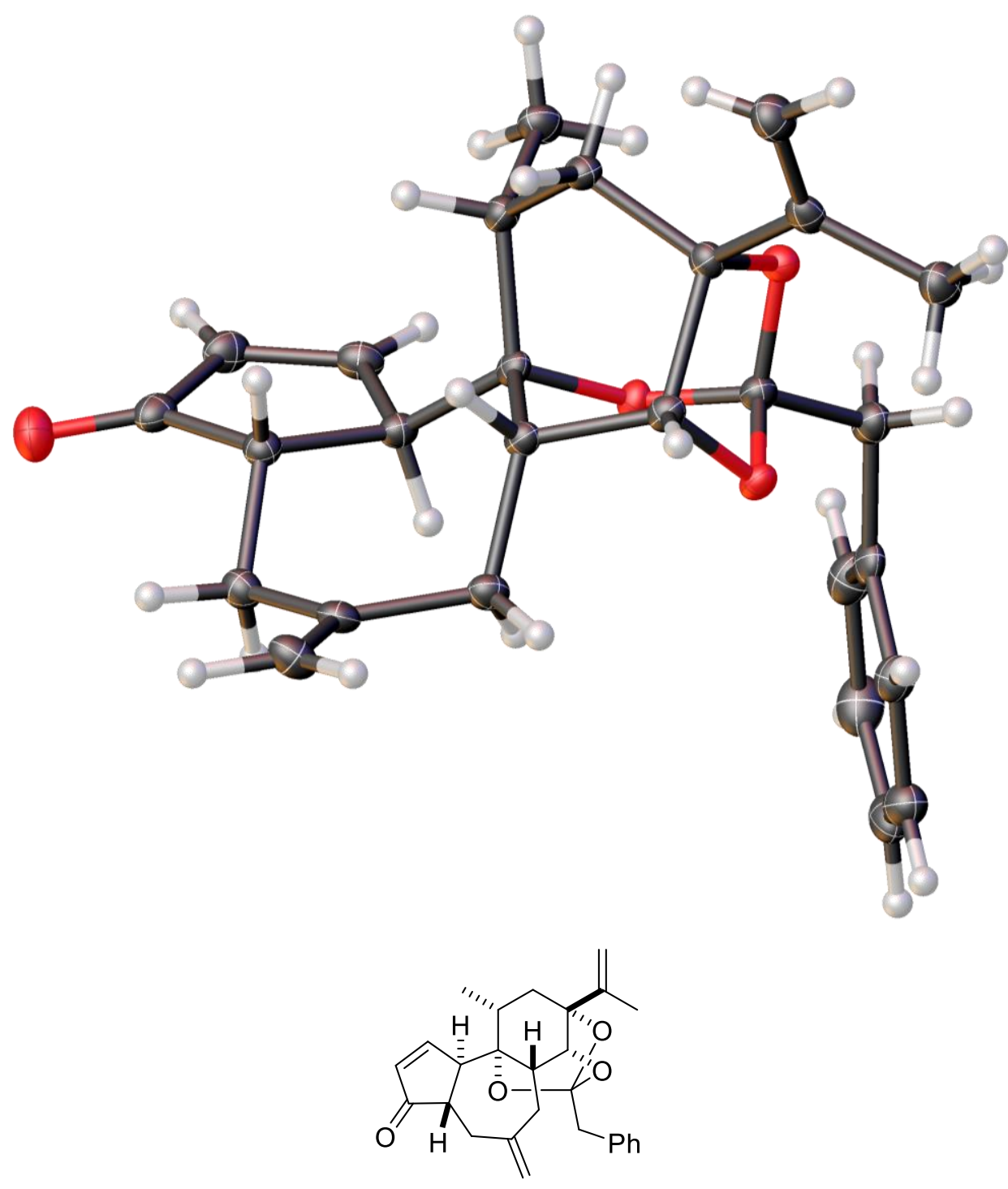

22

Figure S3. X-ray crystallographic structure of 22 (CCDC 2112688) with thermal ellipsoids at the $50 \%$ probability level (ORTEP) 


\section{Investigation of C8-radical allylation}

To evaluate the effect of the C9-substituent, orthoacetates $\mathbf{S 4}{ }^{\mathrm{S} 5}\left(\mathrm{R}^{1}=\mathrm{C}(\mathrm{OEt})=\mathrm{CH}_{2}\right)$ and $\mathbf{S 5}^{\mathrm{S} 6}$ $\left(\mathrm{R}^{1}=\mathrm{CO}_{2} \mathrm{Me}\right)$ were selected as model substrates. When xanthate $\mathbf{S 4}$ was subjected to 2-((triphenylstannyl)methyl)allyl acetate (11) and $\mathrm{AIBN}$ in $\mathrm{PhCl}$ at $110{ }^{\circ} \mathrm{C}$ (entry 1), no carbon-extended product $\mathbf{S 6}$ was observed. In contrast, treatment of S5 with $\mathbf{1 1}$ and AIBN in refluxing benzene gave rise to the adduct $\mathbf{S} 7$ in 70\% NMR yield (entry 2). Thus, the less bulky methoxycarbonyl group of $\mathbf{S 5}$ was found to be more favorable for the intermolecular radical reaction than the ethoxyvinyl counterpart of S4. ${ }^{\mathrm{S} 7}$ The yield of $\mathbf{S 7}$ was also highly dependent on the $\mathrm{R}^{2}$ group of allyl stannane (entries $\left.2-4\right)$. Changing $11\left(R^{2}=P h\right.$, entry 2$)$ to S8 $\left(\mathrm{R}^{2}=n\right.$-Bu, entry 3$)$ or $\mathbf{S 9}\left(\mathrm{R}^{2}=\mathrm{Me}\right.$, entry 4$)$ decreased the yield, confirming that 11 was the most effective allylation reagent among the three.

Table S2. Investigation of C8-radical allylation ${ }^{a}$

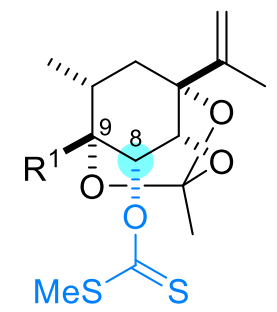

S4: $\mathrm{R}^{1}=\mathrm{C}(\mathrm{OEt})=\mathrm{CH}_{2}$

S5: $\mathrm{R}^{1}=\mathrm{CO}_{2} \mathrm{Me}$

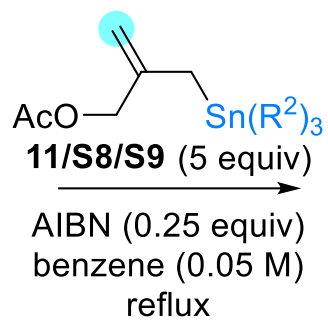

reflux

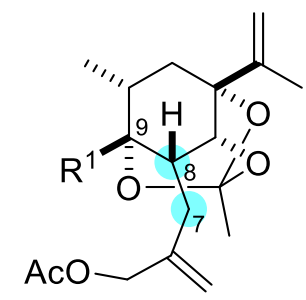

S6: $\mathrm{R}^{1}=\mathrm{C}(\mathrm{OEt})=\mathrm{CH}_{2}$ S7: $\mathrm{R}^{1}=\mathrm{CO}_{2} \mathrm{Me}$

$\begin{array}{lllll}\text { entry } & \text { xanthate } & \mathrm{R}^{1} & \mathrm{R}^{2} & \text { result }^{b} \\ 1^{c} & \mathbf{S 4} & \mathrm{C}(\mathrm{OEt})=\mathrm{CH}_{2} & \mathrm{Ph}(\mathbf{1 1}) & \mathbf{S 6 :} 0 \% \\ 2 & \mathbf{S 5} & \mathrm{CO}_{2} \mathrm{Me} & \mathrm{Ph}(\mathbf{1 1}) & \mathbf{S 7}: 70 \% \\ 3 & \mathbf{S 5} & \mathrm{CO}_{2} \mathrm{Me} & n-\mathrm{Bu}(\mathbf{S 8}) & \mathbf{S 7}: 61 \% \\ 4 & \mathbf{S 5} & \mathrm{CO}_{2} \mathrm{Me} & \mathrm{Me}(\mathbf{S 9}) & \mathbf{S 7}: 52 \%\end{array}$

${ }^{a}$ Reaction conditions: The reactions were conducted using 41-55 $\mu$ mol of S5 with 11/S8/S9 (5 equiv) and AIBN (0.25 equiv) in refluxing benzene $(0.05 \mathrm{M}) .{ }^{b}$ Yields were determined by ${ }^{1} \mathrm{H}$ NMR analysis using $p$-xylene as the internal standard. ${ }^{c}$ The reaction was conducted using $25 \mu \mathrm{mol}$ of $\mathbf{S 4}$ with 11 (10 equiv), $\operatorname{AIBN}\left(0.5\right.$ equiv) in $\mathrm{PhCl}(0.05 \mathrm{M})$ at $110^{\circ} \mathrm{C}$. 


\section{Investigation of photocatalytic decarboxylative radical cyclization}

To expedite the reaction optimization, orthoacetate $\mathbf{S 1 0},{ }^{\mathrm{S} 6}$ which only differs in the orthoester structure of 10, was used as the model substrate. Irradiation of S10 with blue LED (452 nm) in the presence of iridium(III) complex 21 (10 mol\%) and $\mathrm{K}_{2} \mathrm{HPO}_{4}$ (1.5 equiv) in DMF at $50{ }^{\circ} \mathrm{C}$ afforded the seven-membered ring S11 in 32\% NMR yield (entry 1). Analysis of ESI mass spectra revealed that DMF participated in this reaction through formation of the corresponding acyl radical, thereby lowering the yield of S11. Exchanging DMF with DMSO indeed suppressed the generation of the solvent-derived byproducts, furnishing S11 (20\%) along with $\alpha, \beta$-unsaturated ketone S12 (21\%) arising from the base-induced $\beta$-elimination of the C1-OTBS group (entry 2). Upon doubling the amount of $\mathrm{K}_{2} \mathrm{HPO}_{4}(3.0$ equiv), the cyclized products converged into enone S12 in 57\% yield (entry 3). When the catalyst was changed from 21 to the organo-photocatalyst $4 \mathrm{CzIPN},{ }^{\mathrm{S} 8, \mathrm{~S} 9} \mathbf{S 1 2}$ was obtained via concomitant $\beta$-elimination, but only in moderate yield (31\%, entry 4$)$.

Table S3. Investigation of photocatalytic decarboxylative radical cyclization ${ }^{a}$
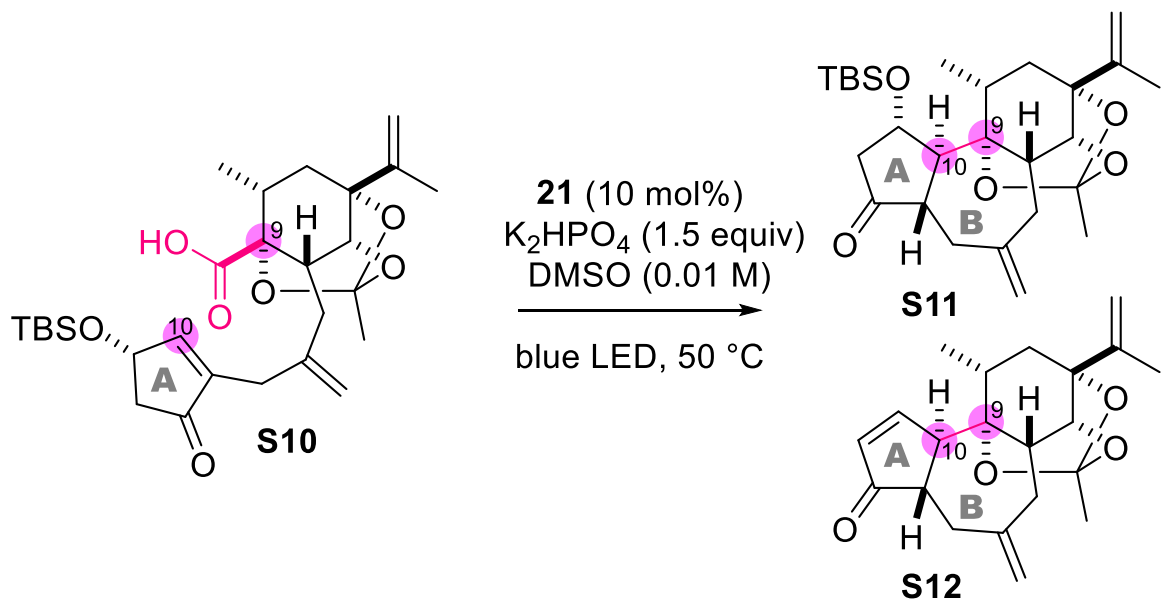

$\begin{array}{lll}\text { entry } & \text { deviation from entry } 2 & \text { result }^{b} \\ 1 & \text { DMF instead of DMSO } & \text { S11: } 32 \% \\ 2 & \text { standard conditions } & \text { S11: } 20 \%, \text { S12: } 21 \% \\ 3 & \mathrm{~K}_{2} \mathrm{HPO}_{4} \text { (3.0 equiv) } & \mathbf{S 1 2 :} 57 \% \\ 4 & 4 \mathrm{CzIPN} \text { instead of 21 } & \mathbf{S 1 2 :} 31 \%\end{array}$

${ }^{a}$ Reaction conditions: The reactions were conducted using $10-15 \mathrm{mg}$ of S10 with photocatalyst $(10 \mathrm{~mol} \%)$ and base $\left(1.5\right.$ equiv) in solvent $(0.01 \mathrm{M})$ at $50{ }^{\circ} \mathrm{C} .{ }^{b}$ Yields were determined by ${ }^{1} \mathrm{H}$ NMR analysis using $\mathrm{CH}_{2} \mathrm{Br}_{2}$ as the internal standard.

4CzIPN $=$ 2,4,5,6-tetra( $9 H$-carbazol-9-yl)isophthalonitrile. 


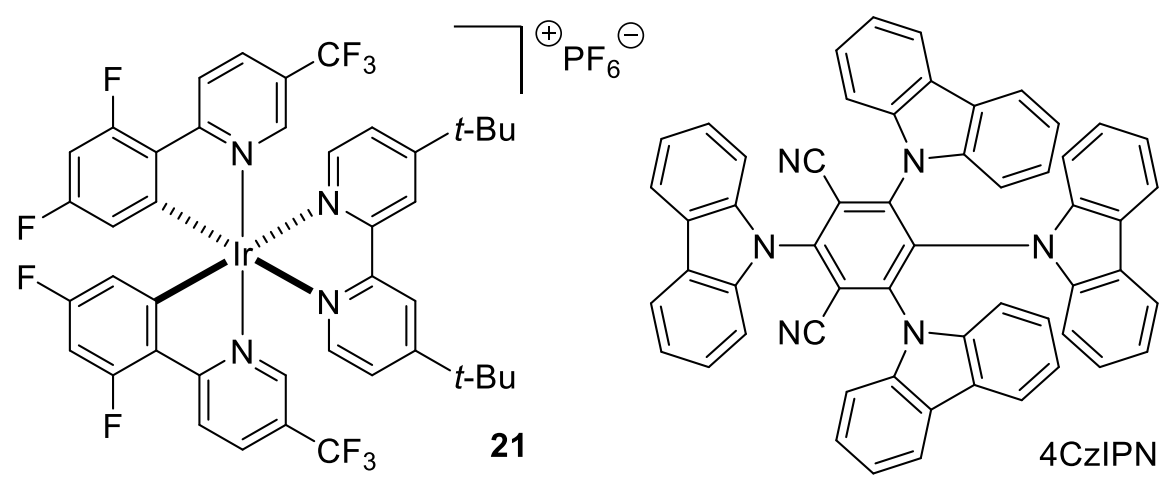

\section{Proposed mechanism of photocatalytic decarboxylative cyclization}

Scheme S1. Proposed mechanism of photocatalytic decarboxylative cyclization

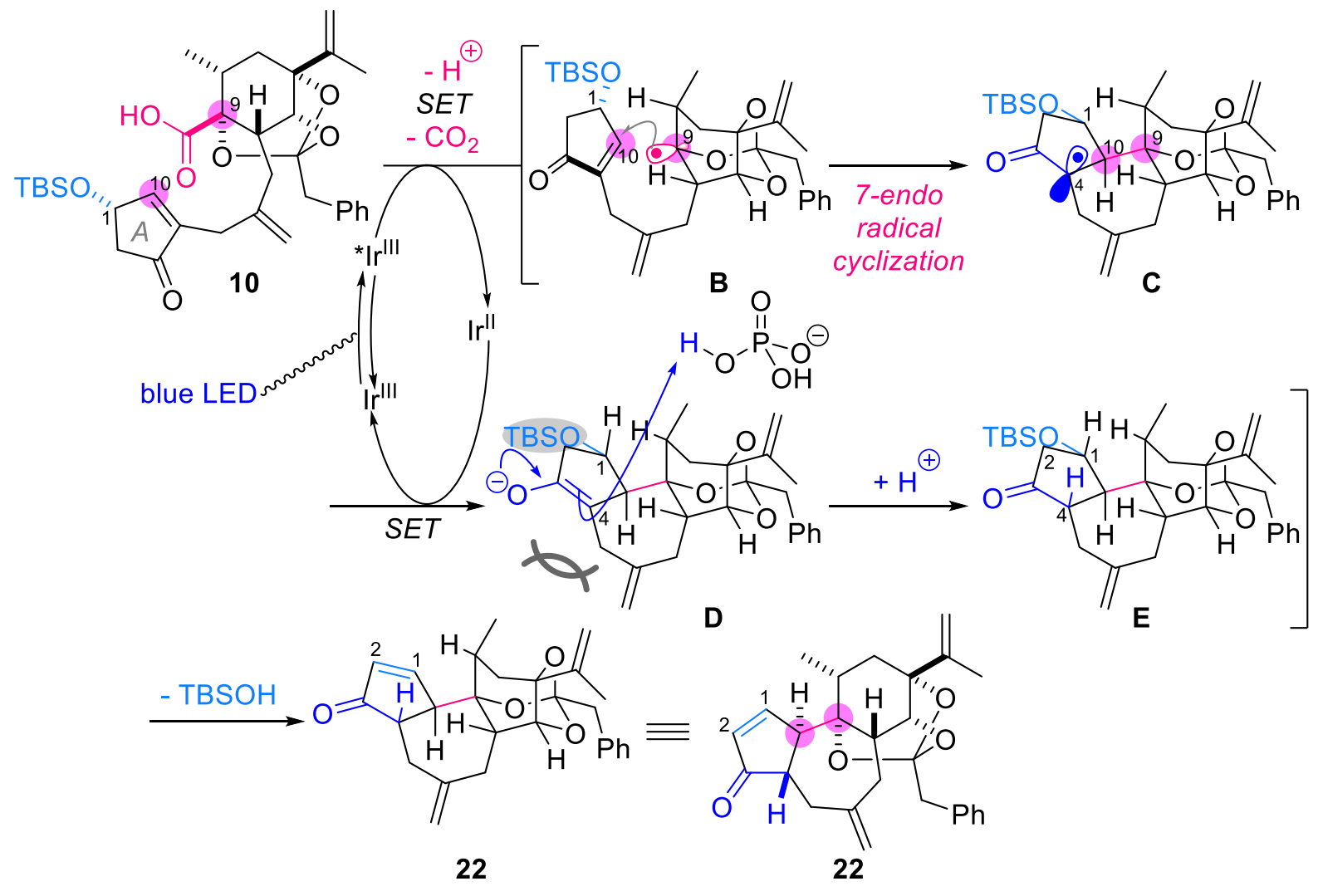




\section{Photocatalytic radical cyclization of C1-deoxy analogue S14}

Scheme S2. Photocatalytic radical cyclization of C1-deoxy analogue S14 ${ }^{a}$
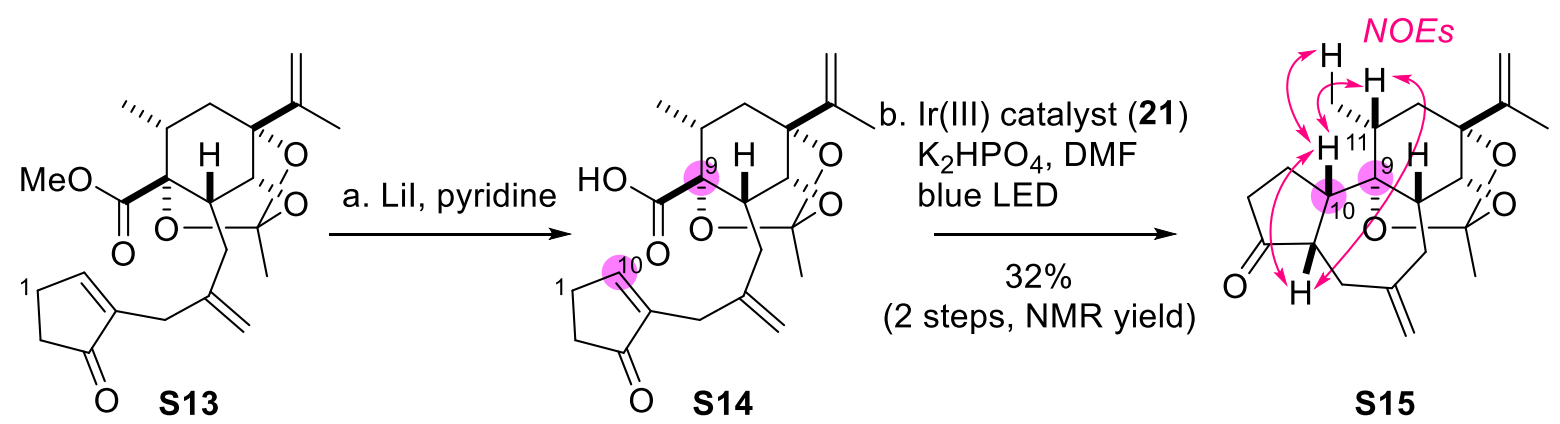

${ }^{a}$ Reaction conditions: (a) LiI ( 5 equiv), pyridine $(0.03 \mathrm{M}), 135{ }^{\circ} \mathrm{C}$. (b) 21 (20 mol\%), $\mathrm{K}_{2} \mathrm{HPO}_{4}$ (3 equiv), DMF $(0.005 \mathrm{M})$, blue LED (GrassyLeDio RX122 Deep, $23 \mathrm{~W})$. The 2-step yield of $\mathbf{S 1 5}$ was calculated to be 32\% (3.04 $\mu \mathrm{mol})$ by ${ }^{1} \mathrm{H}$ NMR analysis using $\mathrm{CH}_{2} \mathrm{Br}_{2}(1.24 \mathrm{mg}, 7.12 \mu \mathrm{mol})$ as an internal standard (Page S44).

Tricycle S15. ${ }^{1} \mathrm{H}$ NMR (500 MHz, $\left.\mathrm{C}_{6} \mathrm{D}_{6}\right): \delta 5.06(1 \mathrm{H}, \mathrm{s}, \mathrm{H} 16 \mathrm{a}), 4.89(1 \mathrm{H}, \mathrm{m}, \mathrm{H} 16 \mathrm{~b}), 4.88(1 \mathrm{H}$, s, H20a), 4.61 (1H, m, H20b), $3.81(1 \mathrm{H}, \mathrm{d}, J=2.2 \mathrm{~Hz}, \mathrm{H} 14), 3.01(1 \mathrm{H}, \mathrm{dd}, J=18.2,12.5 \mathrm{~Hz}$, H7a), 2.53 (1H, dd, $J=13.6,1.7 \mathrm{~Hz}, \mathrm{H} 5 \mathrm{a}), 2.36(1 \mathrm{H}, \mathrm{m}, \mathrm{H} 10), 2.21(1 \mathrm{H}, \mathrm{dd}, J=13.6,8.5 \mathrm{~Hz}$, H5b), 2.11-2.02 (2H, m, H2a, H7b), 1.94 (1H, m, H4), 1.88 (1H, m, H1a), 1.82-1.72 (2H, m, H1b, H2b), 1.72 (3H, s, H17), 1.71 (1H, dd, $J=14.2,7.9$ Hz, H12a), 1.69 (3H, s, CCH3), 1.63 $(1 \mathrm{H}, \mathrm{d}, J=14.2 \mathrm{~Hz}, \mathrm{H} 12 \mathrm{~b}), 1.35(1 \mathrm{H}, \mathrm{ddd}, J=12.5,2.8,2.2 \mathrm{~Hz}, \mathrm{H} 8), 1.31(1 \mathrm{H}, \mathrm{dq}, J=7.9$, $7.4 \mathrm{~Hz}, \mathrm{H} 11), 1.16(3 \mathrm{H}, \mathrm{d}, J=7.4 \mathrm{~Hz}, \mathrm{H} 18) .{ }^{13} \mathrm{C}\left\{{ }^{1} \mathrm{H}\right\}$ NMR (100 MHz, $\left.\mathrm{C}_{6} \mathrm{D}_{6}\right): \delta 219.5,147.4$, 144.6, 118.4, 112.1, 110.7, 84.7, 81.6, 79.9, 48.2, 45.4, 38.6, 38.4, 38.2, 35.4, 34.8, 32.8, 24.6, 21.5, 19.1, 18.5. HRMS (ESI-DART) m/z: $[\mathrm{M}+\mathrm{H}]^{+}$Calcd for $\mathrm{C}_{21} \mathrm{H}_{29} \mathrm{O}_{4}$ 345.2060; Found 345.2064. The C4- and C10-stereochemistries of S15 were determined by the NOESY experiment (Page S44). 


\section{Computational modeling of A}

General method: For the global analysis of the conformational isomers of radical $\mathbf{A}$, the structures were created by the conformational search using molecular mechanics simulations (MacroModel ver.10.5) ${ }^{\mathrm{S} 10}$ and the DFT calculations (Gaussian 16 program). ${ }^{\mathrm{S} 11}$ The relative Gibbs free energy $(\Delta G, 298 \mathrm{~K}, 1 \mathrm{~atm})$, computed for gas phase, was given in $\mathrm{kcal} / \mathrm{mol}$. The computational studies were conducted using FUJITSU Server PRIMERGY CX2570 M5 (Information Technology Center, Nagoya University).

\section{Optimization of A}

The conformational search of the corresponding C8-cation structure of $\mathbf{A}$ was first conducted by molecular mechanics simulation. The calculation was performed using a 10000-step Monte Carlo-based torsional sampling (MCMM) and Polak-Ribiere Conjugate Gradient (PRCG) energy minimization with an Optimized Potentials for Liquid Simulations 2005 (OPLS2005) force field (gas phase) to afford 24 conformational isomers within $10 \mathrm{kcal} / \mathrm{mol}$. All the cation structures were transferred into Gaussian 16 program and changed into the corresponding C8-radical structures. The geometry optimizations of the structures were performed at UM06-2X/6-31+G(d) level of theory (298 K, $1 \mathrm{~atm}$, gas phase) to afford 19 conformational isomers. Among them, the lowest energy structure was defined as A.

Figure S4. Energy, structure, and Cartesian coordinate of A:

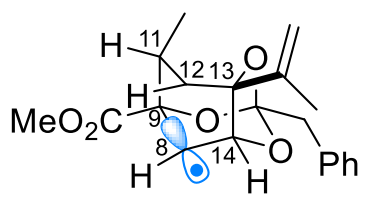

A

Gibbs free energy $(298 \mathrm{~K})=-722416.4642 \mathrm{kcal} / \mathrm{mol}$

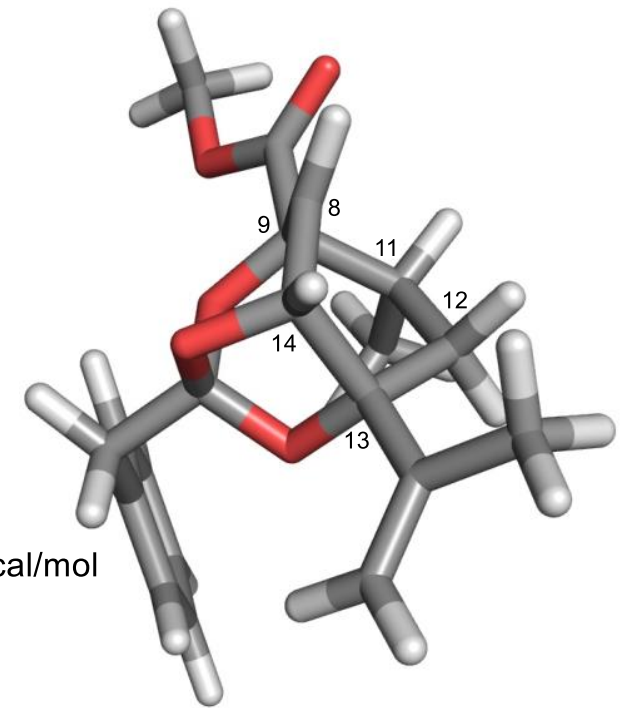

$\mathrm{C}$

$\mathrm{C}$

$\mathrm{C}$

$\mathrm{C}$

$\mathrm{C}$

$\mathrm{C}$

$\mathrm{C}$
$-0.830247$

$-0.575198$

$-1.893173$

$-2.272364$

$-2.026654$

$-1.970415$

$-3.026030$
1.143644

1.466847

1.599004

$-0.013618$

1.593046

$-0.723588$

0.191841

$-1.042664$

$-0.734550$

0.223127

0.097398

1.510230

$-1.868060$

0.274941 


\begin{tabular}{|c|c|c|c|}
\hline $\mathrm{C}$ & -4.409452 & -1.503941 & 0.750180 \\
\hline $\mathrm{C}$ & -2.694629 & -3.104872 & -0.096977 \\
\hline $\mathrm{C}$ & 0.460634 & 0.664037 & 2.132667 \\
\hline $\mathrm{O}$ & -0.711545 & -1.232637 & -0.023943 \\
\hline $\mathrm{O}$ & -1.281582 & -0.311733 & -1.954075 \\
\hline $\mathrm{O}$ & 0.340844 & 0.724838 & -0.680152 \\
\hline $\mathrm{C}$ & -0.186332 & -0.509302 & -1.106194 \\
\hline $\mathrm{C}$ & 0.924634 & -1.281972 & -1.785651 \\
\hline $\mathrm{C}$ & 2.049887 & -1.591781 & -0.827324 \\
\hline $\mathrm{C}$ & 3.024561 & -0.630951 & -0.540324 \\
\hline $\mathrm{C}$ & 4.038119 & -0.903288 & 0.375307 \\
\hline $\mathrm{C}$ & 4.089148 & -2.140399 & 1.017968 \\
\hline $\mathrm{C}$ & 3.120180 & -3.102375 & 0.739604 \\
\hline $\mathrm{C}$ & 2.107247 & -2.826492 & -0.177792 \\
\hline $\mathrm{C}$ & 0.030018 & 2.997269 & 0.008740 \\
\hline $\mathrm{O}$ & 1.326587 & 3.032126 & -0.287619 \\
\hline $\mathrm{O}$ & -0.623980 & 3.970033 & 0.307787 \\
\hline $\mathrm{C}$ & 1.921629 & 4.332024 & -0.239590 \\
\hline $\mathrm{H}$ & -1.185896 & 2.042456 & 1.984137 \\
\hline $\mathrm{H}$ & -2.533642 & 2.462524 & -0.776821 \\
\hline $\mathrm{H}$ & -3.256273 & 0.065267 & -1.493648 \\
\hline $\mathrm{H}$ & -1.821904 & -0.579372 & 2.360058 \\
\hline $\mathrm{H}$ & -2.934666 & 0.598587 & 1.653567 \\
\hline $\mathrm{H}$ & -4.412385 & -1.278219 & 1.822890 \\
\hline $\mathrm{H}$ & -4.794453 & -0.613476 & 0.236834 \\
\hline $\mathrm{H}$ & -5.108163 & -2.325459 & 0.574935 \\
\hline $\mathrm{H}$ & -3.427948 & -3.906577 & -0.079234 \\
\hline $\mathrm{H}$ & -1.686760 & -3.343069 & -0.418434 \\
\hline $\mathrm{H}$ & 1.259566 & 1.406588 & 2.026874 \\
\hline $\mathrm{H}$ & 0.285392 & 0.502053 & 3.201668 \\
\hline $\mathrm{H}$ & 0.810379 & -0.271281 & 1.688476 \\
\hline $\mathrm{H}$ & 0.478706 & -2.198698 & -2.181978 \\
\hline $\mathrm{H}$ & 1.273898 & -0.675594 & -2.626417 \\
\hline $\mathrm{H}$ & 2.968757 & 0.341666 & -1.022596 \\
\hline $\mathrm{H}$ & 4.788688 & -0.147678 & 0.589894 \\
\hline $\mathrm{H}$ & 4.880408 & -2.353071 & 1.731140 \\
\hline $\mathrm{H}$ & 3.151004 & -4.067787 & 1.236629 \\
\hline $\mathrm{H}$ & 1.345243 & -3.573995 & -0.386314 \\
\hline $\mathrm{H}$ & 1.427299 & 4.998472 & -0.949479 \\
\hline $\mathrm{H}$ & 1.834994 & 4.749414 & 0.766030 \\
\hline $\mathrm{H}$ & 2.965775 & 4.184562 & -0.509533 \\
\hline
\end{tabular}




\section{References}

S1 Hashimoto, S.; Katoh, S.; Kato, T.; Urabe, D.; Inoue, M. Total Synthesis of Resiniferatoxin Enabled by Radical Mediated Three-Component Coupling and 7-endo Cyclization. J. Am. Chem. Soc. 2017, 139, 16420-16429.

S2 McElvain, S. M.; Venerable, J. T. Ketene Acetals. XXI. The Dealcoholation of Orthoesters. Dimethylketene Dimethylacetal. J. Am. Chem. Soc. 1950, 72, 1661-1669.

S3 Song, L.; Yao, H.; Zhu, L.; Tong, R. Asymmetric Total Syntheses of (-)-Penicipyrone and (-)-Tenuipyrone via Biomimetic Cascade Intermolecular Michael Addition/Cycloketalization. Org. Lett. 2013, 15, 6-9.

S4 Danishefsky, S. J.; Cabal, M. P.; Chow, K. Novel Stereospecific Silyl Group Transfer-Reactions - Practical Routes to the Prostaglandins. J. Am. Chem. Soc. 1989, $111,3456-3457$.

S5 Vinyl ether S4 was synthesized from 2 in 17 steps. Murai, K.; Katoh, S.; Urabe, D.; Inoue, M. A Radical-Based Approach for the Construction of the Tetracyclic Structure of Resiniferatoxin. Chem. Sci. 2013, 4, 2364-2368.

S6 Orthoacetates S5 and S10 were prepared from 16 by following a similar route from 16 to orthophenylacetates $\mathbf{1 3}$ and $\mathbf{1 0}$, respectively.

S7 Because the A-value of $\mathrm{CH}=\mathrm{CH}_{2}$ (1.68) is higher than that of $\mathrm{CO}_{2} \mathrm{Me}$ (1.31), $\mathrm{C}(\mathrm{OEt})=\mathrm{CH}_{2}$ is assumed to be sterically larger than $\mathrm{CO}_{2} \mathrm{Me}$. (a) Jensen, F. R.; Bushweller, C. H.; Beck, B. H. Conformational Preferences in Monosubstituted Cyclohexanes Determined by Nuclear Magnetic Resonance Spectroscopy. J. Am. Chem. Soc. 1969, 91, 344-351. (b) Eliel, E. L.; Manoharan, M. Conformational Analysis. 40. Conformation of 1-Methyl-1-phenylcyclohexane and the Conformational Energies of the Phenyl and Vinyl Groups. J. Org. Chem. 1981, 46, 1959-1962.

S8 (a) Uoyama, H.; Goushi, K.; Shizu, K., Nomura, H. Adachi C. Highly Efficient Organic Light-Emitting Diodes from Delayed Fluorescence. Nature 2012, 492, 234-238. (b) Luo, J.; Zhang, J. Donor-Acceptor Fluorophores for Visible-Light-Promoted Organic Synthesis: Photoredox/Ni Dual Catalytic $\mathrm{C}\left(\mathrm{sp}^{3}\right)-\mathrm{C}\left(\mathrm{sp}^{2}\right)$ Cross-Coupling. ACS Catal. 2016, 6, 873-877.

S9 Application of a commonly used acridinium photocatalyst to S10 gave $\mathbf{S 1 2}$ in lower yield (18\%). Joshi-Pangu, A.; Lévesque, F.; Roth, H. G.; Oliver, S. F.; Campeau, L.-C.; Nicewicz, D.; DiRocco, D. A. Acridinium-Based Photocatalysts: A Sustainable Option in Photoredox Catalysis. J. Org. Chem. 2016, 81, 7244-7249.

S10 (a) MacroModel, version 10.5, Schrödinger, LLC, New York, NY, 2014. (b) Mohamadi, F.; Richards, N. G. J.; Guida, W. C.; Liskamp, R.; Lipton, M.; Caufield, C.; Chang, G.; 
Hendrickson, T.; Still, W. C. J. Comput. Chem. 1990, 11, 440-467.

S11 Frisch, M. J.; Trucks, G. W.; Schlegel, H. B.; Scuseria, G. E.; Robb, M. A.; Cheeseman, J. R.; Scalmani, G.; Barone, V.; Petersson, G. A.; Nakatsuji, H.; Li, X.; Caricato, M.; Marenich, A. V.; Bloino, J.; Janesko, B. G.; Gomperts, R.; Mennucci, B.; Hratchian, H. P.; Ortiz, J. V.; Izmaylov, A. F.; Sonnenberg, J. L.; Williams-Young, D.; Ding, F.; Lipparini, F.; Egidi, F.; Goings, J.; Peng, B.; Petrone, A.; Henderson, T.; Ranasinghe, D.; Zakrzewski, V. G.; Gao, J.; Rega, N.; Zheng, G.; Liang, W.; Hada, M.; Ehara, M.; Toyota, K.; Fukuda, R.; Hasegawa, J.; Ishida, M.; Nakajima, T.; Honda, Y.; Kitao, O.; Nakai, H.; Vreven, T.; Throssell, K.; Montgomery, J. A., Jr.; Peralta, J. E.; Ogliaro, F.; Bearpark, M. J.; Heyd, J. J.; Brothers, E. N.; Kudin, K. N.; Staroverov, V. N.; Keith, T. A.; Kobayashi, R.; Normand, J.; Raghavachari, K.; Rendell, A. P.; Burant, J. C.; Iyengar, S. S.; Tomasi, J.; Cossi, M.; Millam, J. M.; Klene, M.; Adamo, C.; Cammi, R.; Ochterski, J. W.; Martin, R. L.; Morokuma, K.; Farkas, O.; Foresman, J. B.; Fox, D. J. Gaussian 16 (Revision C.01), Gaussian, Inc., Wallingford CT, 2016. 


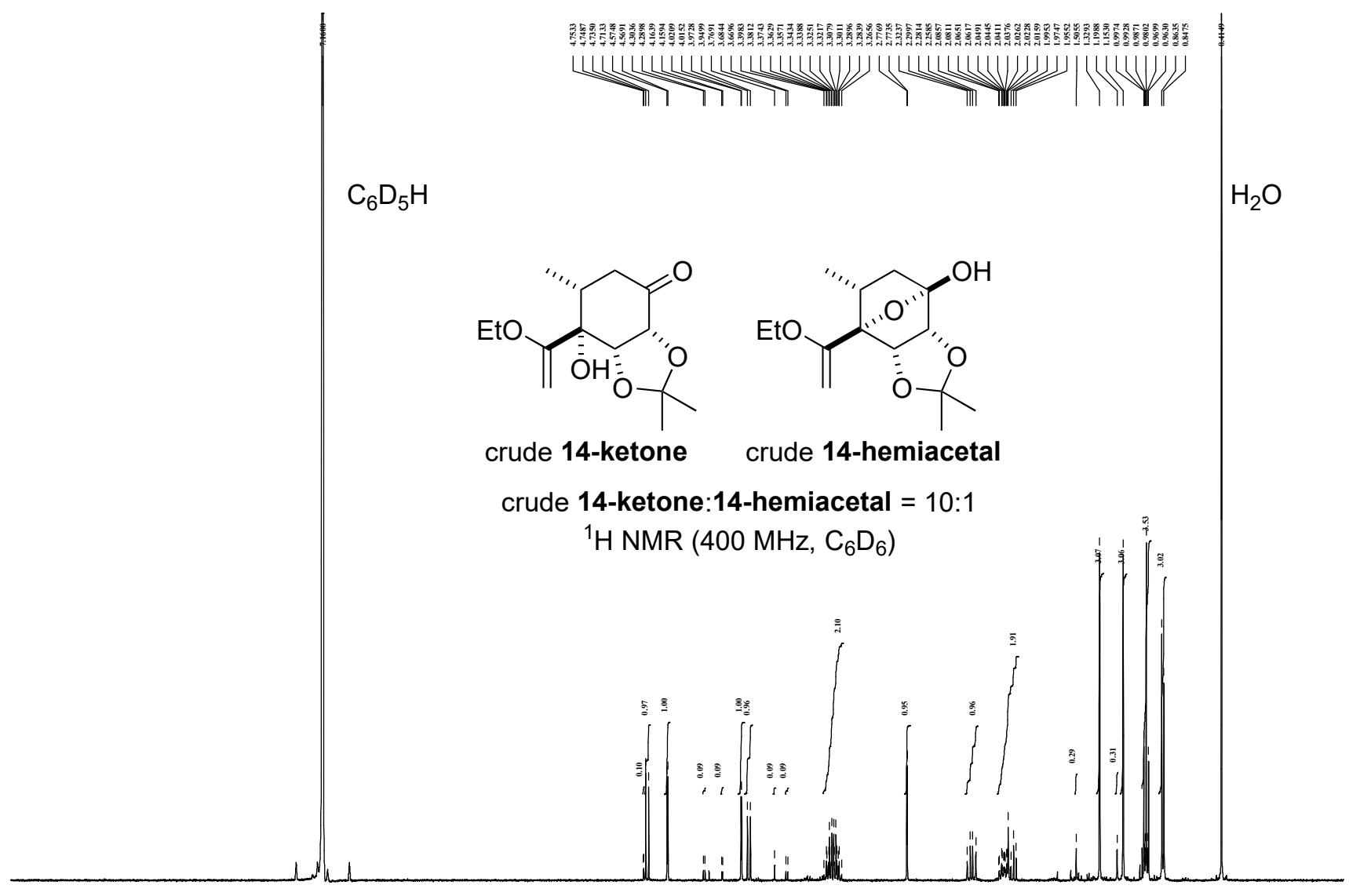

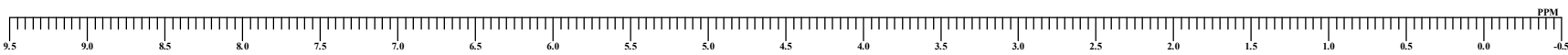




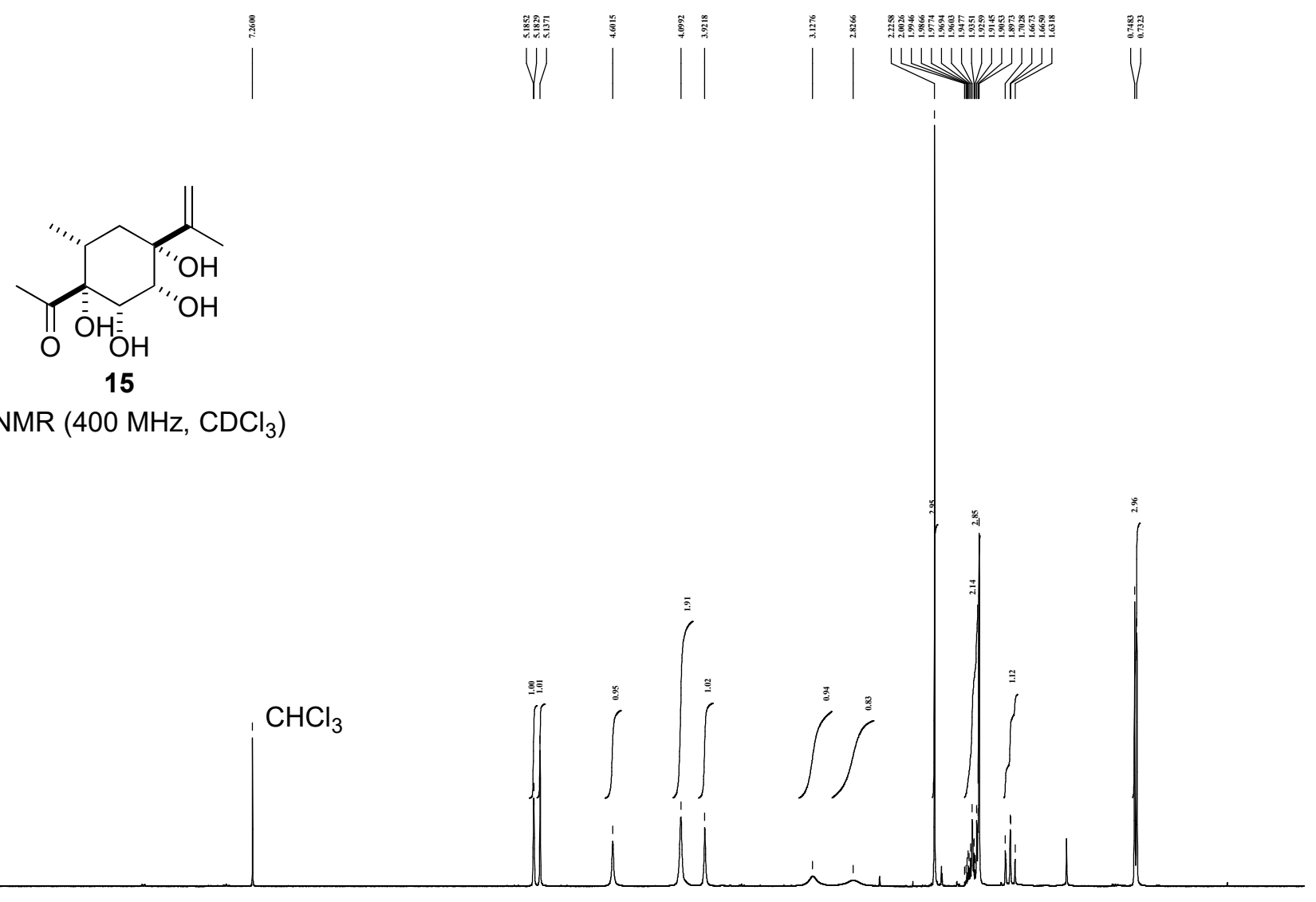

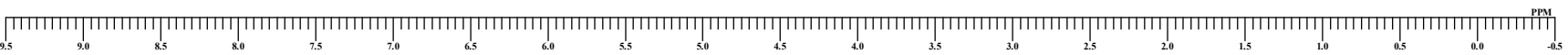

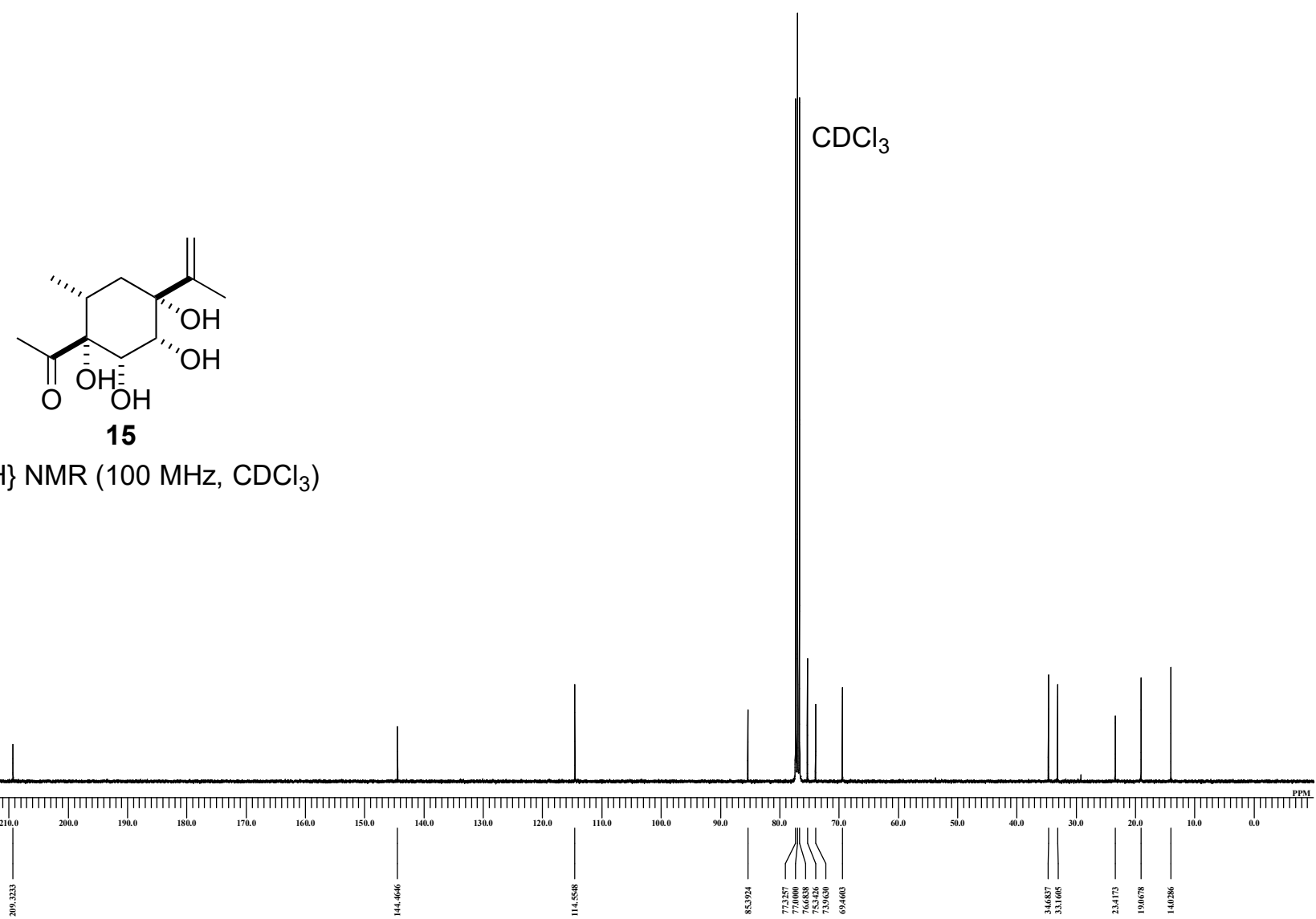




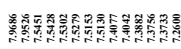

UWH

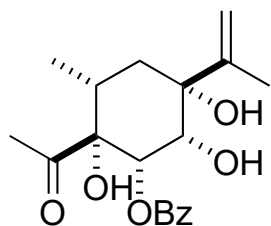

16

${ }^{1} \mathrm{H}$ NMR $\left(500 \mathrm{MHz}, \mathrm{CDCl}_{3}\right)$

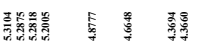

Hi

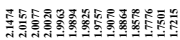

แMWUUH

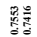

U

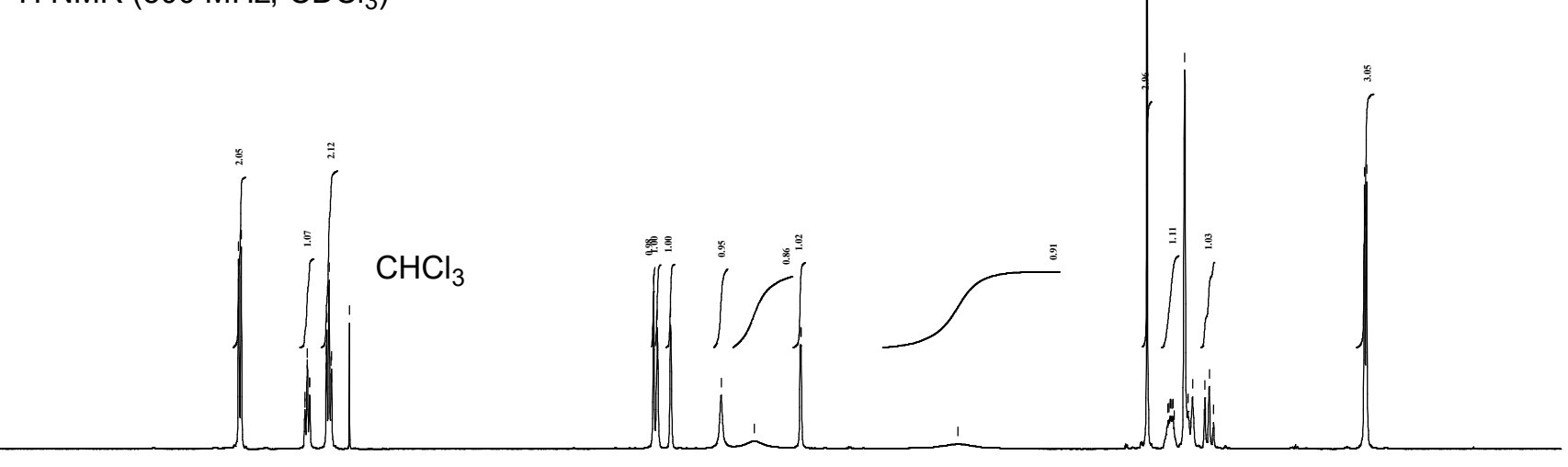

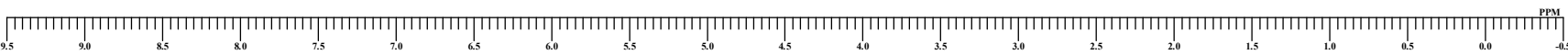




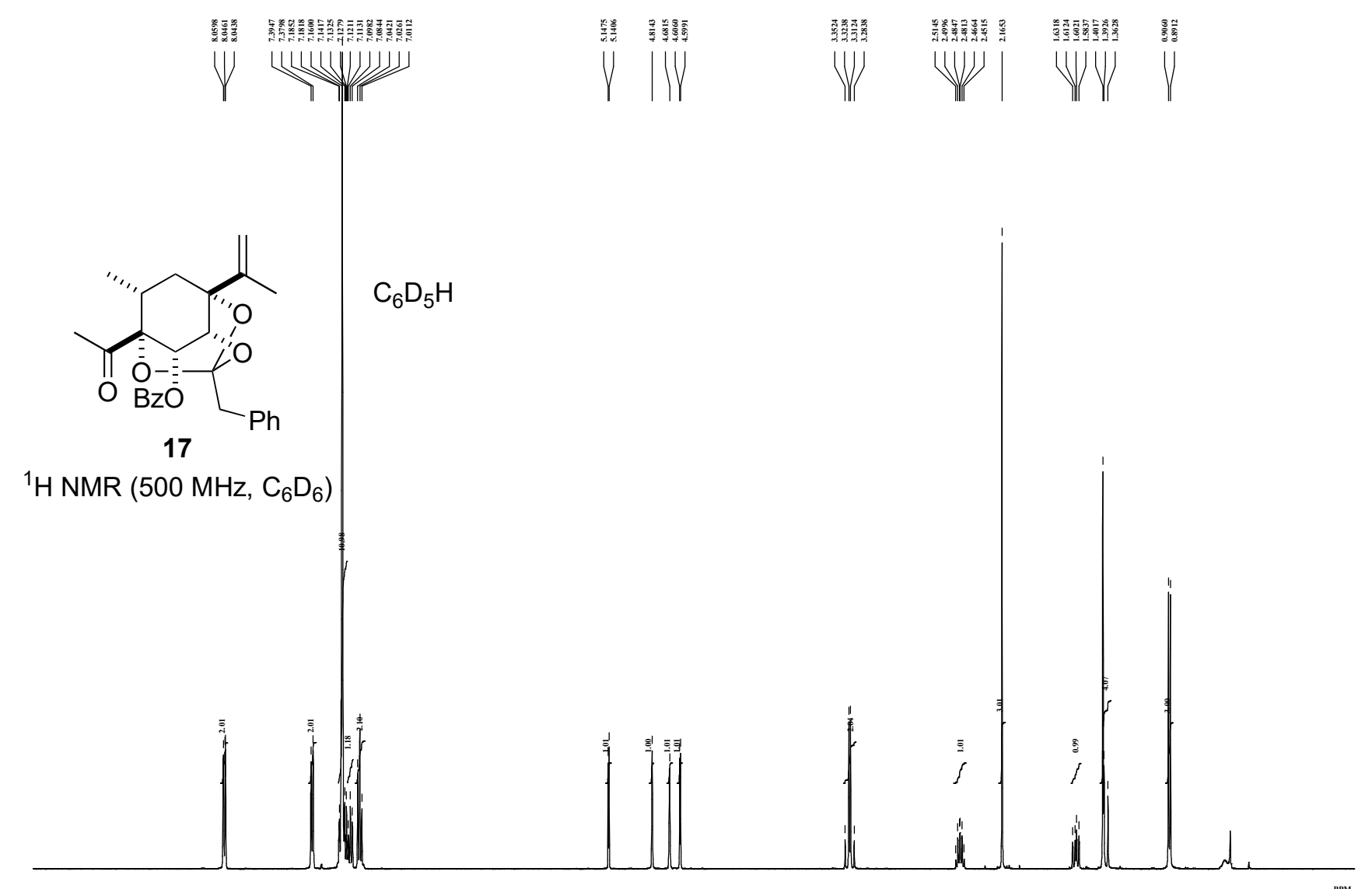

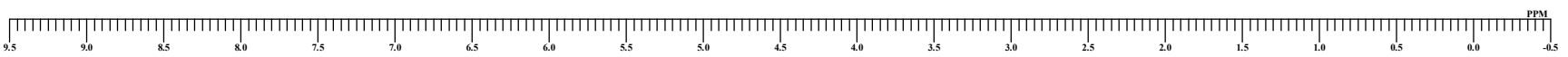

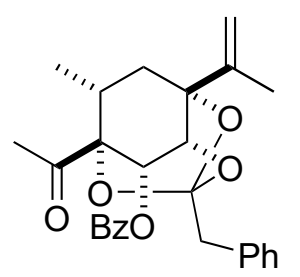

17

${ }^{13} \mathrm{C}\left\{{ }^{1} \mathrm{H}\right\}$ NMR $\left(125 \mathrm{MHz}, \mathrm{C}_{6} \mathrm{D}_{6}\right)$

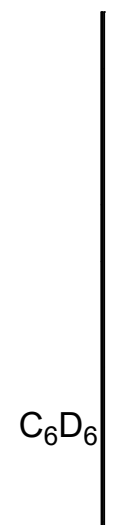




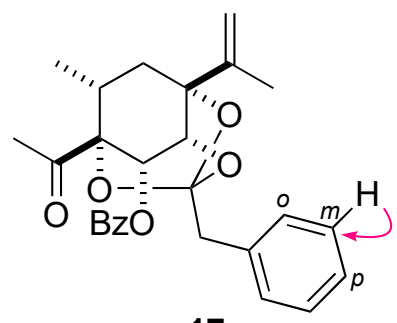

17

$\mathrm{HMQC}\left(\mathrm{C}_{6} \mathrm{D}_{6}\right)$

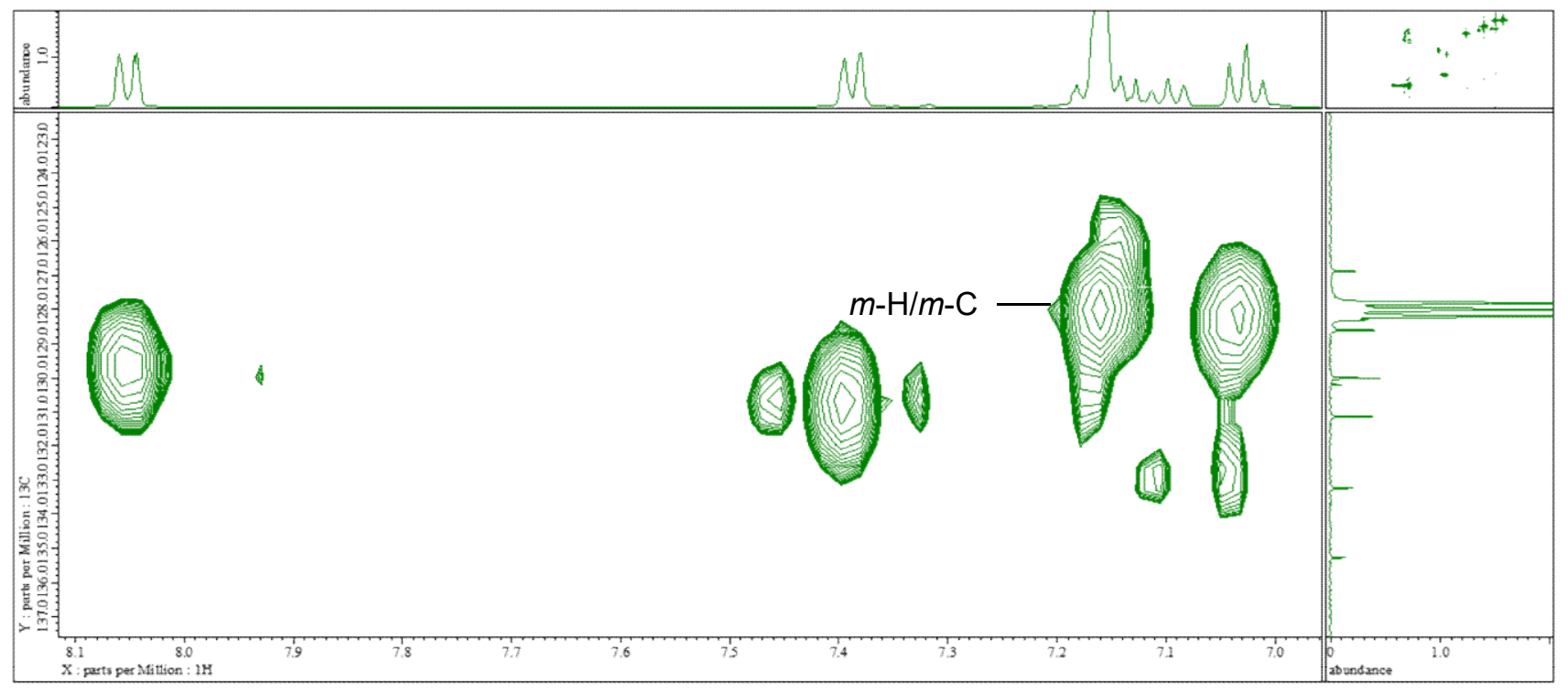



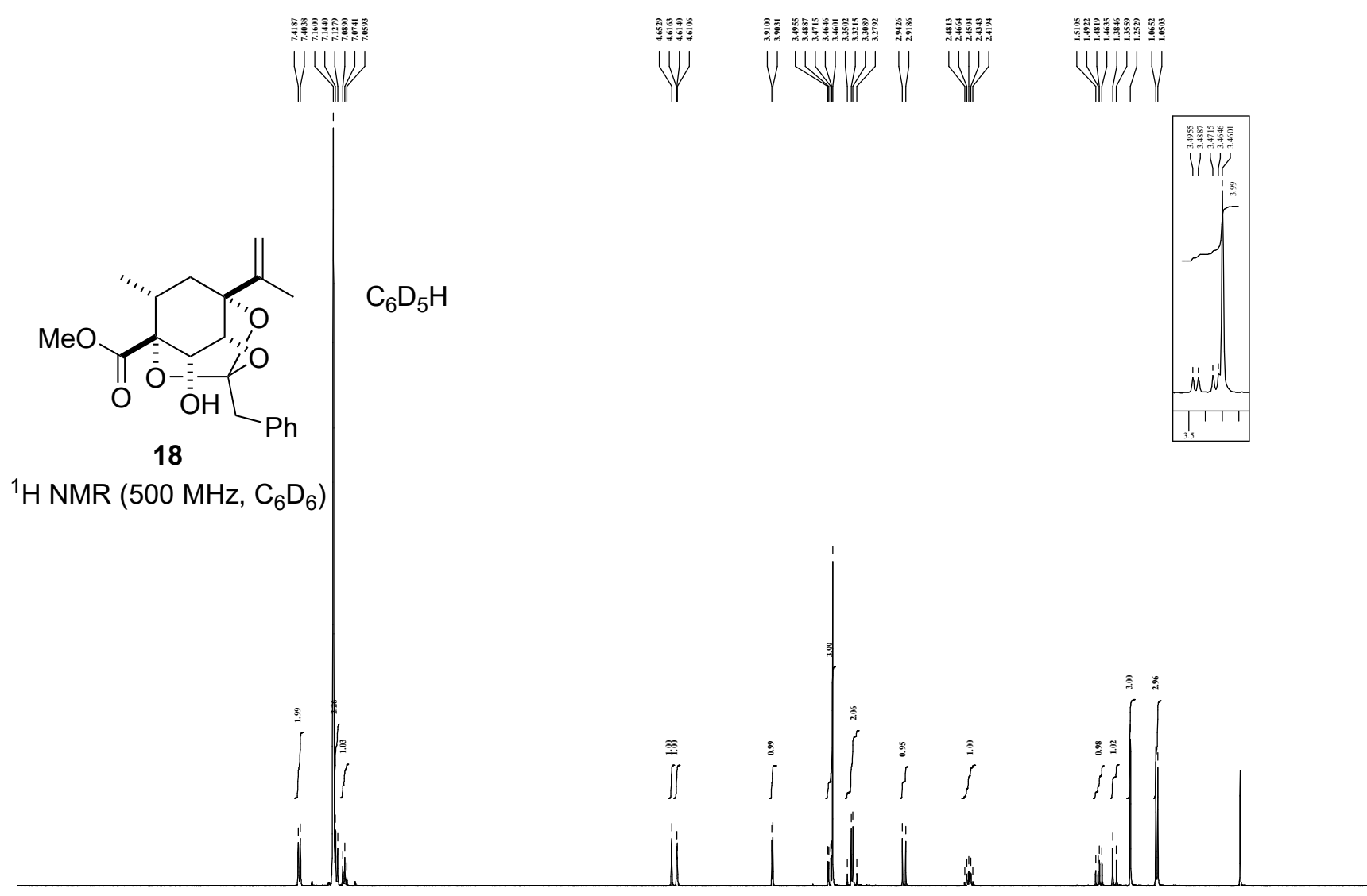

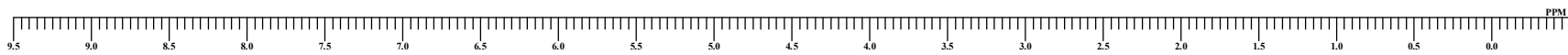
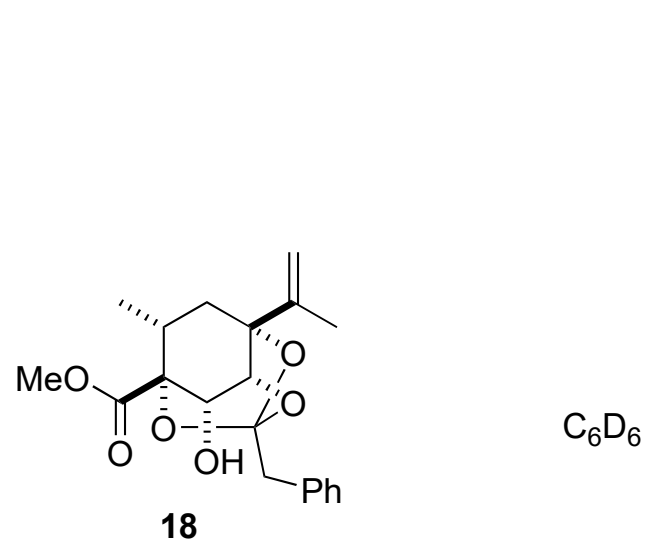

${ }^{13} \mathrm{C}\left\{{ }^{1} \mathrm{H}\right\}$ NMR $\left(125 \mathrm{MHz}, \mathrm{C}_{6} \mathrm{D}_{6}\right)$

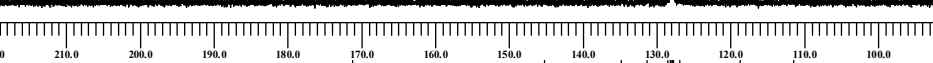

${ }^{1} \mathrm{H}$ NMR $\left(500 \mathrm{MHz}, \mathrm{C}_{6}\right.$ 


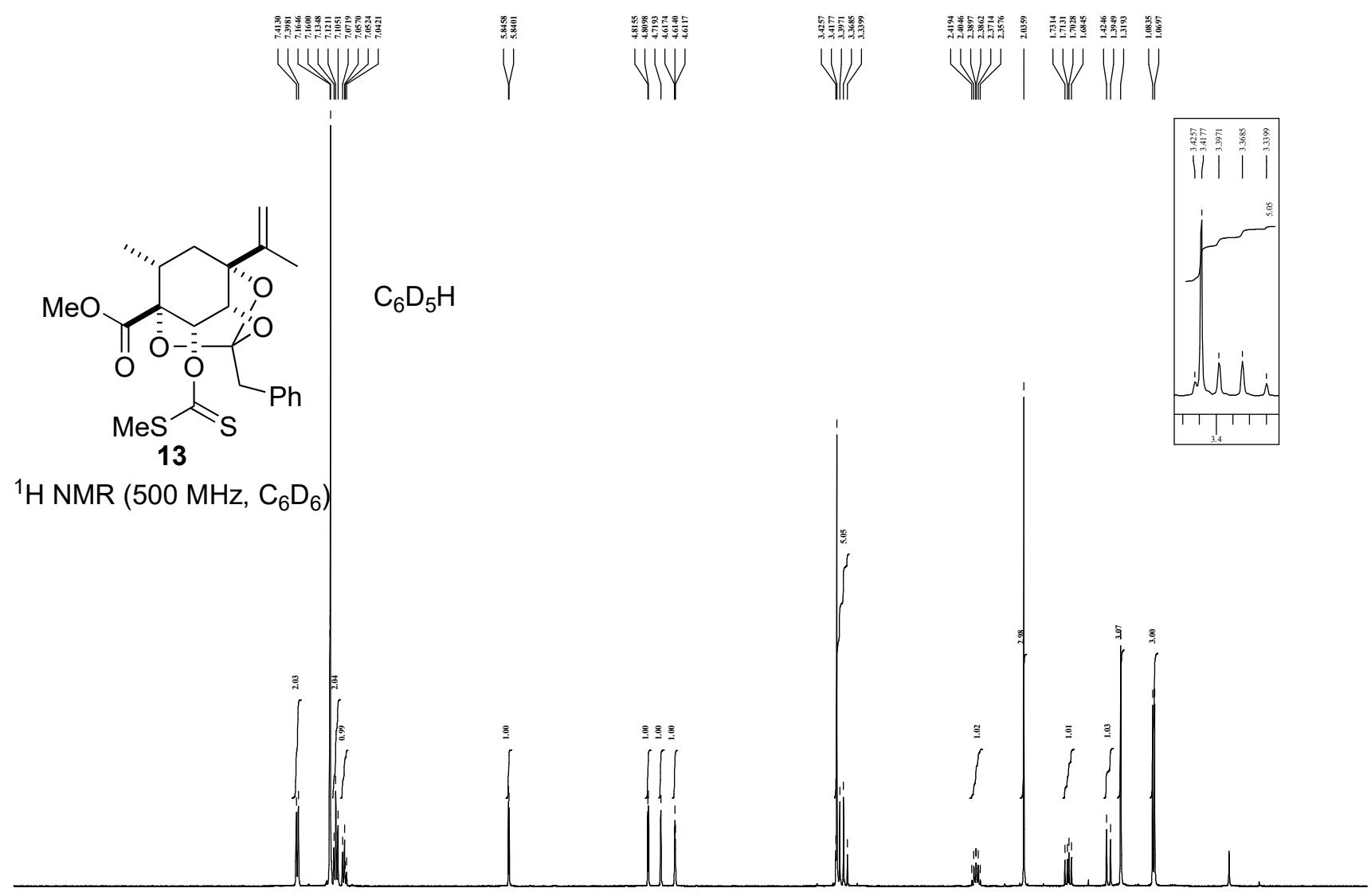

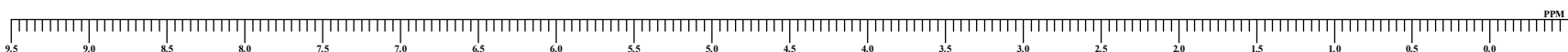
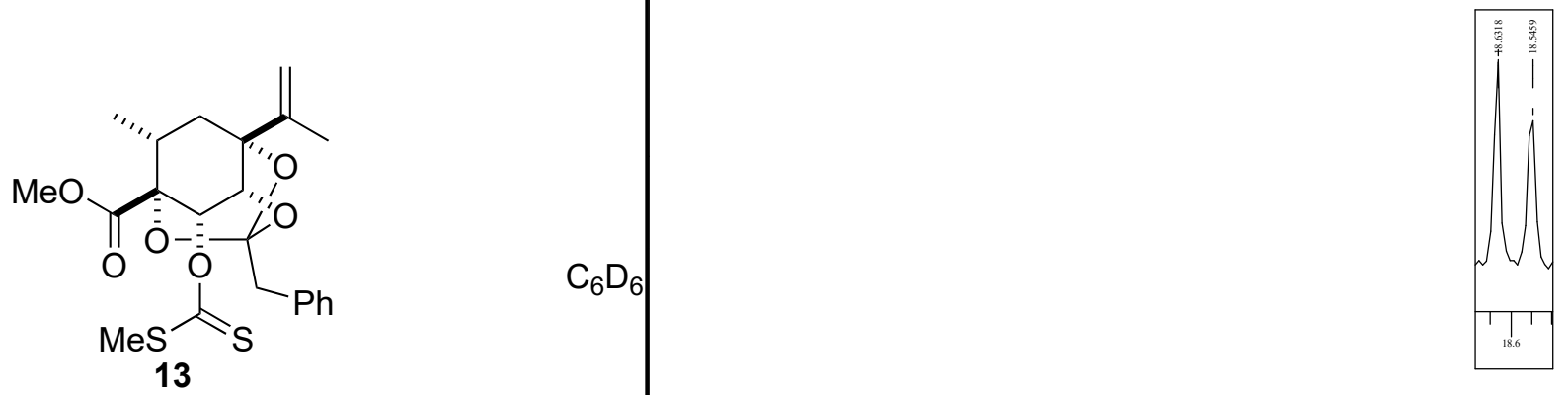

${ }^{13} \mathrm{C}\left\{{ }^{1} \mathrm{H}\right\}$ NMR $\left(125 \mathrm{MHz}, \mathrm{C}_{6} \mathrm{D}_{6}\right)$

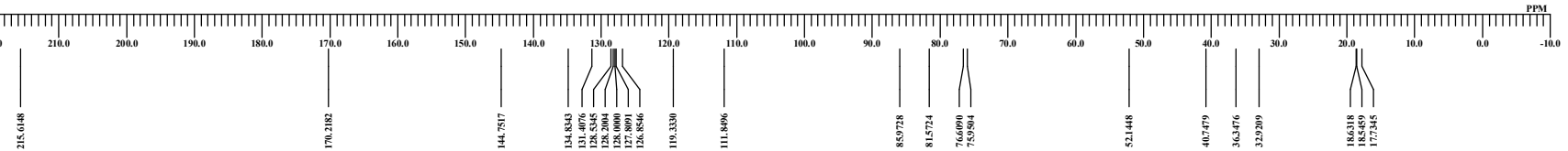




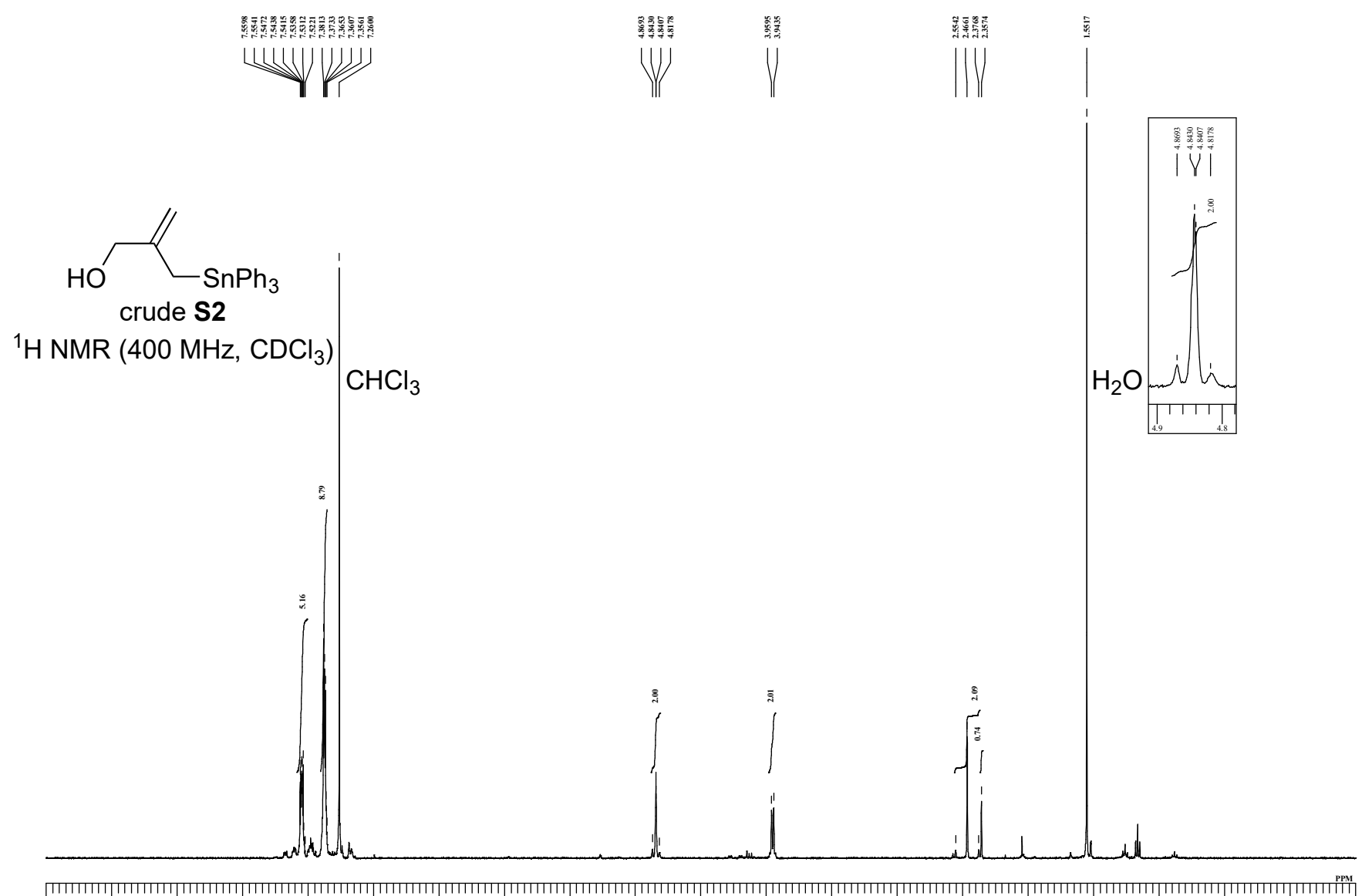


<smiles>C=C(C[SbH2])COC(C)=O</smiles>

11

${ }^{1} \mathrm{H}$ NMR (500 MHz, $\left.\mathrm{CDCl}_{3}\right)$
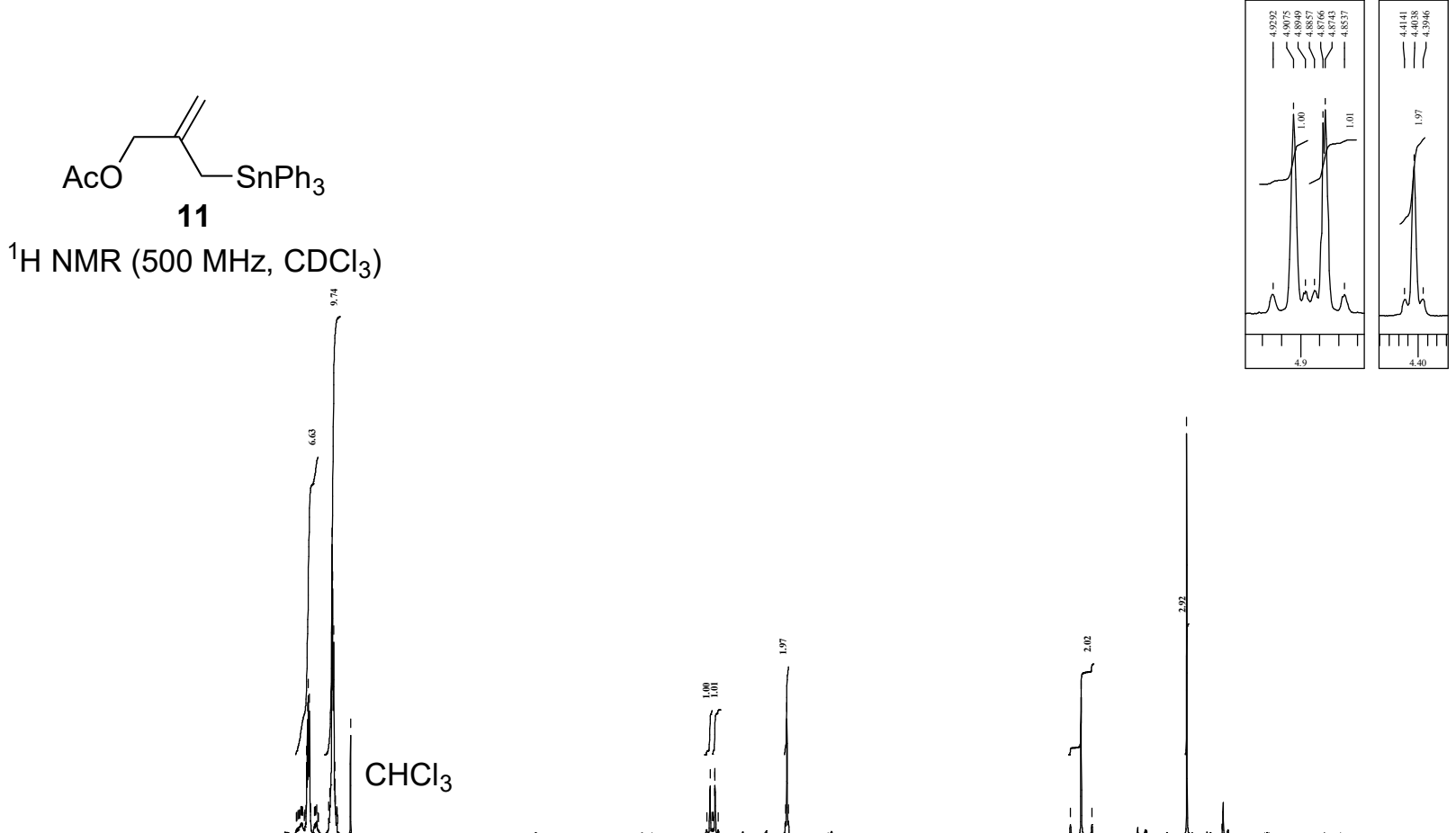

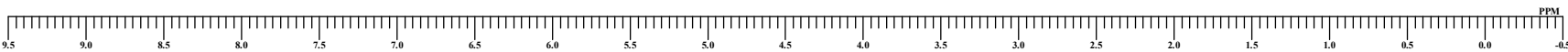

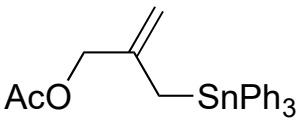

$\mathrm{CDCl}_{3}$

11

$\left.{ }^{13} \mathrm{C}\left\{{ }^{1} \mathrm{H}\right\} \mathrm{NMR}(125 \mathrm{MHz}, \mathrm{CDCl})_{3}\right)$
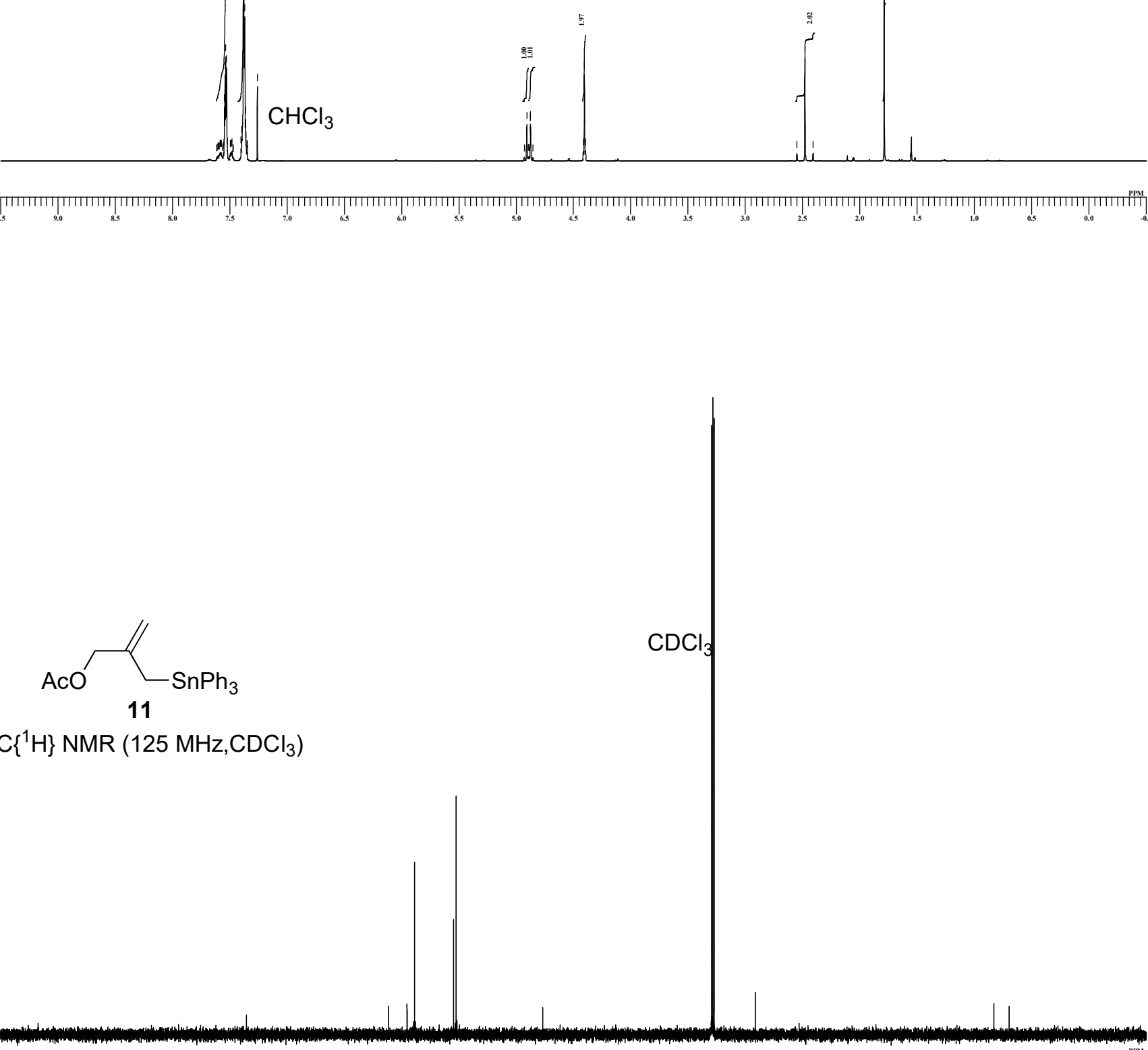

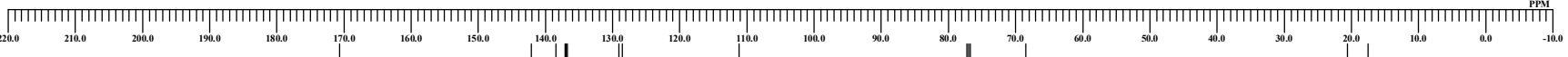

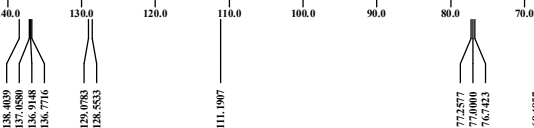




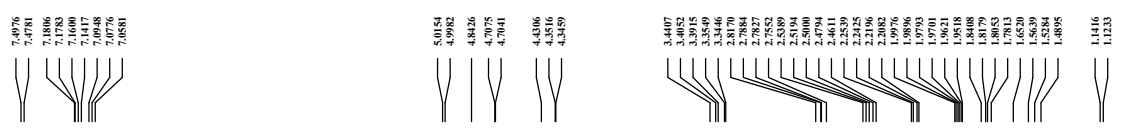

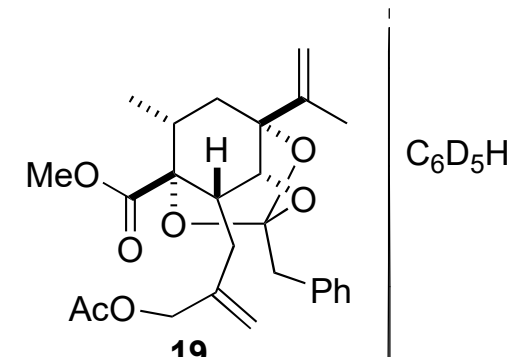

\section{${ }^{1} \mathrm{H}$ NMR $\left(500 \mathrm{MHz}, \mathrm{C}_{6} \mathrm{D}_{6}\right)$}
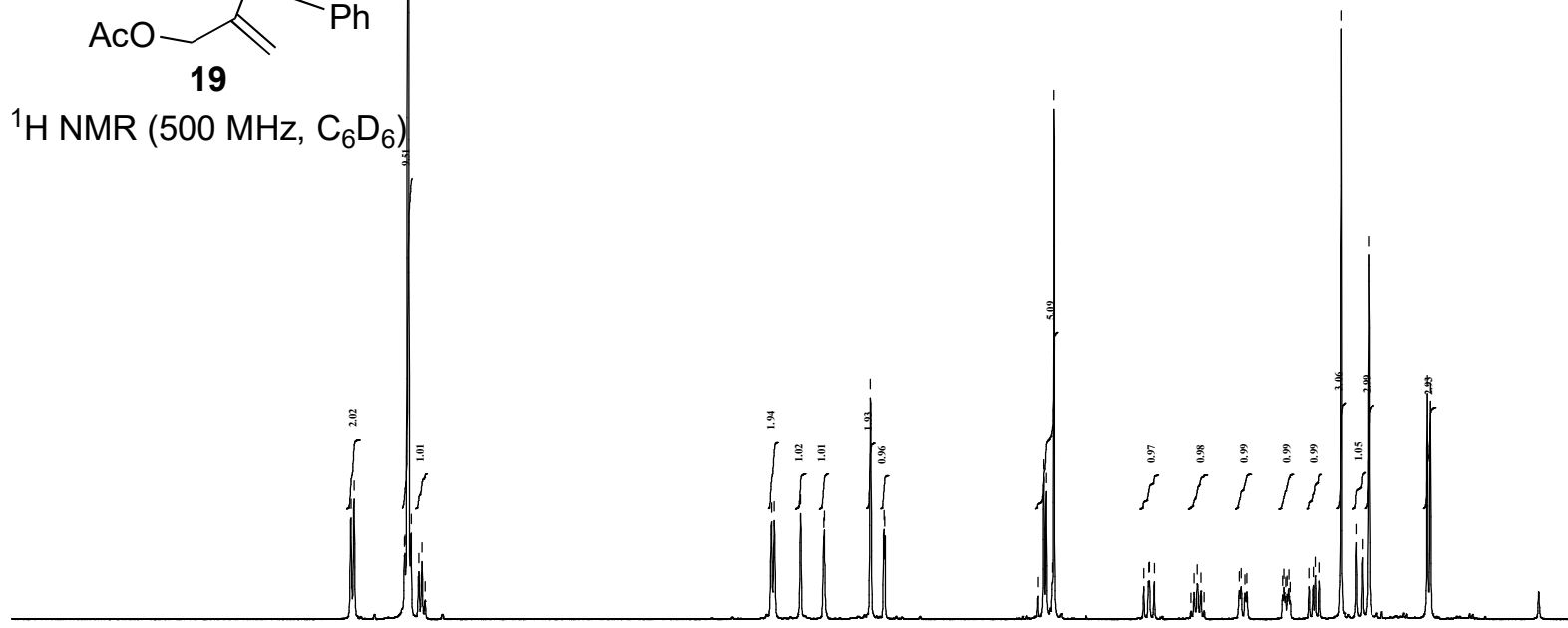

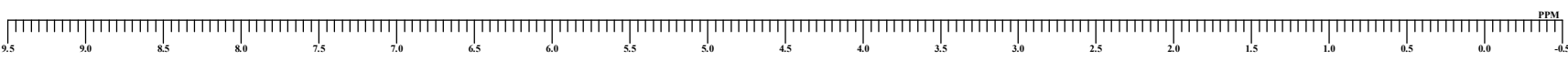

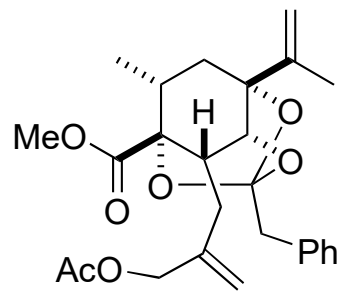<smiles></smiles>

19

${ }^{13} \mathrm{C}\left\{{ }^{1} \mathrm{H}\right\}$ NMR $\left(125 \mathrm{MHz}, \mathrm{C}_{6} \mathrm{D}_{6}\right)$

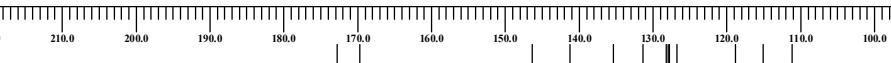

220.0
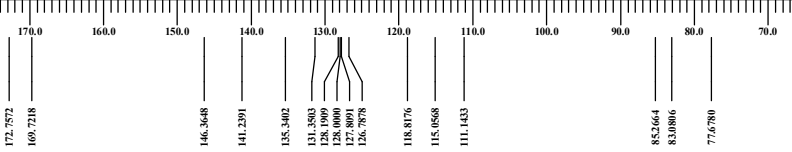

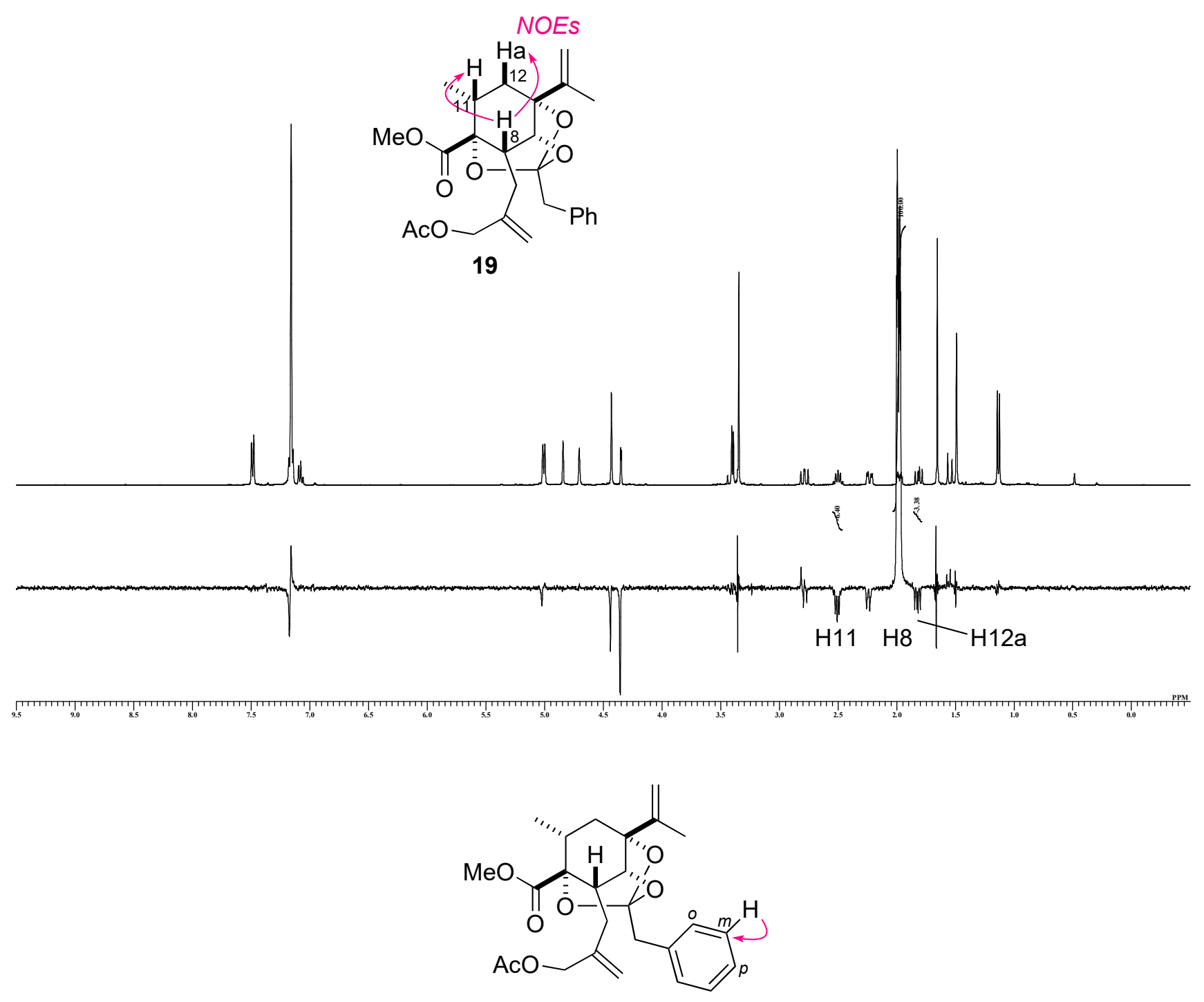

19

$\operatorname{HMQC}\left(\mathrm{C}_{6} \mathrm{D}_{6}\right)$

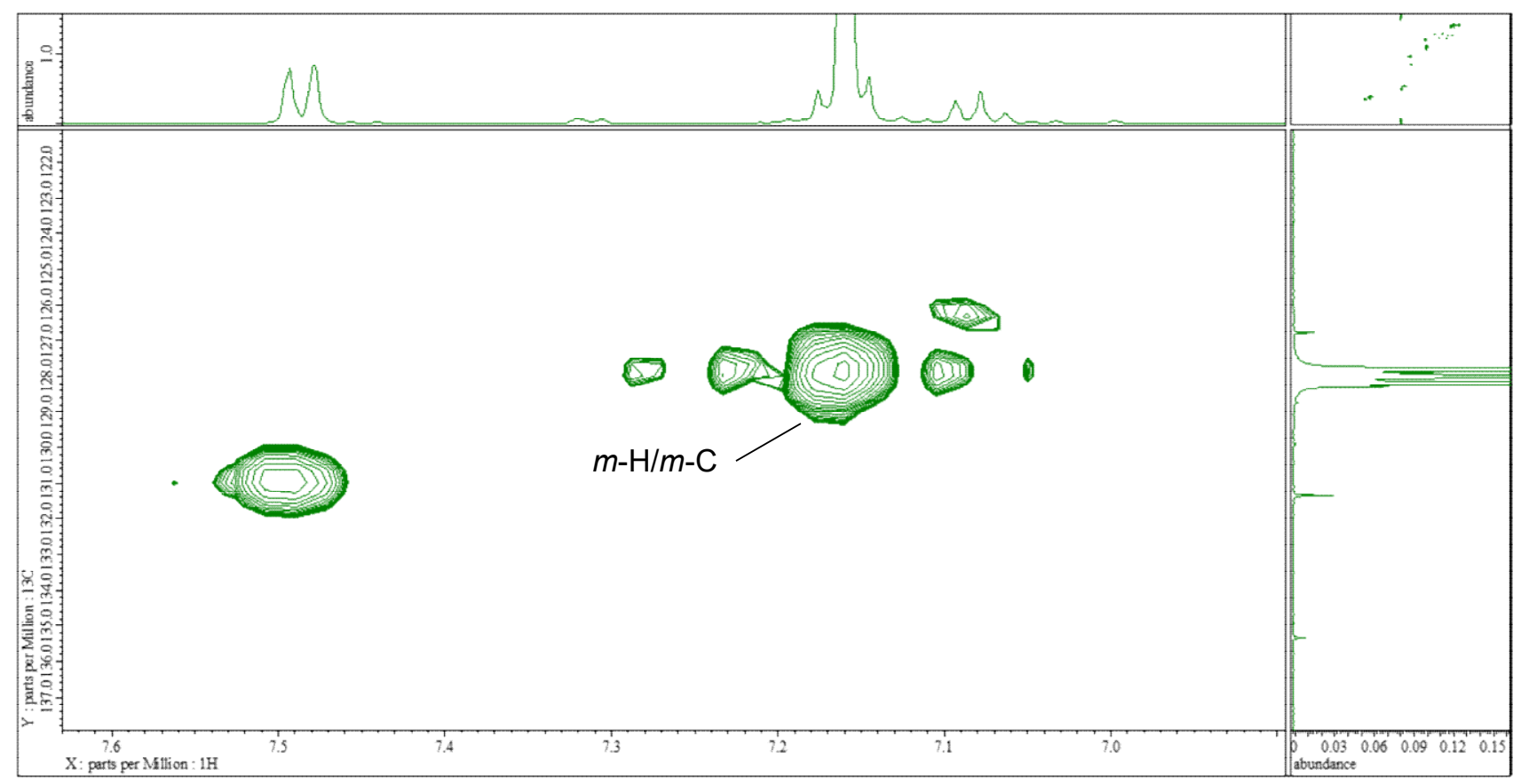


TBSO<smiles>O=C1CCC=C1I</smiles>

S3

${ }^{1} \mathrm{H}$ NMR $\left(400 \mathrm{MHz}, \mathrm{CDCl}_{3}\right)$

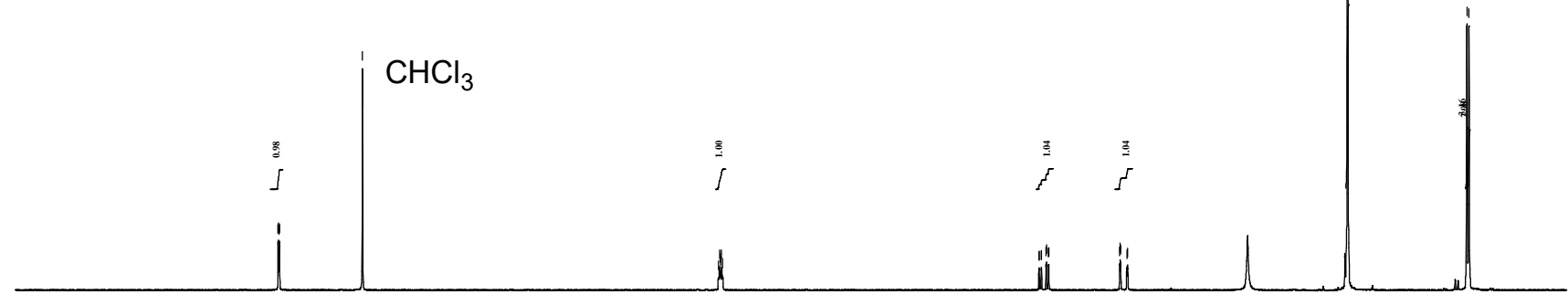

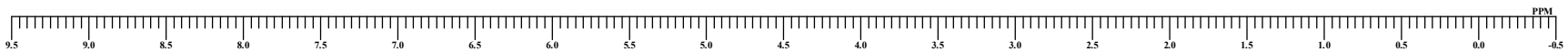




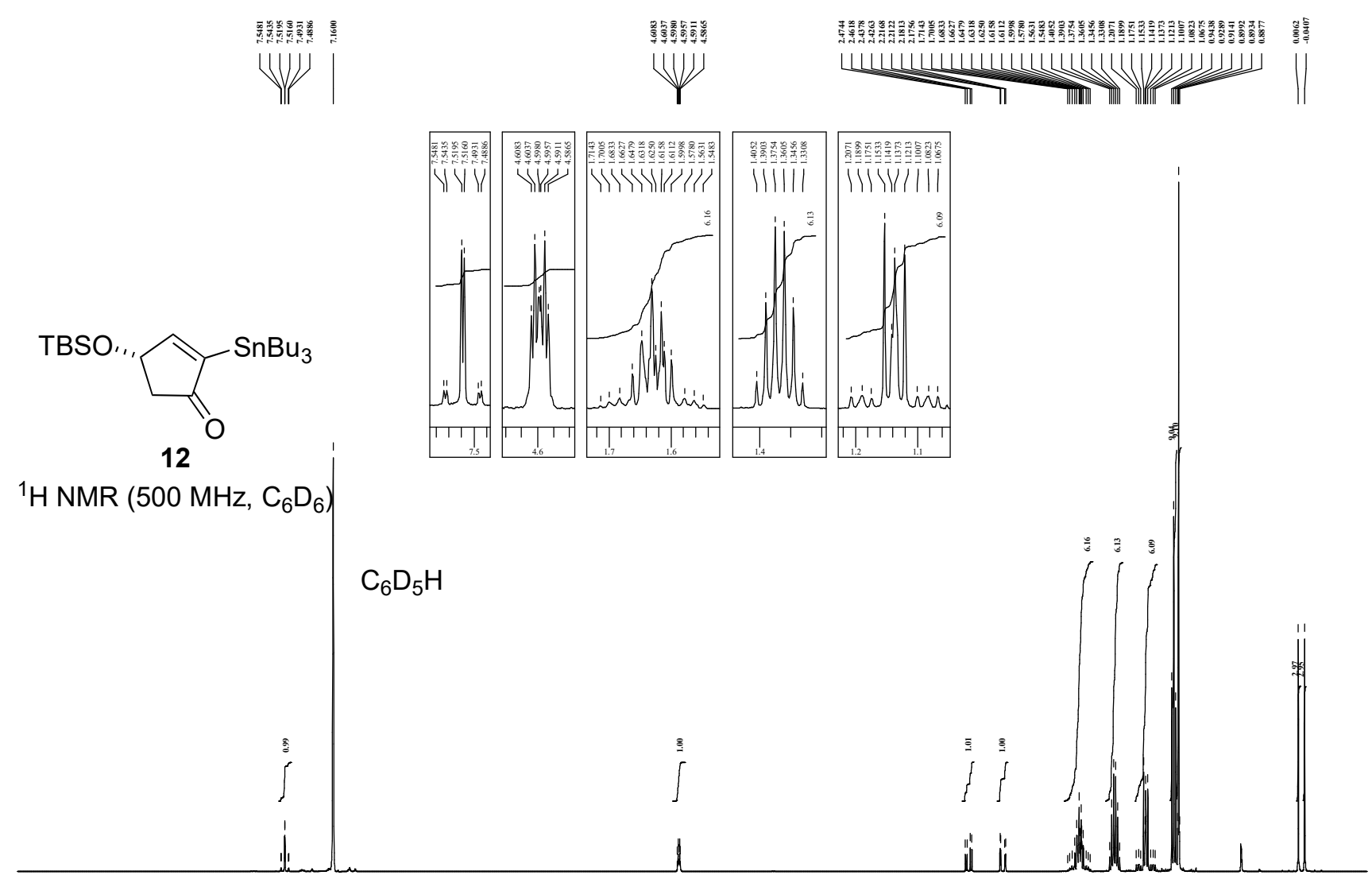

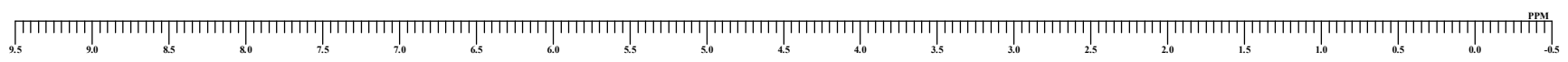

TBSO". $\mathrm{SnBu}_{3}^{\mathrm{SnB}}$

12

${ }^{13} \mathrm{C}\left\{{ }^{1} \mathrm{H}\right\} \operatorname{NMR}\left(125 \mathrm{MHz}, \mathrm{C}_{6} \mathrm{D}_{6}\right)$
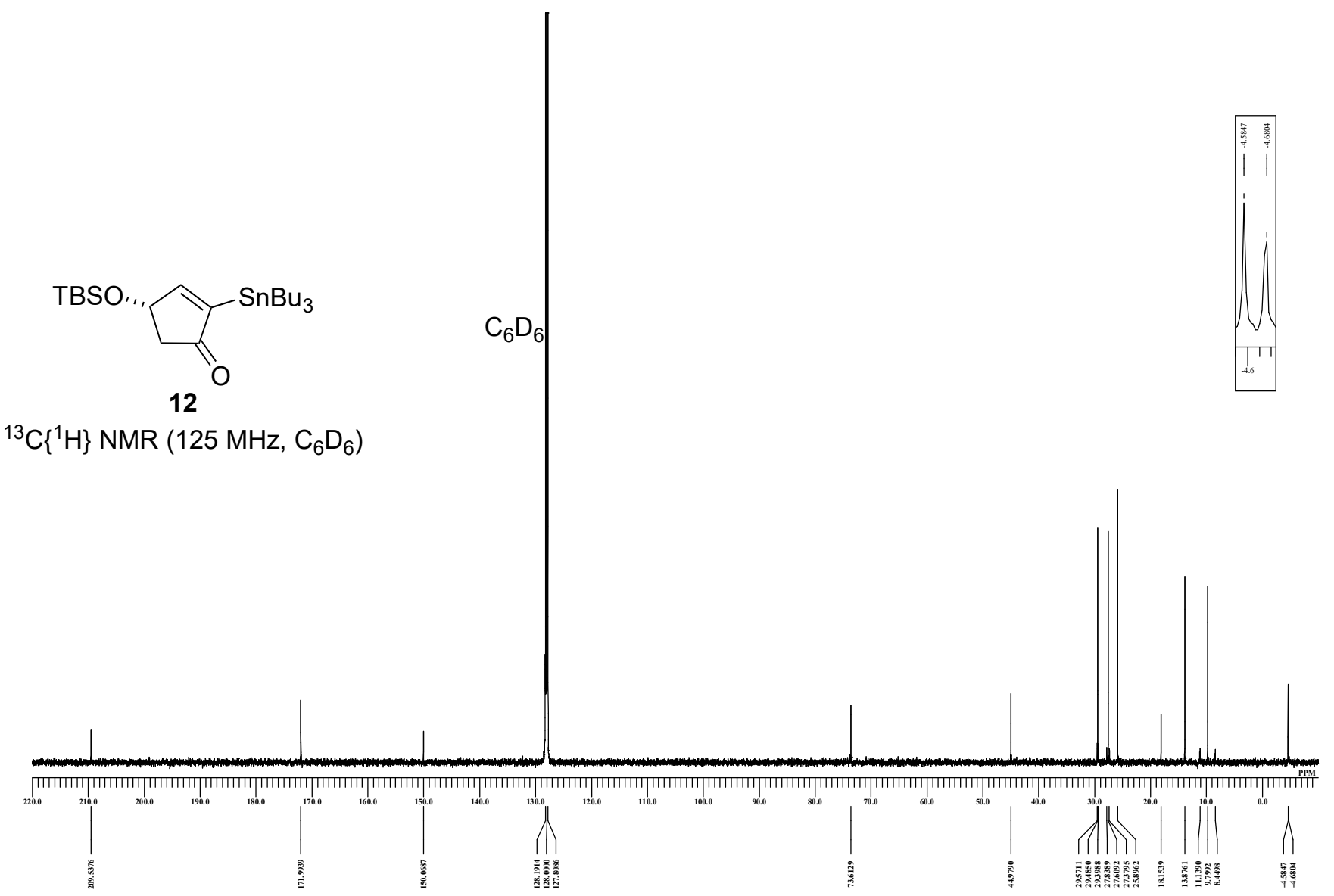


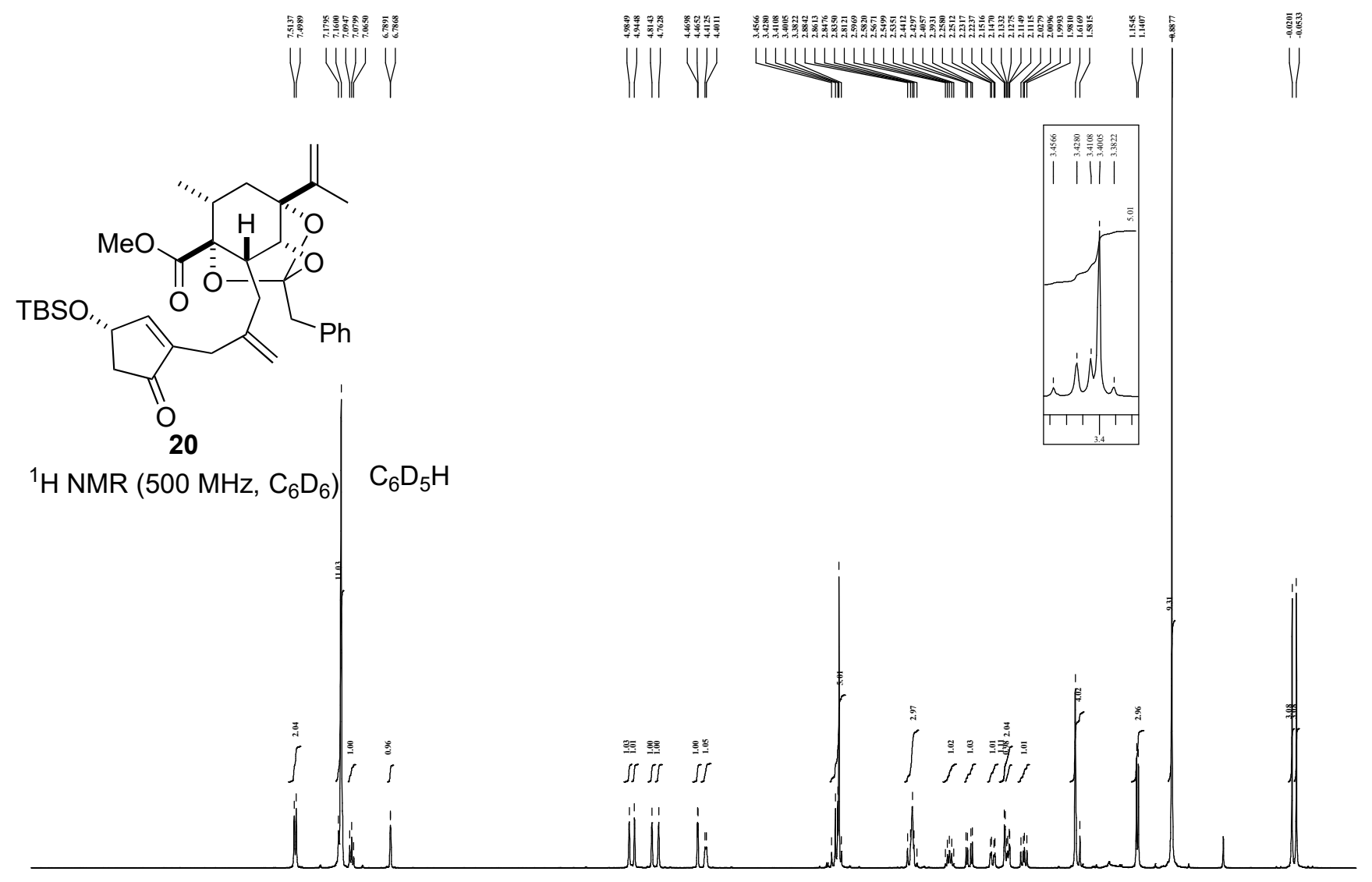

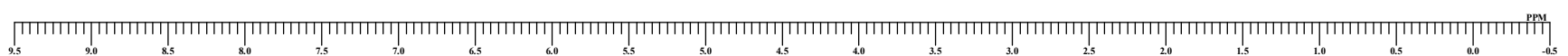
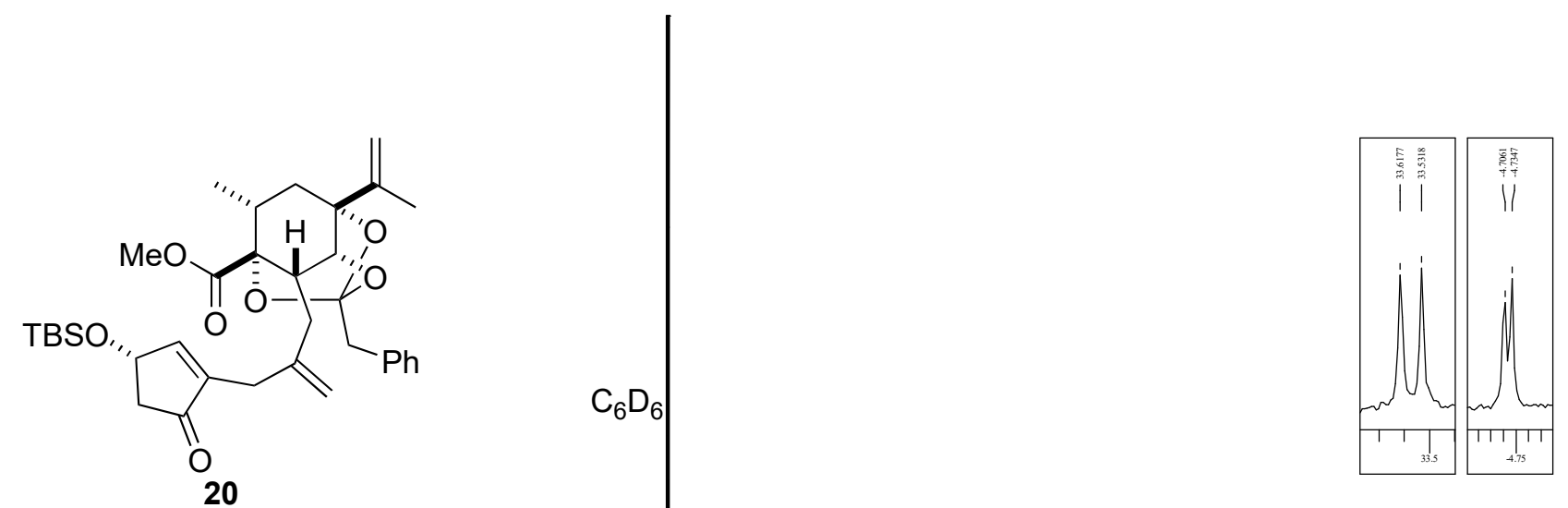

${ }^{13} \mathrm{C}\left\{{ }^{1} \mathrm{H}\right\}$ NMR $\left(125 \mathrm{MHz}, \mathrm{C}_{6} \mathrm{D}_{6}\right)$

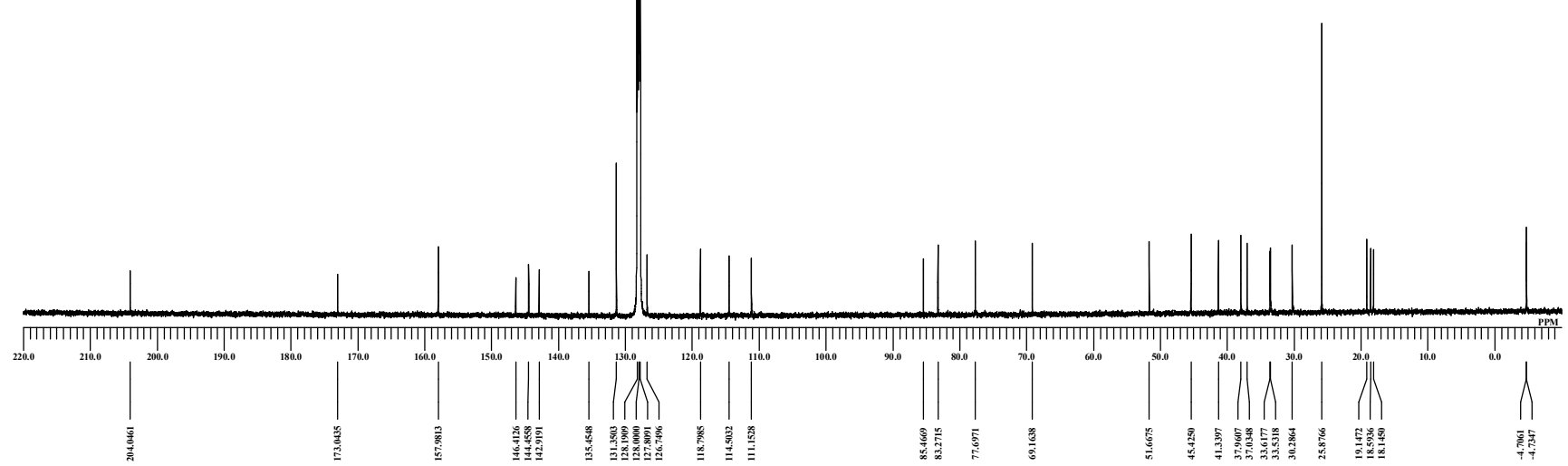



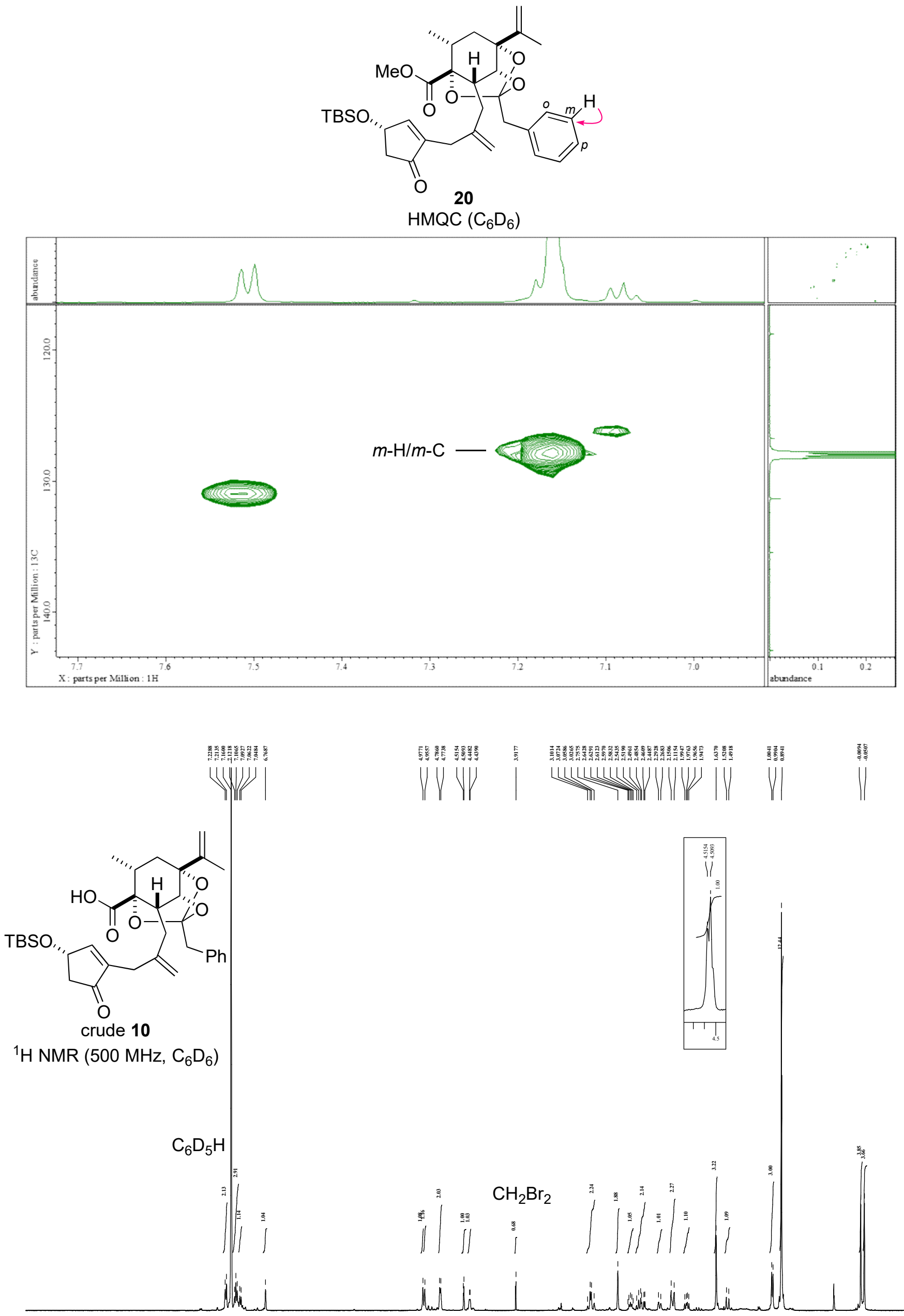

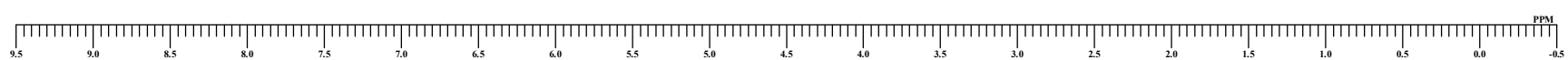




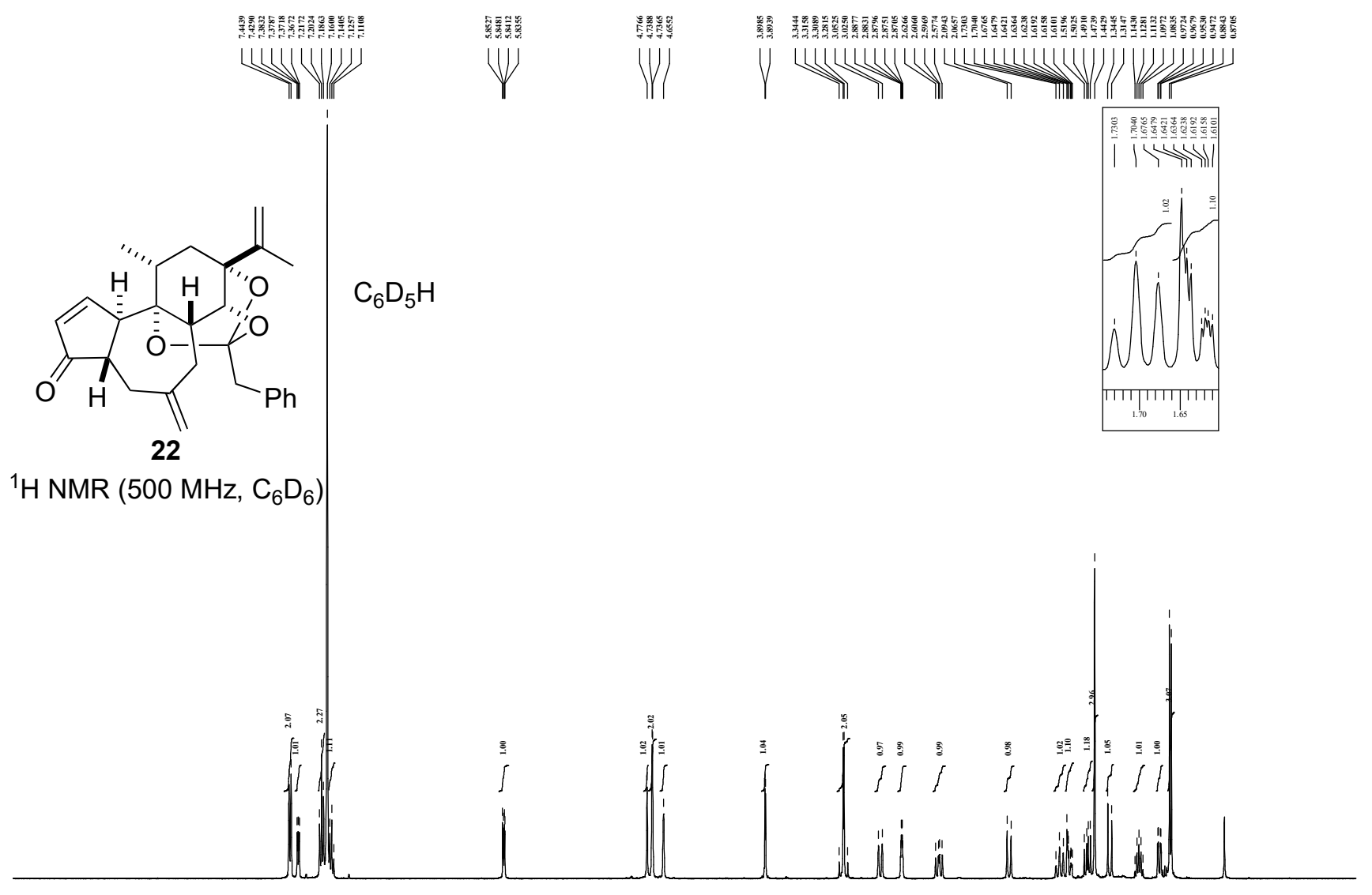

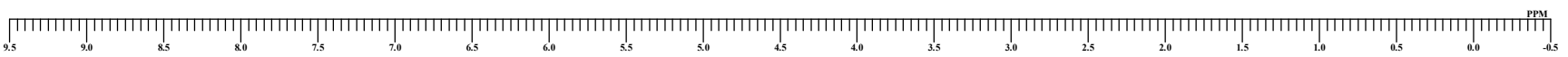
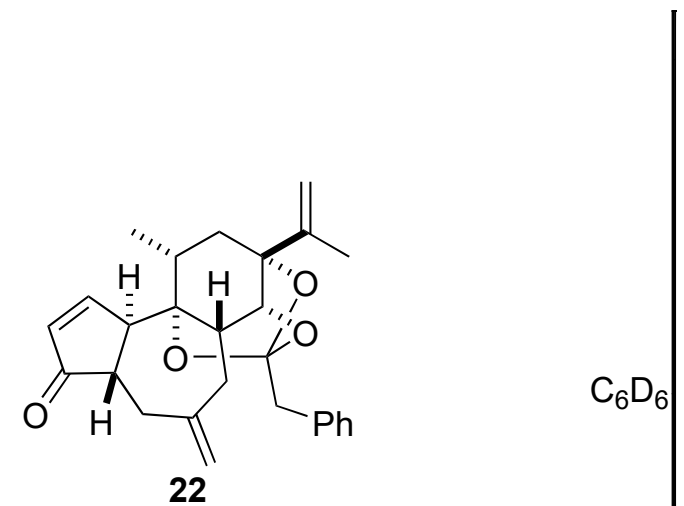

${ }^{13} \mathrm{C}\left\{{ }^{1} \mathrm{H}\right\}$ NMR $\left(125 \mathrm{MHz}, \mathrm{C}_{6} \mathrm{D}_{6}\right)$ 

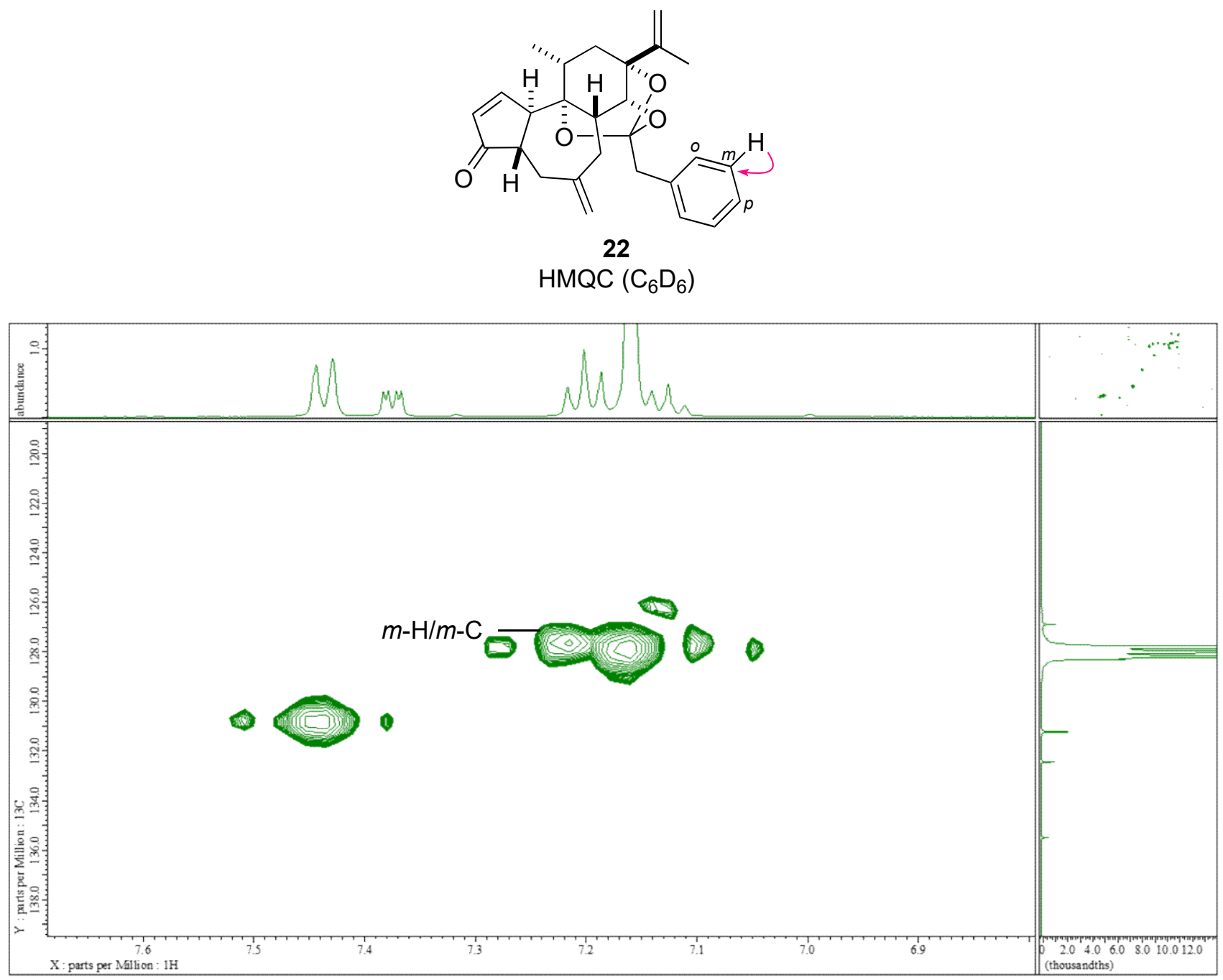

S40 


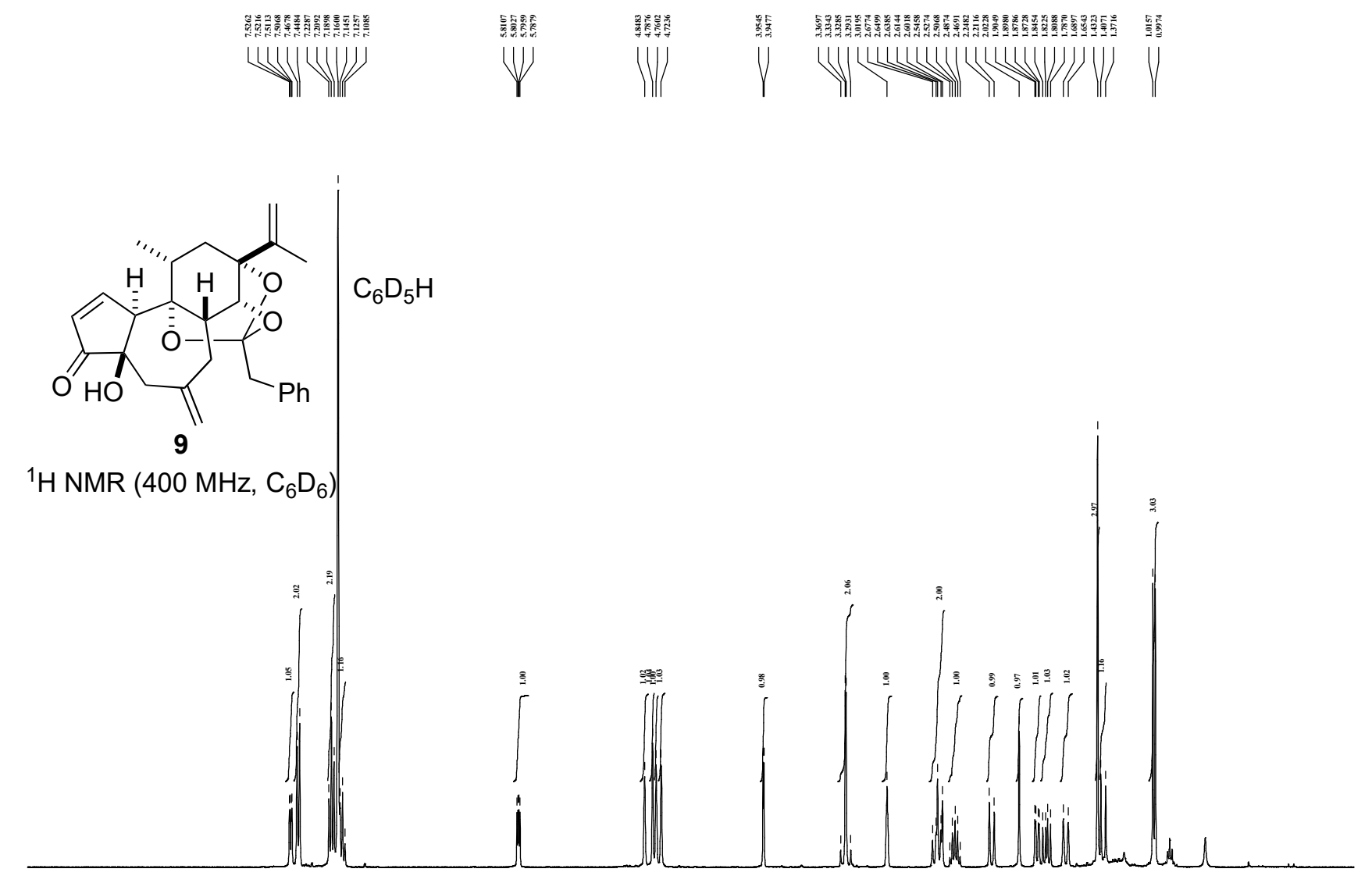

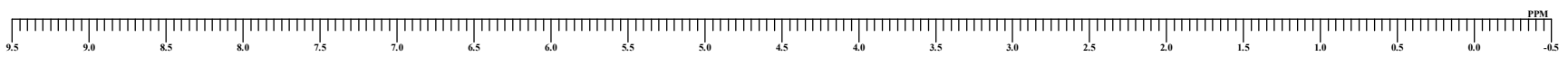
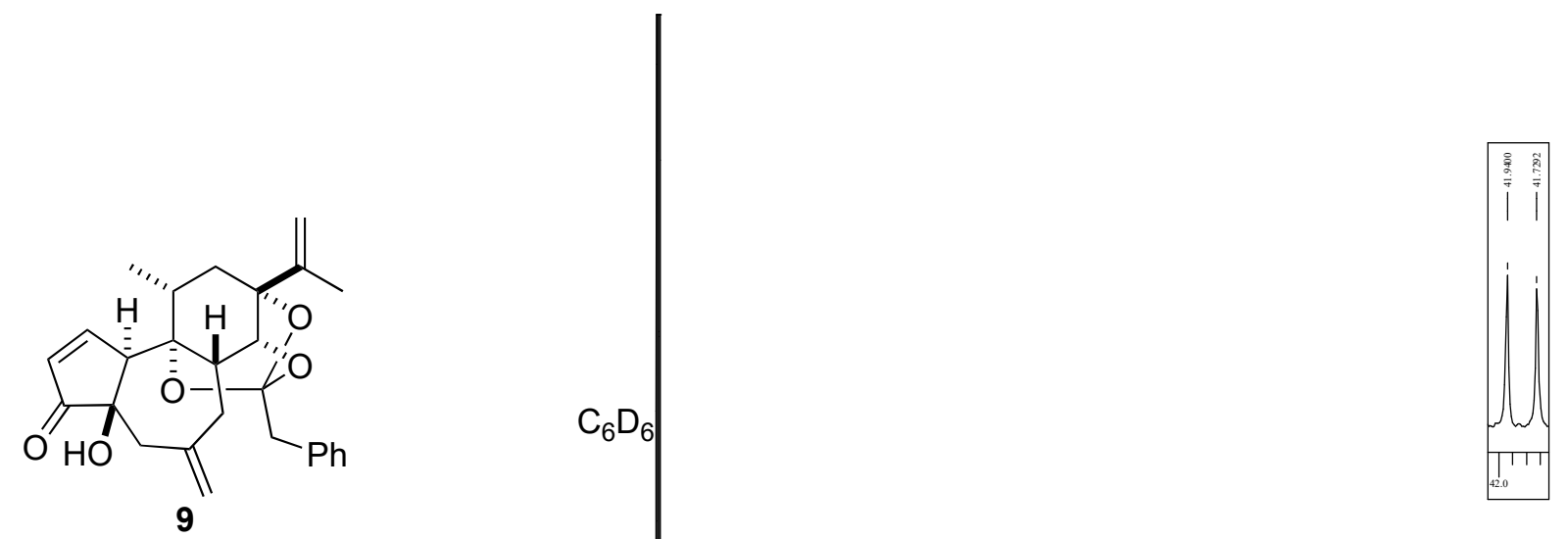

${ }^{13} \mathrm{C}\left\{{ }^{1} \mathrm{H}\right\} \mathrm{NMR}\left(100 \mathrm{MHz}, \mathrm{C}_{6} \mathrm{D}_{6}\right)$

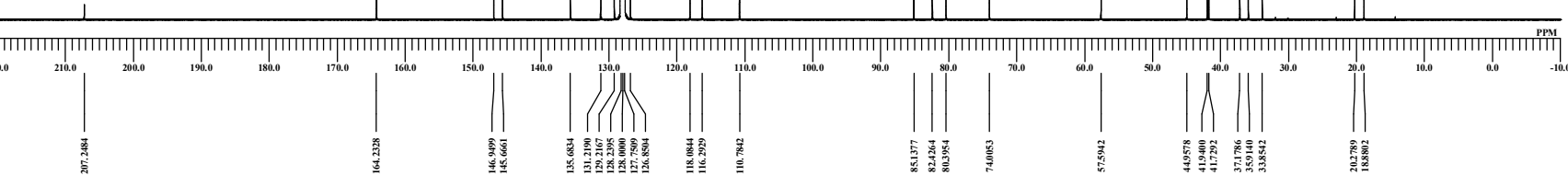



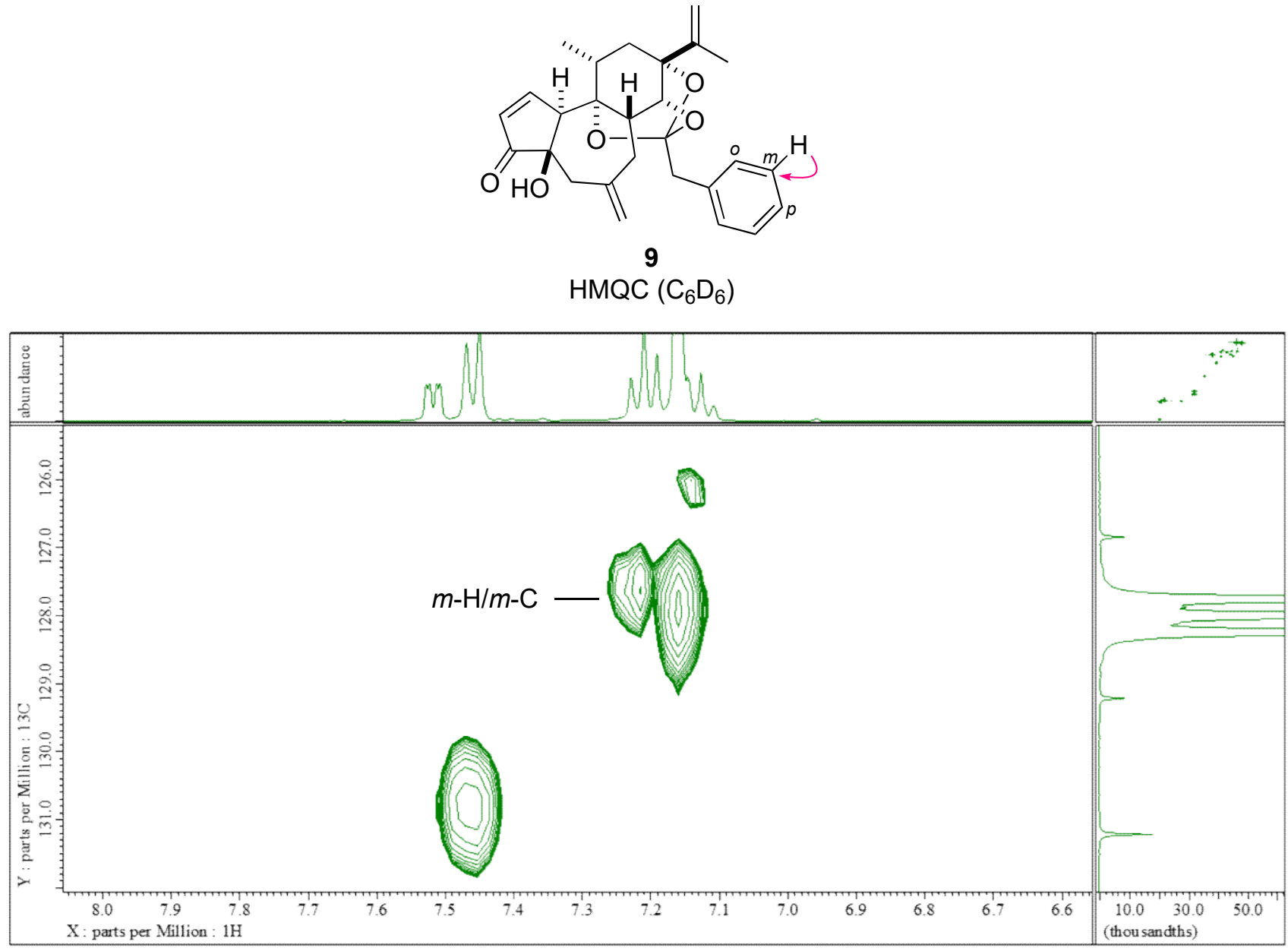


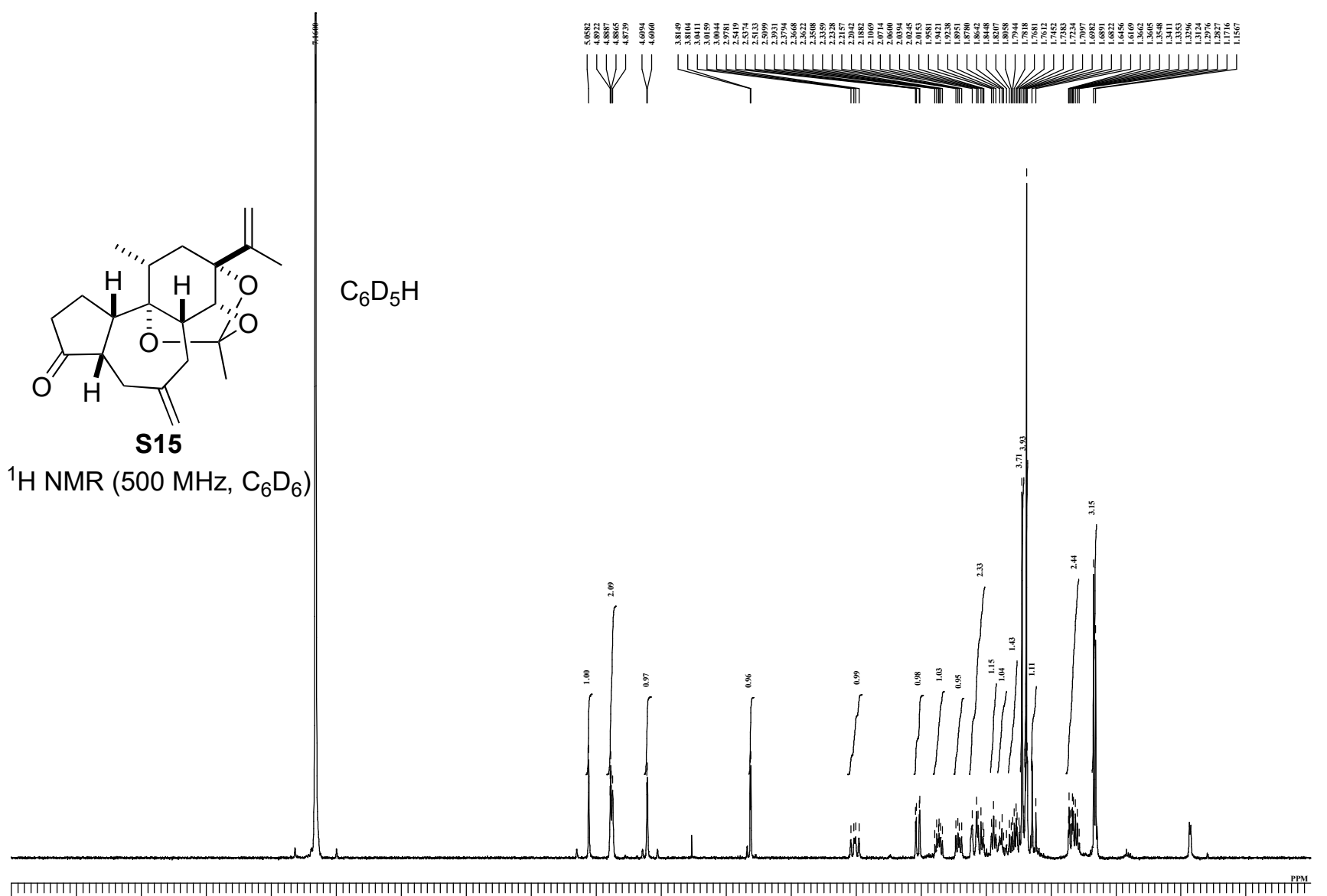

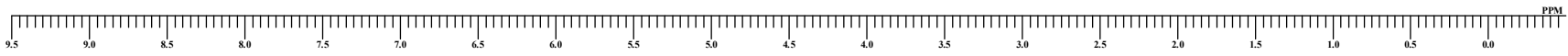

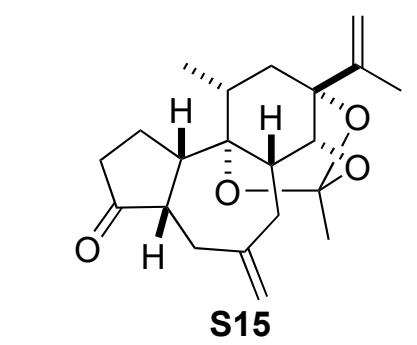

${ }^{13} \mathrm{C}\left\{{ }^{1} \mathrm{H}\right\}$ NMR $\left(100 \mathrm{MHz}, \mathrm{C}_{6} \mathrm{D}_{6}\right)$
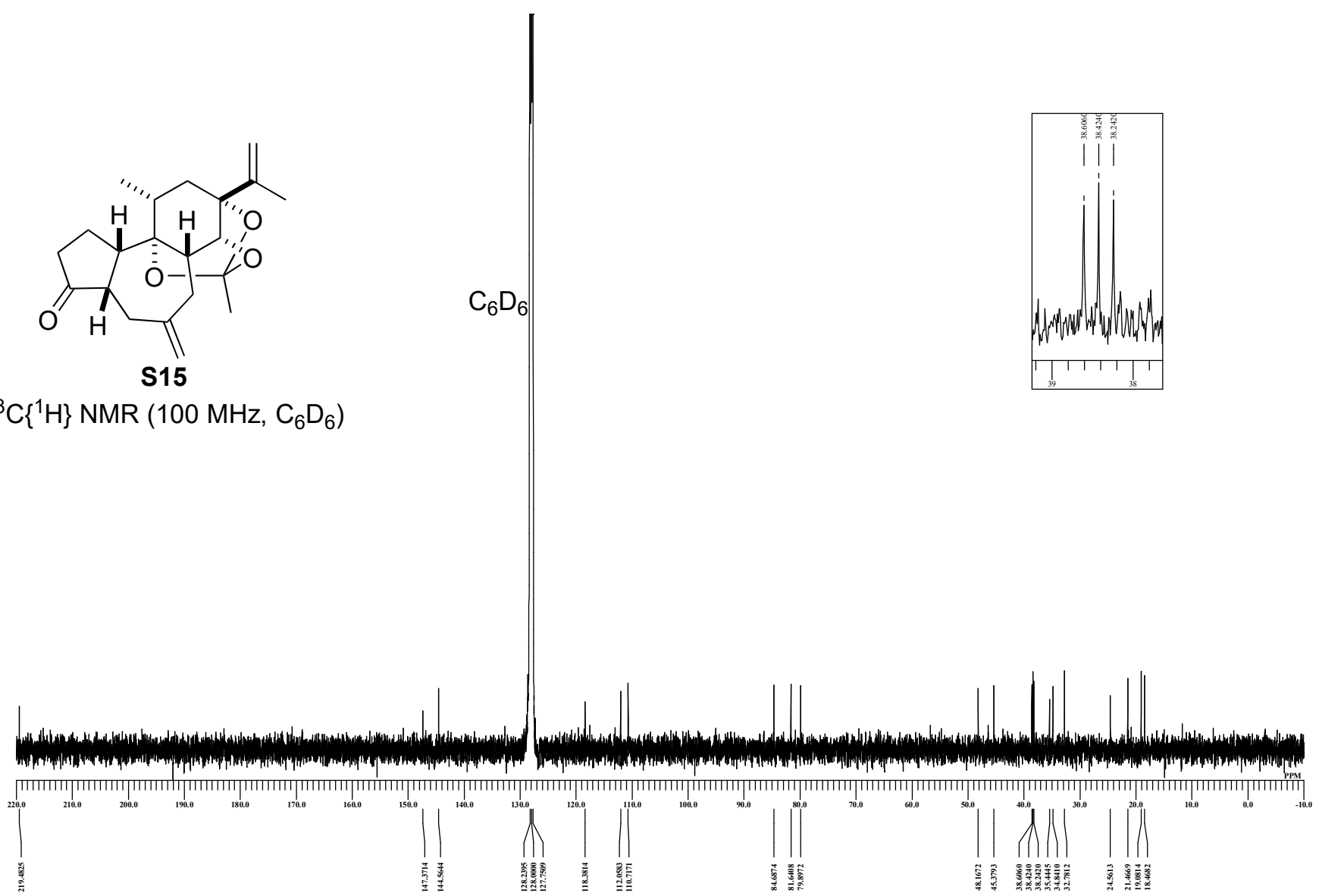

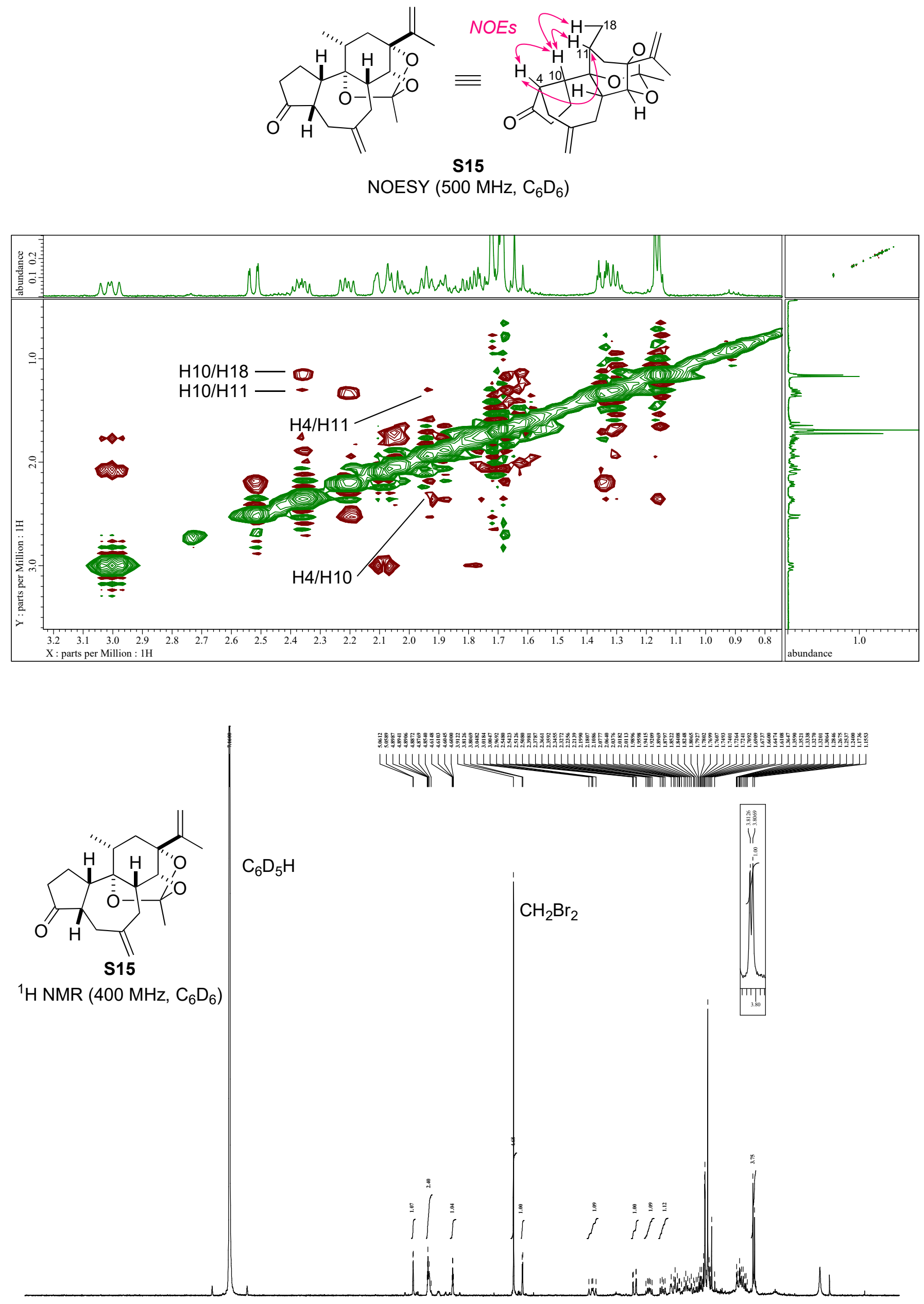

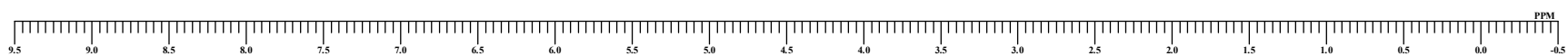

